UNIVERSITY OF OKLAHOMA

GRADUATE COLLEGE

\title{
A REDUCIBILITY PROBLEM FOR EVEN UNITARY GROUPS: THE DEPTH ZERO CASE
}

\author{
A DISSERTATION \\ SUBMITTED TO THE GRADUATE FACULTY \\ in partial fulfillment of the requirements for the \\ Degree of \\ DOCTOR OF PHILOSOPHY
}

By

SUBHA SANDEEP REPAKA

Norman, Oklahoma

2019 


\section{A REDUCIBILITY PROBLEM FOR EVEN UNITARY GROUPS: THE DEPTH ZERO CASE}

\section{A DISSERTATION APPROVED FOR THE DEPARTMENT OF MATHEMATICS}

BY

Dr. Alan Roche, Chair

Dr. S. Lakshmivarahan

Dr. Lucy Lifschitz

Dr. Ameya Pitale

Dr. Tomasz Przebinda 
(c) Copyright by SUBHA SANDEEP REPAKA 2019

All rights reserved. 


\section{Acknowledgements}

First and foremost, I would like to express my deepest gratitude to my advisor Dr. Alan Roche, for his encouragement and help, without which this dissertation would never have been completed. I am forever grateful to him for having me as his student and for inspiring me with his knowledge and expertise in mathematics.

I would also like to thank Dr. S. Lakshmivarahan, Dr. Lucy Lifschitz, Dr. Ameya Pitale and Dr. Tomasz Przebinda for serving in my doctoral committee.

I would also like to thank mathematics department staff for taking care of all the formalities and making my stay here a very enjoyable one. Lastly, I would like to thank my parents and my wife Rama Mounika for their constant encouragement and support. 


\section{Contents}

1 Background 1

1.1 Algebraic groups . . . . . . . . . . . . . . . . . . . 1

1.2 Valuations and local fields . . . . . . . . . . . . . . . . 1

1.3 Representations of locally profinite groups . . . . . . . . . . . . 3

1.3.1 Smooth representations .............. 3

1.3.2 Restriction and induction of representations . . . . . . 5

1.3.3 Supercuspidal representations . . . . . . . . . . 6

1.3.4 Frobenius reciprocity and Mackey's irreducibility criterion 6

1.3.5 Cuspidal representations . . . . . . . . . . . . . . 7

1.4 Unramified characters . . . . . . . . . . . . . . . 7

1.5 Bernstein decomposition ............... 8

1.6 Types and covers ....................... 9

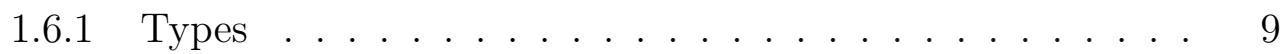

1.6.2 Hecke algebras . . . . . . . . . . . . . . . . 11

1.6.3 Covers ....................... 11

2 Unitary groups $\quad 15$

2.1 Setup . . . . . . . . . . . . . . . . . . 15

2.2 Depth zero representations . . . . . . . . . . . . . . 16 
2.3 Question ............................ 17

2.4 Depth zero supercuspidal representations . . . . . . . . . . . . . . 17

2.5 Siegel parahoric subgroup . . . . . . . . . . . . . . . . . . 19

2.6 Representation of Siegel parahoric subgroup . . . . . . . . . . . . 19

3 Structure of $\mathcal{H}\left(L, \rho_{0}\right)$ and $\mathcal{H}(G, \rho) \quad 22$

3.1 Calculation of $N_{G}\left(\mathfrak{P}_{0}\right) \ldots \ldots \ldots \ldots 22$

$3.2 N_{G}\left(\rho_{0}\right)$ : unramified case . . . . . . . . . . . . . 28

$3.3 N_{G}\left(\rho_{0}\right)$ : ramified case . . . . . . . . . . . . . . 32

3.4 Structure of $\mathcal{H}(G, \rho)$ : unramified case . . . . . . . . . . . . . . . . 37

3.5 Structure of $\mathcal{H}(G, \rho)$ : ramified case . . . . . . . . . . . . . . . . . 46

3.6 Structure of $\mathcal{H}\left(L, \rho_{0}\right) \ldots \ldots \ldots \ldots$

3.7 Calculation of simple $\mathcal{H}\left(L, \rho_{0}\right)$-modules . . . . . . . . . . . . . 55

$4 \quad$ Final computations to answer the question $\quad 59$

4.1 Calculation of $\delta_{P}(\zeta) \ldots \ldots \ldots \ldots \ldots$

4.1.1 Calculation of $\delta_{P}(\zeta)$ : unramified case . . . . . . . . 60

4.1.2 Calculation of $\delta_{P}(\zeta)$ : ramified case . . . . . . . . 60

4.2 Understanding the map $T_{P} \ldots \ldots \ldots$. . . . . . . . . 61

4.2.1 Calculation of $\left(\phi_{0} * \phi_{1}\right)(\zeta) \ldots \ldots 66$

4.2.2 Relation between $g_{0}, g_{1}$ and $T_{P}(\alpha)$ : unramified case . . . 69

4.2.3 Relation between $g_{0}, g_{1}$ and $T_{p}(\alpha)$ : ramified case . . . . . 73

4.3 Calculation of $m_{L}(\pi \nu) \quad \ldots \ldots \ldots$. . . . . . . . . . . . . . 78

4.4 Answering the question . . . . . . . . . . . . . . 80

$\begin{array}{llr}5 & \text { Bibliography } & 83\end{array}$ 


\section{Abstract}

We study a problem concerning parabolic induction in certain p-adic unitary groups. More precisely, for $E / F$ a quadratic extension of p-adic fields the associated unitary group $\mathrm{U}(n, n)$ contains a parabolic subgroup $P$ with Levi component $L$ isomorphic to $\mathrm{GL}_{n}(E)$. Let $\pi$ be an irreducible supercuspidal representation of $L$ of depth zero. We use Hecke algebra methods to determine when the parabolically induced representation $\iota_{P}^{G} \pi$ is reducible. 


\section{Chapter 1}

\section{Background}

In this chapter, we recall the basic definitions and theorems which we need throughout this report.

\section{$1.1 \quad$ Algebraic groups}

Let $F$ be an algebraically closed field. An algebraic group is an algebraic variety over $F$ that is a group such that the multiplication and taking inverse are morphisms of varieties. When the variety is affine we call the group an affine algebraic group. It is well known that every affine algebraic group is isomorphic to some closed subgroup (w.r.t the Zariski topology) of $\mathrm{GL}_{n}(F)$ for some natural number $n$.

\subsection{Valuations and local fields}

Let $F$ be a field. An absolute value on $F$ is a map $||:. F \rightarrow \mathbb{R}_{\geqslant 0}$ such that for any $x, y \in F$, 


$$
\begin{gathered}
|x|=0 \Longleftrightarrow x=0, \\
|x y|=|x||y|, \\
|x+y| \leqslant|x|+|y| .
\end{gathered}
$$

We say $F$ is non-Archimedean if

$$
|x+y| \leqslant \max \{|x|,|y|\} \text { for all } x, y \in F \text {. }
$$

The absolute value |.| defines a topology on $F$ which has as a basis for the open sets, all $U(a, \epsilon)=\{b \in F|| a-b \mid<\epsilon\}, a \in F, \epsilon>0$. We call $F$ a nonArchimedean local field if it is locally compact and complete with respect to a non-trivial non-Archimedean absolute value. Let

$$
\mathfrak{O}_{F}=\{a \in F|| a \mid \leqslant 1\}
$$

which is called the the ring of integers of $F$. Then $\mathfrak{O}_{F}$ is a principal ideal domain with unique maximal ideal

$$
\mathbf{p}_{F}=\{a \in F|| a \mid<1\}
$$

Let $\varpi_{F}$ be a generator of the ideal $\mathbf{p}_{F}$ called a uniformizer of $F$. We denote $\mathfrak{O}_{F} / \mathbf{p}_{F}$ by $k_{F}$ which is a finite field. We call $k_{F}$ the residue field of $F$. We write $\left|k_{F}\right|=q=p^{r}$ for some prime $p$ and some integer $r \geqslant 1$.

Every element in $x \in F^{\times}$can be written uniquely as $x=u \varpi_{F}^{n}$, for some unit $u \in \mathfrak{O}_{F}^{\times}$and $n \in \mathbb{Z}$. We use the notation $n=\nu_{F}(x)$. In these terms the absolute value on $F$ can be given by $|x|=q^{-\nu(x)}=q^{-n}$ for $x \neq 0$ and $|0|=0$.

The ideals

$$
\mathbf{p}_{F}^{n}=\varpi^{n} \mathfrak{O}_{F}=\left\{x \in F|| x \mid \leqslant q^{-n}\right\}, n \in \mathbb{Z}
$$


in $\mathfrak{O}_{F}$ are called the fractional ideals. They are open subgroups of $F$ and give a fundamental system of open neighborhoods of 0 in $F$.

\subsection{Representations of locally profinite groups}

\subsubsection{Smooth representations}

Let $G$ be a topological group. We say $G$ is locally profinite if it is Hausdorff topological space and every open neighborhood of the identity element in $G$ contains a compact open subgroup of $G$.

Let $V$ be a vector space over $\mathbb{C}$ which is not necessarily finite dimensional and $G L(V)$ be the set of all invertible linear operators on $V$. A representation $(\pi, V)$ of $G$ is a homomorphism $\pi$ of groups from $G$ to $G L(V)$.

Suppose $W$ is a subspace of $V$ which is $G$-invariant, i.e., $\pi(g) w \in W$ for all $g \in G, w \in W$. Then restricting the operators $\pi(g)$ to $W$ gives a representation of $G$ in $W$. We call the invariant subspace $W$ a sub-representation of $V$.

If $W^{\prime} \subset W$ are sub-representations of $\pi$, then each $\left.\pi\right|_{W}(g), g \in G$ induces an invertible linear operator $\left.\pi\right|_{W / W^{\prime}}(g)$ on the quotient space $W / W^{\prime}$, and we have $\left(\left.\pi\right|_{W / W^{\prime}}, W / W^{\prime}\right)$ is a representation of $G$ called a sub-quotient of $\pi$. In the special case when $W=V$ we say the representation is a quotient of $\pi$.

A representation $(\pi, V)$ of $G$ is irreducible if the only $G$-invariant subspaces of $V$ are $\{0\}$ and $V$. If $\pi$ is not irreducible then $\pi$ is reducible.

A representation $(\pi, V)$ of $G$ has a finite composition series if there exist $G$ invariant subspaces $V_{j}$ of $V$ such that

$$
0=V_{0} \subsetneq V_{1} \subsetneq V_{2} \cdots \subsetneq V_{r}=V
$$


where each sub-quotient $\left.\pi\right|_{V_{j+1} / V_{j}}, 0 \leqslant j \leqslant r-1$ is irreducible. The sub-quotients $\left.\pi\right|_{V_{j+1} / V_{j}}$ are called the composition factors of $\pi$.

The representation $\pi$ is smooth if for every $v \in V$, there exists a compact open subgroup $K$ of $G$ such that $\pi(k)(v)=v$ for all $k \in K$.

A representation $\pi$ of $V$ is admissible if $V^{K}=\{v \in V \mid \pi(k) v=v\}$ is a finite dimensional subspace of $V$ for every compact open subgroup $K$ of $G$.

Given two representations $\left(\pi_{1}, V_{1}\right)$ and $\left(\pi_{2}, V_{2}\right)$ of $\mathrm{G}$, a linear map $T$ from $V_{1}$ to $V_{2}$ is called an intertwining map if $\pi_{2}(g) \circ T=T \circ \pi_{1}(g)$ for all $g \in G$. We call $\left(\pi_{1}, V_{1}\right)$ and $\left(\pi_{2}, V_{2}\right)$ isomorphic or equivalent representations if there exits an intertwiner $T$ which is an isomorphism. We denote $\operatorname{Hom}_{G}\left(V_{1}, V_{2}\right)$ or $\operatorname{Hom}_{G}\left(\pi_{1}, \pi_{2}\right)$ for the collection of intertwining maps between $V_{1}$ and $V_{2}$. If $\pi_{1}$ and $\pi_{2}$ are representations of the same vector space $V$ and if they are equivalent, then we denote equivalence of representations by $\pi_{1} \simeq \pi_{2}$.

Let $V^{*}=\operatorname{Hom}_{\mathbb{C}}(V, \mathbb{C})$ be the dual space of $V$. Define a dual representation $\left(\pi^{*}, V^{*}\right)$ of $G$ by

$$
\left(\pi^{*}(g) v^{\prime}\right)(v)=v^{\prime}\left(\pi\left(g^{-1}\right) v\right)
$$

for $v \in V, v^{\prime} \in V^{*}$ and $g \in G$. This is a representation of $G$ but it is not necessarily smooth. Therefore we consider the space of all smooth vectors given by $V^{\vee}=\left(V^{*}\right)^{\infty}=\bigcup_{K}\left(V^{*}\right)^{K}$ where the union is taken over all compact open subgroups of $G$. We then define the representation $\left(\pi^{\vee}, V^{\vee}\right)$ as $\pi^{\vee}(g)(v)=\pi^{*}(g)(v)$ for $v \in V^{\vee}, g \in G$. This representation is smooth and we call it the smooth dual or contragradient of $(\pi, V)$.

Given a representation $\pi$ of $H \leqslant G$ and $g \in G$ we let $\pi^{g}$ denote the representation of $H^{g}=g^{-1} H g$ given by $\pi^{g}\left(h^{\prime}\right)=\pi\left(g h^{\prime} g^{-1}\right)$ where $h^{\prime} \in H$ and let ${ }^{g} \pi$ denote the representation of ${ }^{g} H=g H g^{-1}$ given by ${ }^{g} \pi\left(h^{\prime}\right)=\pi\left(g^{-1} h^{\prime} g\right)$ where 
$h^{\prime} \in H$.

\subsubsection{Restriction and induction of representations}

Let $\mathrm{G}$ be a locally profinite group and $(\pi, V)$ be a smooth representation of $G$. Let $H$ be a subgroup of $\mathrm{G}$. The restriction of $\pi$ to $H$ is a representation of $H$ in $V$, denoted by $\left.\pi\right|_{H}$. Now it is natural to ask can we construct a smooth representation of $G$ from smooth representation of $H$ and the answer is yes. The process of constructing a smooth representation of $G$ from smooth representation of $H$ is called smooth induction and the representation of $G$ so obtained is called the smoothly induced representation. We explain the construction below.

Let $(\rho, V)$ be a smooth representation of $H$. The smoothly induced representation is denoted by $\operatorname{Ind}_{H}^{G}(\rho, V)$. Its space is the set of all functions $f: G \rightarrow V$ such that

1. $f(h g)=\rho(h) f(g)$ for $h \in H, g \in G$.

2. There is a compact open subgroup $\mathrm{K}$ of $\mathrm{G}$ such that $f(g k)=f(g)$ for $g \in G, k \in K$.

The action of $G$ is given by $g . f(x)=f(x g)$ where $f \in \operatorname{Ind}_{H}^{G}(\rho, V), x \in G$.

Given a smooth representation $(\rho, V)$ of $H$, we can also define another type of smooth representation of $G$ denoted by $c-\operatorname{In} d_{H}^{G}(\rho, V)$. It consists of all functions in $\operatorname{Ind}_{H}^{G}(\rho, V)$ which are compactly supported modulo $H$. This means if $f \in$ $\operatorname{Ind}_{H}^{G}(\rho, V)$ is such that support of $f$ is compact in $G / H$ then $f \in c-\operatorname{Ind}_{H}^{G}(\rho, V)$. The action of $G$ on $c$ - $I n d_{H}^{G}(\rho, V)$ is again given by $g \cdot f(x)=f(x g)$ where $f \in c$ $\operatorname{Ind}_{H}^{G}(\rho, V), x \in G$.

Now there is another notion called normalized induction. Let $G$ be the group of $F$-points of a reductive algebraic group defined over a non-Archimedean local 
field $F$. Let $P$ be a parabolic subgroup of $G$. Write $P=L \ltimes U$ where $L$ is the Levi component of $P$ and $U$ is the unipotent radical of $P$. Let $(\rho, V)$ be a smooth representation of $P$. The normalized induction $\iota_{P}^{G}(\rho, V)$ is defined as $\iota_{P}^{G}(\rho, V)=$

$\operatorname{Ind}_{P}^{G}\left(\rho \otimes \delta_{P}^{1 / 2}\right), \delta_{P}$ is a character of $P$ defined as $\delta_{P}(p)=\left\|\left.\operatorname{det}(\operatorname{Ad} p)\right|_{\operatorname{Lie} U}\right\|_{F}$ for $p \in P$ and Lie $U$ is the Lie-algebra of $U$. We shall use $\iota_{P}^{G}(\rho)$ for $\iota_{P}^{G}(\rho, V)$ in this report. We work with normalized induced representations rather than induced representations in this report as results look more appealing.

\subsubsection{Supercuspidal representations}

Let $G$ be the group of $F$-points of a reductive algebraic group defined over a non-Archimedean local field $F$. A representation $(\pi, V)$ of $G$ is supercuspidal if

$$
\operatorname{Hom}_{G}\left(\pi, \operatorname{Ind} d_{P}^{G} \tau\right)=\{0\}
$$

for any proper parabolic subgroups $P$ of $G$ and any representation $\tau$ of a Levi component of $P$.

\subsubsection{Frobenius reciprocity and Mackey's irreducibility criterion}

We recall Frobenius reciprocity. Let $G$ be a locally profinite group and $(\pi, V)$ be a representation of $G$. Let $H$ be an open subgroup of $G$ and $(\rho, W)$ be a representation of $H$. Then

$$
\operatorname{Hom}_{G}\left(c-\operatorname{Ind} d_{H}^{G} \rho, \pi\right) \simeq \operatorname{Hom}_{H}\left(\rho,\left.\pi\right|_{H}\right)
$$


For $H$ a closed subgroup of $G$, we have

$$
\operatorname{Hom}_{G}\left(\pi, \operatorname{Ind}_{H}^{G} \rho\right) \simeq \operatorname{Hom}_{H}\left(\left.\pi\right|_{H}, \rho\right)
$$

We recall Mackey's Irreducibility Criterion. Let $G$ be a locally profinite group and $H$ be an open, compact subgroup of $G$. Let $(\pi, V)$ be a smooth representation of $H$. Then $\operatorname{Ind}_{H}^{G} \pi$ is irreducible exactly when

$$
\operatorname{Hom}\left(\left.\pi^{g}\right|_{H \cap H^{g}},\left.\pi\right|_{H \cap H^{g}}\right)=0
$$

for $g \notin H$.

\subsubsection{Cuspidal representations}

Let $G$ be a finite group of Lie type. Let $(\rho, V)$ be an irreducible representation of $G$. For $P$ a parabolic subgroup of $G$, we write $U_{P}$ for its unipotent radical. We say $(\rho, V)$ is a cuspidal representation of $G \Longleftrightarrow V^{U_{P}}=0$ for all proper parabolic subgroups $P$ of $G$.

\subsection{Unramified characters}

Let $G$ be the group of $F$-points of an algebraic group defined over a non-Archimedean local field $F$. Write $G^{\circ}$ for the smallest subgroup of $G$ containing the compact open subgroups of $G$. We say a character $\nu: G \longrightarrow \mathbb{C}^{\times}$is unramified if $\left.\nu\right|_{G^{\circ}}=1$, i.e., $\nu$ is trivial on $G^{\circ}$. Let the group of unramified characters of $G$ be denoted by $\mathrm{X}_{n r}(G)$. 


\subsection{Bernstein decomposition}

Let $G$ be the $F$-rational points of a reductive algebraic group defined over a non-Archimedean local field $F$. According to Theorem 3.3 in [6], we have the following Propn.

Proposition 1.1. 1. Let $L$ be a Levi subgroup of $G$ (i.e., a Levi component of a parabolic subgroup $P$ of $G)$. Let $\sigma$ be an irreducible smooth supercuspidal representation of $L$. Then $\iota_{P}^{G} \sigma$ has finite length for every parabolic subgroup $P$ with Levi component $L$. Further, the set of the composition factors or irreducible sub-quotients of $\iota_{P}^{G} \sigma$ is independent of $P$.

2. Let $L_{1}, L_{2}$ be Levi subgroups of $G$ and $\sigma_{1}, \sigma_{2}$ be irreducible supercuspidal smooth representations of $L_{1}, L_{2}$ respectively. Then for any parabolic subgroups $P_{1}, P_{2}$ with Levi components $L_{1}, L_{2}$ respectively, we have the representations $\iota_{P_{1}}^{G} \sigma_{1}, \iota_{P_{2}}^{G} \sigma_{2}$ either have the same set of composition factors or have no composition factors in common. Now the representations $\iota_{P_{1}}^{G} \sigma_{1}$ and $\iota_{P_{2}}^{G} \sigma_{2}$ have the same set of composition factors $\Longleftrightarrow$ the pairs $\left(L_{1}, \sigma_{1}\right)$ and $\left(L_{2}, \sigma_{2}\right)$ are conjugate; that is, there is an element $g \in G$ such that $L_{2}=L_{1}^{g}=g^{-1} L_{1} g$ and $\sigma_{2} \simeq \sigma_{1}^{g}$.

3. Let $(\pi, V)$ be an irreducible smooth representation of $G$. Then there exists a parabolic subgroup $P$ of $G$ with Levi component $L$, unipotent radical $U$ and an irreducible supercuspidal smooth representation $\sigma$ of $L$ such that $\pi$ is equivalent to an irreducible sub-quotient or a composition factor of $\iota_{P}^{G} \sigma$. We refer to the pair $(L, \sigma)$ where $L$ is a Levi subgroup of $G$ and $\sigma$ is an irreducible supercuspidal smooth representation of $L$ as a cuspidal pair.

Now by Propn. 1.1, there exists unique conjugacy class of cuspidal pairs $(L, \sigma)$ 
with the property that $\pi$ is isomorphic to a composition factor of $\iota_{P}^{G} \sigma$ for some parabolic subgroup $P$ of $G$. We call this conjugacy class of cuspidal pairs, the cuspidal support of $(\pi, V)$.

Given two cuspidal supports $\left(L_{1}, \sigma_{1}\right)$ and $\left(L_{2}, \sigma_{2}\right)$ of $(\pi, V)$, we say they are inertially equivalent if there exists $g \in G$ and $\chi \in \mathrm{X}_{n r}\left(L_{2}\right)$ such that $L_{2}=L_{1}^{g}$ and $\sigma_{1}^{g} \simeq \sigma_{2} \otimes \chi$. We write $[L, \sigma]_{G}$ for the inertial equivalence class or inertial support of $(\pi, V)$. Let $\mathfrak{B}(G)$ denote the set of inertial equivalence classes $[L, \sigma]_{G}$.

Let $\mathfrak{R}(G)$ denote the category of smooth representations of $G$. Let $\mathfrak{R}^{s}(G)$ be the full sub-category of smooth representations of $G$ with the property that $(\pi, V) \in o b\left(\Re^{s}(G)\right) \Longleftrightarrow$ every irreducible sub-quotient of $\pi$ has inertial support $s=[L, \sigma]_{G}$.

We can now state the Bernstein decomposition:

$$
\mathfrak{R}(G)=\prod_{s \in \mathfrak{B}(G)} \mathfrak{R}^{s}(G) .
$$

\subsection{Types and covers}

Let $G$ be the $F$-rational points of a reductive algebraic group defined over a non-Archimedean local field $F$.

\subsubsection{Types}

Let $K$ be a compact open subgroup of $G$. Let $(\rho, W)$ be an irreducible smooth representation of $K$ and $(\pi, V)$ be a smooth representation of $G$. Let $V^{\rho}$ be the $\rho$-isotopic subspace of $V$. Thus $V^{\rho}$ is the sum of all irreducible $K$-subspaces of $V$ which are equivalent to $\rho$. 


$$
V^{\rho}=\sum_{W^{\prime}} W^{\prime}
$$

where the sum is over all $W^{\prime}$ such that $\left(\left.\pi\right|_{K}, W^{\prime}\right) \simeq(\rho, W)$.

Let $\mathcal{H}(G)$ be the space of all locally constant compactly supported functions $f: G \rightarrow \mathbb{C}$. This is a $\mathbb{C}$ - algebra under convolution $*$. So for elements $f, g \in \mathcal{H}(G)$ we have

$$
(f * g)(x)=\int_{G} f(y) g\left(y^{-1} x\right) d \mu(y)
$$

Here we have fixed a Haar measure $\mu$ on $G$. Let $(\pi, V)$ be a representation of $G$. Then $\mathcal{H}(G)$ acts on $V$ via

$$
h v=\int_{G} h(x) \pi(x) v d \mu(x)
$$

for $h \in \mathcal{H}(G), v \in V$. Let $e_{\rho}$ be the element in $\mathcal{H}(G)$ with support $K$ such that

$$
e_{\rho}(x)=\frac{\operatorname{dim} \rho}{\mu(K)} \operatorname{tr}_{W}\left(\rho\left(x^{-1}\right)\right), x \in K \text {. }
$$

We have $e_{\rho} * e_{\rho}=e_{\rho}$ and $e_{\rho} V=V^{\rho}$ for any smooth representation $(\pi, V)$ of $G$.

Let $\mathfrak{R}_{\rho}(G)$ be the full sub-category of $\mathfrak{R}(G)$ consisting of all $(\pi, V)$ where $V$ is generated by $V^{\rho}$. So $(\pi, V) \in \mathfrak{R}_{\rho}(G)$ if and only if $V=\mathcal{H}(G) * e_{\rho} V$. We now state the definition of a type.

Definition 1.2. Let $s \in \mathfrak{B}(G)$. We say that $(K, \rho)$ is an $s$-type in $G$ if $\mathfrak{R}_{\rho}(G)=$ $\mathfrak{R}^{s}(G)$. 


\subsubsection{Hecke algebras}

Let $K$ be a compact open subgroup of $G$. Let $(\rho, W)$ be an irreducible smooth representation of $K$. Here we introduce the Hecke algebra $\mathcal{H}(G, \rho)$.

$$
\mathcal{H}(G, \rho)=\left\{\begin{array}{l|l}
f: G \rightarrow \operatorname{End}_{\mathbb{C}}\left(\rho^{\vee}\right) & \begin{array}{l}
\operatorname{supp}(f) \text { is compact and } \\
f\left(k_{1} g k_{2}\right)=\rho^{\vee}\left(k_{1}\right) f(g) \rho^{\vee}\left(k_{2}\right) \\
\text { where } k_{1}, k_{2} \in K, g \in G
\end{array}
\end{array}\right\}
$$

Then $\mathcal{H}(G, \rho)$ is a $\mathbb{C}$-algebra with multiplication given by convolution $*$ w.r.t some fixed Haar measure $\mu$ on $G$. So for elements $f, g \in \mathcal{H}(G)$ we have

$$
(f * g)(x)=\int_{G} f(y) g\left(y^{-1} x\right) d \mu(y) .
$$

The importance of types is seen from the following result. Let $\pi$ be a smooth representation in $\mathfrak{R}^{s}(G)$. Let $\mathcal{H}(G, \rho)-\operatorname{Mod}$ denote the category of $\mathcal{H}(G, \rho)$ modules. If $(K, \rho)$ is an $s$-type then $m_{G}: \mathfrak{R}^{s}(G) \longrightarrow \mathcal{H}(G, \rho)-\operatorname{Mod}$ given by $m_{G}(\pi)=\operatorname{Hom}_{K}(\rho, \pi)$ is an equivalence of categories.

\subsubsection{Covers}

Let $K$ be a compact open subgroup of $G$. Let $P=L \ltimes U$ be a parabolic subgroup of $G$. The notation means that $P$ has unipotent radical $U$ and that $L$ is a Levi component of $P$. Let $\bar{P}=L \ltimes \bar{U}$ be the $L$-opposite of $P$. Thus $P \cap \bar{P}=L$. Let $(\rho, W)$ be an irreducible representation of $K$. Then we say $(K, \rho)$ is decomposed with respect to $(L, P)$ if the following hold:

1. $K=(K \cap \bar{U})(K \cap L)(K \cap U)$. 
2. $(K \cap \bar{U}),(K \cap U) \leqslant \operatorname{ker} \rho$.

Suppose $(K, \rho)$ is decomposed with respect to $(L, P)$. We set $K_{L}=K \cap L$ and $\rho_{L}=\left.\rho\right|_{K_{L}}$. We say an element $g \in G$ intertwines $\rho$ if $\operatorname{Hom}_{K^{g} \cap K}\left(\rho^{g}, \rho\right) \neq 0$. Let $\mathfrak{I}_{G}(\rho)=\{x \in G \mid x$ intertwines $\rho\}$. We have the Hecke algebras $\mathcal{H}(G, \rho)$ and $\mathcal{H}\left(L, \rho_{L}\right)$. We write

$$
\mathcal{H}(G, \rho)_{L}=\{f \in \mathcal{H}(G, \rho) \mid \operatorname{supp}(f) \subseteq K L K\} .
$$

We recall some results and constructions from pages 606-612 in [2]. These allow us to transfer questions about parabolic induction into questions concerning the module theory of appropriate Hecke algebras.

Proposition 1.3. Let $(K, \rho)$ decompose with respect to $(L, P)$. Then

1. $\rho_{L}$ is irreducible.

2. $\mathfrak{I}_{L}\left(\rho_{L}\right)=\mathfrak{I}_{G}(\rho) \cap L$.

3. There is an embedding $T: \mathcal{H}\left(L, \rho_{L}\right) \longrightarrow \mathcal{H}(G, \rho)$ such that if $f \in \mathcal{H}\left(L, \rho_{L}\right)$ has support $K_{L} z K_{L}$ for some $z \in L$, then $T(f)$ has support $K z K$.

4. The map $T$ induces an isomorphism of vector spaces:

$$
\mathcal{H}\left(L, \rho_{L}\right) \stackrel{\simeq}{\rightarrow} \mathcal{H}(G, \rho)_{L} .
$$

Definition 1.4. An element $z \in L$ is called $(K, P)$-positive element if:

1. $z(K \cap \bar{U}) z^{-1} \subseteq K \cap \bar{U}$.

2. $z^{-1}(K \cap U) z \subseteq K \cap U$. 
Definition 1.5. An element $z \in L$ is called strongly $(K, P)$-positive element if:

1. $z$ is $(K, P)$ positive.

2. $z$ lies in center of $L$.

3. For and compact open subgroups $K$ and $K^{\prime}$ of $U$ there exists $m \geqslant 0$ and $m \in \mathbb{Z}$ such that $z^{m} K z^{-m} \subseteq K^{\prime}$.

4. For and compact open subgroups $K$ and $K^{\prime}$ of $U$ there exists $m \geqslant 0$ and $m \in \mathbb{Z}$ such that $z^{-m} K z \subseteq K^{\prime}$.

Proposition 1.6. Strongly $(K, P)$-positive elements exist and given a strongly positive element $z \in L$, there exists a unique function $\phi_{z} \in \mathcal{H}\left(L, \rho_{L}\right)$ with support $K_{L} z K_{L}$ such that $\phi_{z}(z)$ is identity function in $\operatorname{End}_{\mathbb{C}}\left(\rho_{L}\right)$.

$$
\mathcal{H}^{+}\left(L, \rho_{L}\right)=\left\{\begin{array}{l|l}
f: G \rightarrow \operatorname{End}_{\mathbb{C}}\left(\rho_{L}^{\vee}\right) & \begin{array}{l}
\operatorname{supp}(f) \text { is compact and consists } \\
\text { of strongly }(K, P) \text {-positive elements } \\
\text { and } f\left(k_{1} l k_{2}\right)=\rho_{L}^{\vee}\left(k_{1}\right) f(l) \rho_{L}^{\vee}\left(k_{2}\right) \\
\text { where } k_{1}, k_{2} \in K_{L}, l \in L
\end{array}
\end{array}\right\} .
$$

The isomorphism of vector spaces $T: \mathcal{H}\left(L, \rho_{L}\right) \longrightarrow \mathcal{H}(G, \rho)_{L}$ restricts to an embedding of algebras:

$$
T^{+}: \mathcal{H}^{+}\left(L, \rho_{L}\right) \longrightarrow \mathcal{H}(G, \rho)_{L} \hookrightarrow \mathcal{H}(G, \rho) .
$$

Proposition 1.7. The embedding $T^{+}$extends to an embedding of algebras $t: \mathcal{H}\left(L, \rho_{L}\right) \longrightarrow \mathcal{H}(G, \rho)$ if and only if $T^{+}\left(\phi_{z}\right)$ is invertible for some strongly $(K, P)$-positive element $z$, where $\phi_{z} \in \mathcal{H}\left(L, \rho_{L}\right)$ has support $K_{L} z K_{L}$ with $\phi_{z}(z)=$ 1. 
Definition 1.8. Let $L$ be a proper Levi subgroup of $G$. Let $K_{L}$ be a compact open subgroup of $L$ and $\rho_{L}$ be an irreducible smooth representation of $K_{L}$. Let $K$ be a compact open subgroup of $G$ and $\rho$ be an irreducible, smooth representation of $K$. Then we say $(K, \rho)$ is a $G$-cover of $\left(K_{L}, \rho_{L}\right)$ if

1. The pair $(K, \rho)$ is decomposed with respect to $(L, P)$ for every parabolic subgroup $P$ of $G$ with Levi component $L$.

2. $K \cap L=K_{L}$ and $\left.\rho\right|_{L} \simeq \rho_{L}$.

3. The embedding $T^{+}: \mathcal{H}^{+}\left(L, \rho_{L}\right) \longrightarrow \mathcal{H}(G, \rho)$ extends to an embedding of algebras $t: \mathcal{H}\left(L, \rho_{L}\right) \longrightarrow \mathcal{H}(G, \rho)$.

Proposition 1.9. Let $s_{L}=[L, \pi]_{L} \in \mathfrak{B}(L)$ and $s=[L, \pi]_{G} \in \mathfrak{B}(G)$. Say $\left(K_{L}, \rho_{L}\right)$ is an $s_{L}$-type and $(K, \rho)$ is a $G$-cover of $\left(K_{L}, s_{L}\right)$. Then $(K, \rho)$ is an s-type.

Recall the categories $\mathfrak{R}^{s_{L}}(L), \mathfrak{R}^{s}(G)$ where $s_{L}=[L, \pi]_{L}$ and $s=[L, \pi]_{G}$. Also recall $\mathcal{H}(G, \rho)-\operatorname{Mod}$ is the category of $\mathcal{H}(G, \rho)$-modules. Let $\mathcal{H}\left(L, \rho_{L}\right)-\operatorname{Mod}$ be the category of $\mathcal{H}\left(L, \rho_{L}\right)$-modules. The functors $\iota_{P}^{G}, m_{G}$ were defined earlier. Let $\pi \in \mathfrak{R}^{s_{L}}(L)$. Then the functor $m_{L}: \mathfrak{R}^{s_{L}}(L) \longrightarrow \mathcal{H}\left(L, \rho_{L}\right)-M o d$ is given by $m_{L}(\pi)=\operatorname{Hom}_{K_{L}}\left(\rho_{L}, \pi\right)$. The functor $\left(T_{P}\right)_{*}: \mathcal{H}\left(L, \rho_{L}\right)-\operatorname{Mod} \longrightarrow \mathcal{H}(G, \rho)-M o d$ is defined later in this report.

The importance of covers is seen from the following commutative diagram which we will use in answering the question which we pose later in this report.

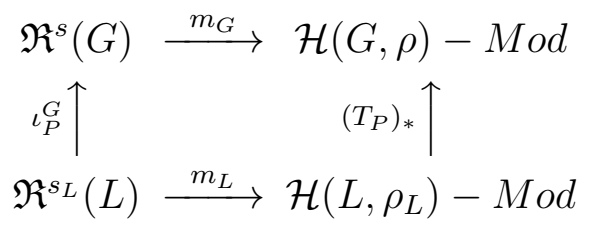




\section{Chapter 2}

\section{Unitary groups}

\section{$2.1 \quad$ Setup}

Let $E / F$ be a quadratic Galois extension of non-Archimedean local fields where char $F \neq 2$. Write - for the non-trivial element of $\operatorname{Gal}(E / F)$. The group $\mathrm{U}(n, n)$ is given by

$$
\mathrm{U}(n, n)=\left\{g \in \mathrm{GL}_{2 n}(E) \mid{ }^{t} \bar{g} J g=J\right\}
$$

for $J=\left[\begin{array}{ll}0 & 1 \\ 1 & 0\end{array}\right]$ where each block is of size $n$ and for $g=\left(g_{i j}\right)$ we write $\bar{g}=\left(\bar{g}_{i j}\right)$. We write $\mathfrak{O}_{E}$ and $\mathfrak{O}_{F}$ for the ring of integers in $E$ and $F$ respectively. Similarly, $\mathbf{p}_{E}$ and $\mathbf{p}_{F}$ denote the maximal ideals in $\mathfrak{O}_{E}$ and $\mathfrak{O}_{F}$ and $k_{E}=\mathfrak{O}_{E} / \mathbf{p}_{E}$ and $k_{F}=\mathfrak{O}_{F} / \mathbf{p}_{F}$ denote the residue class fields of $\mathfrak{O}_{E}$ and $\mathfrak{O}_{F}$.

There are two kinds of extensions of $E$ over $F$. One is the unramified extension and the other one is the ramified extension. In the unramified case, we can choose uniformizers $\varpi_{E}, \varpi_{F}$ in $E, F$ such that $\varpi_{E}=\varpi_{F}$ so that we have $\left[k_{E}: k_{F}\right]=$ $2, \operatorname{Gal}\left(k_{E} / k_{F}\right) \cong \operatorname{Gal}(E / F)$. As $\varpi_{E}=\varpi_{F}$, so $\bar{\varpi}_{E}=\varpi_{E}$ since $\varpi_{F} \in F$. As 
$k_{F}=\mathbb{F}_{q}$, so $k_{E}=\mathbb{F}_{q^{2}}$ in this case. In the ramified case, we can choose uniformizers $\varpi_{E}, \varpi_{F}$ in $E, F$ such that $\varpi_{E}^{2}=\varpi_{F}$ so that we have $\left[k_{E}: k_{F}\right]=1, \operatorname{Gal}\left(k_{E} / k_{F}\right)=$ 1. As $\varpi_{E}^{2}=\varpi_{F}$, we can further choose $\varpi_{E}$ such that $\bar{\varpi}_{E}=-\varpi_{E}$. As $k_{F}=\mathbb{F}_{q}$, so $k_{E}=\mathbb{F}_{q}$ in this case.

We write $P$ for the Siegel parabolic subgroup of $G$. Write $L$ for the Siegel Levi component of $P$ and $U$ for the unipotent radical of $P$. Thus $P=L \ltimes U$ with

$$
L=\left\{\left[\begin{array}{cc}
a & 0 \\
0 & { }^{t} \bar{a}^{-1}
\end{array}\right] \mid a \in \mathrm{GL}_{n}(E)\right\}
$$

and

$$
U=\left\{\left[\begin{array}{cc}
1 & X \\
0 & 1
\end{array}\right] \mid X \in \mathrm{M}_{n}(E), X+{ }^{t} \bar{X}=0\right\} .
$$

Let $K_{0}=\mathrm{GL}_{n}\left(\mathfrak{O}_{E}\right)$ and $K_{1}=1+\varpi_{E} \mathrm{M}_{n}\left(\mathfrak{O}_{E}\right)$. Note $K_{1}=1+\varpi_{E} \mathrm{M}_{n}\left(\mathfrak{O}_{E}\right)$ is the kernel of the surjective group homomorphism

$$
\left(g_{i j}\right) \longrightarrow\left(g_{i j}+\mathbf{p}_{\mathbf{E}}\right): \mathrm{GL}_{n}\left(\mathfrak{O}_{E}\right) \longrightarrow \mathrm{GL}_{n}\left(k_{E}\right)
$$

\subsection{Depth zero representations}

The general definition of depth zero representation is given by Theorem 3.5 in [9]. However, for our specific problem we say $\pi$ is a depth zero representation of Siegel Levi component $L$ of $P$ if $\pi^{K_{1}} \neq 0$. 


\subsection{Question}

Let $\pi$ be an irreducible supercuspidal representation of $L$ of depth zero. We look at the family of representations $\iota_{P}^{G}(\pi \nu)$ for $\nu \in \chi_{n r}(L)$. We want to determine the set of such $\nu$ for which this induced representation is irreducible. By general theory, this is a finite set.

\subsection{Depth zero supercuspidal representations}

Suppose $\tau$ is an irreducible cuspidal representation of $\mathrm{GL}_{n}\left(k_{E}\right)$ inflated to a representation of $\mathrm{GL}_{n}\left(\mathfrak{O}_{E}\right)=K_{0}$. Then let $\widetilde{K_{0}}=Z K_{0}$ where $Z=Z\left(\mathrm{GL}_{n}(E)\right)=$ $\left\{\lambda 1_{n} \mid \lambda \in E^{\times}\right\}$. As any element of $E^{\times}$can be written as $u \varpi_{E}^{n}$ for some $u \in \mathfrak{O}_{E}^{\times}$ and $m \in \mathbb{Z}$. So in fact, $\widetilde{K_{0}}=<\varpi_{E} 1_{n}>K_{0}$.

Let $(\pi, V)$ be a representation of $G$ and $1_{V}$ be the identity linear transformation of $V$. As $\varpi_{E} 1_{n} \in Z$, so $\pi\left(\varpi_{E} 1_{n}\right)=\omega_{\pi}\left(\varpi_{E} 1_{n}\right) 1_{V}$ where $\omega_{\pi}: Z \longrightarrow \mathbb{C}^{\times}$is the central character of $\pi$.

Let $\widetilde{\tau}$ be a representation of $\widetilde{K}_{0}$ such that:

1. $\widetilde{\tau}\left(\varpi_{E} 1_{n}\right)=\omega_{\pi}\left(\varpi_{E} 1_{n}\right) 1_{V}$,

2. $\left.\widetilde{\tau}\right|_{K_{0}}=\tau$.

Say $\omega_{\pi}\left(\varpi_{E} 1_{n}\right)=z$ where $z \in \mathbb{C}^{\times}$. Now call $\widetilde{\tau}=\widetilde{\tau}_{z}$. We have extended $\tau$ to

$\widetilde{\tau}_{z}$ which is a representation of $\widetilde{K}_{0}$, so that $Z$ acts by $\omega_{\pi}$. Hence $\left.\pi\right|_{\widetilde{K}_{0}} \supseteq \widetilde{\tau}_{z}$ which implies that $\operatorname{Hom}_{\widetilde{K}_{0}}\left(\widetilde{\tau}_{z},\left.\pi\right|_{\widetilde{K}_{0}}\right) \neq 0$.

By Frobenius reciprocity for induction from open subgroups,

$$
\operatorname{Hom}_{\widetilde{K}_{0}}\left(\widetilde{\tau}_{z},\left.\pi\right|_{\widetilde{K}_{0}}\right) \simeq \operatorname{Hom}_{G}\left(c-\operatorname{Ind} d_{\widetilde{K}_{0}}^{G} \widetilde{\tau}_{z}, \pi\right)
$$


Thus $c-\operatorname{Hom}_{G}\left(\operatorname{Ind}_{\widetilde{K}_{0}}^{G} \widetilde{\tau}_{z}, \pi\right) \neq 0$. So there exists a non-zero $G$-map from $c$ $\operatorname{Ind} d_{\widetilde{K}_{0}}^{G} \widetilde{\tau}_{z}$ to $\pi$. As $\tau$ is cuspidal representation, using Cartan decompostion and Mackey's criteria we can show that $c-\operatorname{In} d_{\widetilde{K}_{0}}^{G} \widetilde{\tau}_{z}$ is irreducible. So $\pi \simeq c-\operatorname{In} d_{\widetilde{K}_{0}}^{G} \widetilde{\tau}_{z}$. As $c-\operatorname{In} d_{\widetilde{K}_{0}}^{G} \widetilde{\tau}_{z}$ is irreducible supercuspidal representation of $G$ of depth zero, so $\pi$ is irreducible supercuspidal representation of $G$ of depth zero.

Conversely, let $\pi$ is a depth zero representation of $\mathrm{GL}_{n}(E)$. So $\pi^{K_{1}} \neq\{0\}$. Hence $\left.\pi\right|_{K_{1}} \supseteq 1_{K_{1}}$, where $1_{K_{1}}$ is trivial representation of $K_{1}$. This means $\left.\pi\right|_{K_{0}} \supseteq \tau$, where $\tau$ is an irreducible representation of $K_{0}$ such that $\left.\tau\right|_{K_{1}} \supseteq 1_{K_{1}}$. So $\tau$ is trivial on $K_{1}$. So $\left.\pi\right|_{K_{0}}$ contains an irreducible representation $\tau$ of $K_{0}$ such that $\left.\tau\right|_{K_{1}}$ is trivial. So $\tau$ can be viewed as an irreducible representation of $K_{0} / K_{1} \cong \mathrm{GL}_{n}\left(k_{E}\right)$ inflated to $K_{0}=\mathrm{GL}_{n}\left(\mathfrak{O}_{E}\right)$. The representation $\tau$ is cuspidal by (a very special case of) A.1 Appendix [8].

So we have the following bijection of sets:

$$
\begin{aligned}
& \left\{\begin{array}{l}
\text { Isomorphism classes of irreducible } \\
\text { cuspidal representations of } \mathrm{GL}_{n}\left(k_{E}\right)
\end{array}\right\} \times \mathbb{C}^{\times} \longleftrightarrow\left\{\begin{array}{l}
\text { Isomorphism classes } \\
\text { of irreducible } \\
\text { supercuspidal } \\
\text { representations of } \\
\mathrm{GL}_{n}(E) \text { of depth zero }
\end{array}\right\} . \\
& (\tau, z) \longrightarrow \operatorname{Ind}_{\widetilde{K}_{0}}^{G} \widetilde{\tau}_{z} \\
& \left(\tau, \omega_{\pi}\left(\varpi_{E} 1_{n}\right)\right) \longleftarrow \pi
\end{aligned}
$$

From now on we denote the representation $\tau$ by $\rho_{0}$. So $\rho_{0}$ is an irreducible 
cuspidal representation of $\mathrm{GL}_{n}\left(k_{E}\right)$ inflated to $K_{0}=\mathrm{GL}_{n}\left(\mathfrak{O}_{E}\right)$.

\subsection{Siegel parahoric subgroup}

The Siegel parahoric subgroup $\mathfrak{P}$ of $\mathrm{U}(n, n)$ is defined by:

$$
\mathfrak{P}=\left[\begin{array}{cc}
\mathrm{GL}_{n}\left(\mathfrak{O}_{E}\right) & \mathrm{M}_{n}\left(\mathfrak{O}_{E}\right) \\
\mathrm{M}_{n}\left(\mathbf{p}_{E}\right) & \mathrm{GL}_{n}\left(\mathfrak{O}_{E}\right)
\end{array}\right] \cap \mathrm{U}(n, n) .
$$

Let

$$
\bar{U}=\left\{\left[\begin{array}{ll}
1 & 0 \\
X & 1
\end{array}\right] \mid X \in \mathrm{M}_{n}(E), X+{ }^{t} \bar{X}=0\right\} .
$$

We have $\mathfrak{P}=(\mathfrak{P} \cap \bar{U})(\mathfrak{P} \cap L)(\mathfrak{P} \cap U)$ (Iwahori factorization of $\mathfrak{P})$. Let us denote $(\mathfrak{P} \cap \bar{U})$ by $\mathfrak{P}_{-},(\mathfrak{P} \cap U)$ by $\mathfrak{P}_{+},(\mathfrak{P} \cap L)$ by $\mathfrak{P}_{0}$. Thus

$$
\begin{gathered}
\mathfrak{P}_{0}=\left\{\left[\begin{array}{cc}
a & 0 \\
0 & { }^{t} \bar{a}^{-1}
\end{array}\right] \mid a \in \mathrm{GL}_{n}\left(\mathfrak{O}_{E}\right)\right\}, \\
\mathfrak{P}_{+}=\left\{\left[\begin{array}{cc}
1 & X \\
0 & 1
\end{array}\right] \mid X \in \mathrm{M}_{n}\left(\mathfrak{O}_{E}\right), X+{ }^{t} \bar{X}=0\right\}, \\
\mathfrak{P}_{-}=\left\{\left[\begin{array}{ll}
1 & 0 \\
X & 1
\end{array}\right] \mid X \in \mathrm{M}_{n}\left(\mathfrak{O}_{E}\right), X+{ }^{t} \bar{X}=0\right\} .
\end{gathered}
$$

\subsection{Representation of Siegel parahoric subgroup}

Let us recall that the Siegel parahoric subgroup $\mathfrak{P}$ of $\mathrm{U}(n, n)$ is defined as:

$$
\mathfrak{P}=\left[\begin{array}{cc}
\mathrm{GL}_{n}\left(\mathfrak{O}_{E}\right) & \mathrm{M}_{n}\left(\mathfrak{O}_{E}\right) \\
\mathrm{M}_{n}\left(\mathbf{p}_{E}\right) & \mathrm{GL}_{n}\left(\mathfrak{O}_{E}\right)
\end{array}\right] \cap \mathrm{U}(n, n) .
$$


Recall by Iwahori factorization of $\mathfrak{P}$ we have $\mathfrak{P}=(\mathfrak{P} \cap \bar{U})(\mathfrak{P} \cap L)(\mathfrak{P} \cap U)=$ $\mathfrak{P}_{-} \mathfrak{P}_{0} \mathfrak{P}_{+}$.

As $\rho_{0}$ is a representation of $K_{0}$, it can also be viewed as a representation of $\mathfrak{P}_{0}$. This is because $\mathfrak{P}_{0} \cong K_{0}$. Let $V$ be the vector space associated with $\rho_{0}$. Now $\rho_{0}$ is extended to a map $\rho$ from $\mathfrak{P}$ to $G L(V)$ as follows. By Iwahori factorization, if $j \in \mathfrak{P}$ then $j$ can be written as $j_{-} j_{0} j_{+}$, where $j_{-} \in \mathfrak{P}_{-}, j_{+} \in \mathfrak{P}_{+}, j_{0} \in \mathfrak{P}_{0}$. Now the map $\rho$ on $\mathfrak{P}$ is defined as $\rho(j)=\rho_{0}\left(j_{0}\right)$.

Proposition 2.1. $\rho$ is a homomorphism from $\mathfrak{P}$ to $G L(V)$. So $\rho$ becomes a representation of $\mathfrak{P}$.

Proof. Let

$$
\mathfrak{P}_{0,1}=\left\{\left[\begin{array}{cc}
a & 0 \\
0 & { }^{t} \bar{a}^{-1}
\end{array}\right] \mid a \in K_{1}=1+\varpi \mathrm{M}_{n}\left(\mathfrak{O}_{E}\right)\right\} .
$$

Clearly, $\mathfrak{P}_{0,1} \cong K_{1}$. Now let us define $\mathfrak{P}_{1}=\mathfrak{P}_{-} \mathfrak{P}_{0,1} \mathfrak{P}_{+}$. We can observe clearly that $\mathfrak{P}$ is a subgroup of $\mathrm{U}(n, n) \cap \mathrm{GL}_{2 n}\left(\mathfrak{O}_{E}\right)$. We have the following group homomorphism:

$$
\phi: \mathfrak{P} \stackrel{\bmod \mathbf{p}_{E}}{\longrightarrow} P\left(k_{E}\right) .
$$

Here $P\left(k_{E}\right)$ is the Siegel parabolic subgroup of $\left\{g \in \mathrm{GL}_{2 n}\left(k_{E}\right) \mid{ }^{t} \bar{g} J g=J\right\}$. Now $P\left(k_{E}\right)=L\left(k_{E}\right) \ltimes U\left(k_{E}\right)$, where $L\left(k_{E}\right), U\left(k_{E}\right)$ are the Levi component and unipotent radical of the Siegel parabolic subgroup respectively.

$$
L\left(k_{E}\right)=\left\{\left[\begin{array}{cc}
a & 0 \\
0 & t \bar{a}^{-1}
\end{array}\right] \mid a \in \mathrm{GL}_{n}\left(k_{E}\right)\right\},
$$




$$
U\left(k_{E}\right)=\left\{\left[\begin{array}{cc}
1 & X \\
0 & 1
\end{array}\right] \mid X \in \mathrm{M}_{n}\left(k_{E}\right), X+{ }^{t} \bar{X}=0\right\}
$$

$\phi$ is a surjective homomorphism. Now let us find the inverse image of $U\left(k_{E}\right)$. Let $j \in \mathfrak{P}$ and $j=j_{-} j_{0} j_{+}$be the Iwahori factorization of $j$, where $j_{+} \in \mathfrak{P}_{+}, j_{-} \in$ $\mathfrak{P}_{-}, j_{0} \in \mathfrak{P}_{0}$. So $\phi(j) \in U\left(k_{E}\right) \Longleftrightarrow j_{0} \in \mathfrak{P}_{0,1}$. Therefore $\mathfrak{P}_{1}$ is the inverse image of $U\left(k_{E}\right)$ under $\phi$. So we have $\mathfrak{P} / \mathfrak{P}_{1} \cong P\left(k_{E}\right) / U\left(k_{E}\right) \cong L\left(k_{E}\right) \cong \mathrm{GL}_{n}\left(k_{E}\right)$. As $\rho(j)=\rho_{0}\left(j_{0}\right)$, so $\rho$ is a representation of $\mathfrak{P}$ which is lifted from representation $\rho_{0}$ of $\mathfrak{P}_{0}$ that is trivial on $\mathfrak{P}_{1}$. 


\section{Chapter 3}

\section{Structure of $\mathcal{H}\left(L, \rho_{0}\right)$ and $\mathcal{H}(G, \rho)$}

\subsection{Calculation of $N_{G}\left(\mathfrak{P}_{0}\right)$}

We set $G=\mathrm{U}(n, n)$. To describe $\mathcal{H}(G, \rho)$ we need to determine $N_{G}\left(\rho_{0}\right)$ which is given by

$$
N_{G}\left(\rho_{0}\right)=\left\{m \in N_{G}\left(\mathfrak{P}_{0}\right) \mid \rho_{0} \simeq \rho_{0}^{m}\right\}
$$

Further, to find out $N_{G}\left(\rho_{0}\right)$ we need to determine $N_{G}\left(\mathfrak{P}_{0}\right)$. To that end we shall calculate $N_{\mathrm{GL}_{n}(E)}\left(K_{0}\right)$. Let $Z=Z\left(\mathrm{GL}_{n}(E)\right)$. So $Z=\left\{\lambda 1_{n} \mid \lambda \in E^{\times}\right\}$.

Lemma 3.1. $N_{\mathrm{GL}_{n}(E)}\left(K_{0}\right)=K_{0} Z$.

Proof. By the Cartan decomposition, any $g \in \mathrm{GL}_{n}(E)$ can be written as

$$
g=k_{1}\left[\begin{array}{cccc}
\varpi_{E}^{l_{1}} & 0 & \ldots & 0 \\
0 & \varpi_{E}^{l_{2}} & \ldots & 0 \\
\vdots & \vdots & \ddots & 0 \\
0 & 0 & \ldots & \varpi_{E}^{l_{n}}
\end{array}\right] k_{2}
$$

where $k_{1}, k_{2} \in K_{0}$ and for certain $l_{1}, l_{2} \ldots l_{n} \in \mathbb{Z}$ with $l_{1} \leqslant l_{2} \leqslant \ldots l_{n}$. 
So we only need to determine the matrices $\left[\begin{array}{cccc}\varpi_{E}^{l_{1}} & 0 & \ldots & 0 \\ 0 & \varpi_{E}^{l_{2}} & \ldots & 0 \\ \vdots & \vdots & \ddots & 0 \\ 0 & 0 & \ldots & \varpi_{E}^{l_{n}}\end{array}\right]$ that normalize $K_{0}$. Let $A$ be one such matrix which normalizes $K_{0}$. So $A B A^{-1} \in K_{0}$ for all $B \in K_{0}$. Let the matrix $A$ be of form

$$
\left[\begin{array}{cccc}
\varpi_{E}^{l_{1}} & 0 & \ldots & 0 \\
0 & \varpi_{E}^{l_{2}} & \ldots & 0 \\
\vdots & \vdots & \ddots & 0 \\
0 & 0 & \ldots & \varpi_{E}^{l_{n}}
\end{array}\right]
$$

for certain $l_{1}, l_{2} \ldots l_{n} \in \mathbb{Z}$ with $l_{1} \leqslant l_{2} \leqslant \ldots l_{n}$. Now matrix $A^{-1}$ looks like

$$
\left[\begin{array}{cccc}
\varpi_{E}^{-l_{1}} & 0 & \ldots & 0 \\
0 & \varpi_{E}^{-l_{2}} & \ldots & 0 \\
\vdots & \vdots & \ddots & 0 \\
0 & 0 & \ldots & \varpi_{E}^{-l_{n}}
\end{array}\right]
$$

Let the matrix $B$ be of form $\left(b_{i j}\right)_{1 \leqslant i, j \leqslant n}$. So matrix $B$ looks like:

$$
\left[\begin{array}{cccc}
b_{11} & b_{12} & \ldots & b_{1 n} \\
b_{21} & b_{22} & \ldots & b_{2 n} \\
\vdots & \vdots & \ldots & \vdots \\
b_{n 1} & b_{n 2} & \ldots & b_{n n}
\end{array}\right]
$$

where $b_{i j} \in \mathfrak{O}_{E}$ for $1 \leqslant i, j \leqslant n$. Now $A B A^{-1} \in K_{0}$ for all $B \in K_{0}$. And that implies $\varpi_{E}^{l_{i}-l_{j}} b_{i j} \in \mathfrak{O}_{E}$ for $1 \leqslant i, j \leqslant n$. Choose a matrix $B$ in $K_{0}$ such that 
$b_{i i}=1$ for $1 \leqslant i \leqslant n, b_{i j}=0$ for $1 \leqslant i, j \leqslant n, i \neq 1, j \neq 2, i \neq j$ and $b_{12}=1$. So we have $\varpi_{E}^{l_{1}-l_{2}} \in \mathfrak{O}_{E}$. As only positive integral powers of $\varpi_{E}$ lie in $\mathfrak{O}_{E}$. Hence $l_{1} \geqslant l_{2}$. Similarly we can show that $l_{2} \geqslant l_{1}$. So $l_{1}=l_{2}$. We can show in a similar fashion that $l_{2}=l_{3}, l_{3}=l_{4}, \ldots, l_{n-1}=l_{n}$. Let us call $l_{1}=l_{2}=l_{3}=\cdots=l_{n}=r$ for some $r \in \mathbb{Z}$. Hence any matrix $\left[\begin{array}{cccc}\varpi_{E}^{l_{1}} & 0 & \ldots & 0 \\ 0 & \varpi_{E}^{l_{2}} & \ldots & 0 \\ \vdots & \vdots & \ddots & 0 \\ 0 & 0 & \ldots & \varpi_{E}^{l_{n}}\end{array}\right]$ in $N_{\mathrm{GL}_{n}(E)}\left(K_{0}\right)$ is of the form

$$
\left[\begin{array}{cccc}
\varpi_{E}^{r} & 0 & \cdots & 0 \\
0 & \varpi_{E}^{r} & \cdots & 0 \\
\vdots & \vdots & \ddots & 0 \\
0 & 0 & \ldots & \varpi_{E}^{r}
\end{array}\right]
$$

for some $r \in \mathbb{Z}$. So $N_{\mathrm{GL}_{n}(E)}\left(K_{0}\right)$ consists of all the matrices in $g \in \mathrm{GL}_{n}(E)$ such that

$$
g=M^{\prime}\left[\begin{array}{cccc}
\varpi_{E}^{r} & 0 & \ldots & 0 \\
0 & \varpi_{E}^{r} & \ldots & 0 \\
\vdots & \vdots & \ddots & 0 \\
0 & 0 & \ldots & \varpi_{E}^{r}
\end{array}\right] M^{\prime \prime}
$$

where $M^{\prime}, M^{\prime \prime} \in K_{0}, r \in \mathbb{Z}$. But we can see that

$$
\left[\begin{array}{cccc}
\varpi_{E}^{r} & 0 & \ldots & 0 \\
0 & \varpi_{E}^{r} & \ldots & 0 \\
\vdots & \vdots & \ddots & 0 \\
0 & 0 & \ldots & \varpi_{E}^{r}
\end{array}\right] \in Z\left(\mathrm{GL}_{n}(E)\right)
$$


Let $M=M^{\prime} M^{\prime \prime} u^{-1}$ for some $u \in \mathfrak{O}_{E}^{\times}$and let $u \varpi_{E}^{r}=a$ for some $a \in E^{\times}$. So now any matrix in $N_{\mathrm{GL}_{n}(E)}\left(K_{0}\right)$ is of form $g \in \mathrm{GL}_{n}(E)$ such that

$$
g=M\left[\begin{array}{cccc}
a & 0 & \ldots & 0 \\
0 & a & \ldots & 0 \\
\vdots & \vdots & \ddots & 0 \\
0 & 0 & \ldots & a
\end{array}\right]
$$

where $a \in E^{\times}, M \in K_{0}$. So we have $N_{\mathrm{GL}_{n}(E)}\left(K_{0}\right)=Z K_{0}=K_{0} Z$.

From now on let us denote $K_{0}$ by $K$. Now let us calculate $N_{G}\left(\mathfrak{P}_{0}\right)$. Note that $J=\left[\begin{array}{ll}0 & 1 \\ 1 & 0\end{array}\right] \in G$. Indeed, $J \in N_{G}\left(\mathfrak{P}_{0}\right)$. The center $Z\left(\mathfrak{P}_{0}\right)$ of $\mathfrak{P}_{0}$ is given by $Z\left(\mathfrak{P}_{0}\right)=\left\{\left[\begin{array}{cc}u 1 & 0 \\ 0 & \bar{u}^{-1} 1\end{array}\right] \mid u \in \mathfrak{O}_{E}^{\times}\right\}$.

The center $Z(L)$ of $L$ is given by

$$
Z(L)=\left\{\left[\begin{array}{cc}
a 1 & 0 \\
0 & \bar{a}^{-1} 1
\end{array}\right] \mid a \in E^{\times}\right\} .
$$

Proposition 3.2. $N_{G}\left(\mathfrak{P}_{0}\right)=\left\langle\mathfrak{P}_{0} Z(L), J\right\rangle=\mathfrak{P}_{0} Z(L) \rtimes\langle J\rangle$.

Proof. It easy to see that $N_{G}\left(\mathfrak{P}_{0}\right) \leqslant N_{G}\left(Z\left(\mathfrak{P}_{0}\right)\right)$. Now suppose $g=\left[\begin{array}{ll}A & B \\ C & D\end{array}\right] \in$ $N_{G}\left(Z\left(\mathfrak{P}_{0}\right)\right)$, where $A, B, C, D \in \mathrm{M}_{n}(E)$. Let us choose $u \in \mathfrak{O}_{E}^{\times}$such that $u \neq \bar{u}^{-1}$. Now such a $u$ exists in $\mathfrak{O}_{E}^{\times}$. Because if $u=\bar{u}^{-1}$ for all $u \in \mathfrak{O}_{E}^{\times}$then $\bar{u}=u^{-1}$ for all $u \in \mathfrak{O}_{E}^{\times}$. But $\mathfrak{O}_{E}^{\times} \cap F^{\times}=\mathfrak{O}_{F}^{\times}$. Therefore $u=u^{-1}$ for all $u \in \mathfrak{O}_{F}^{\times}$or $u^{2}=1$ for all $u \in \mathfrak{O}_{F}^{\times}$which is a contradiction. 


$$
\begin{gathered}
\text { As }\left[\begin{array}{cc}
A & B \\
C & D
\end{array}\right] \in N_{G}\left(Z\left(\mathfrak{P}_{0}\right)\right) \\
{\left[\begin{array}{cc}
A & B \\
C & D
\end{array}\right]\left[\begin{array}{cc}
u 1 & 0 \\
0 & \bar{u}^{-1} 1
\end{array}\right]\left[\begin{array}{cc}
A & B \\
C & D
\end{array}\right]^{-1}=\left[\begin{array}{cc}
v 1 & 0 \\
0 & \bar{v}^{-1} 1
\end{array}\right]}
\end{gathered}
$$

for some $v \in \mathfrak{O}_{E}^{\times}$. The left and right hand sides must have the same eigenvalues. So $u=v$ or $\bar{v}^{-1}$. Let $u=v$. Then we have

$$
\begin{gathered}
{\left[\begin{array}{ll}
A & B \\
C & D
\end{array}\right]\left[\begin{array}{cc}
u 1 & 0 \\
0 & \bar{u}^{-1} 1
\end{array}\right]\left[\begin{array}{cc}
A & B \\
C & D
\end{array}\right]^{-1}=\left[\begin{array}{cc}
v 1 & 0 \\
0 & \bar{v}^{-1} 1
\end{array}\right]} \\
\Longrightarrow\left[\begin{array}{cc}
A u & B \bar{u}^{-1} \\
C u & D \bar{u}^{-1}
\end{array}\right]=\left[\begin{array}{cc}
A v & B v \\
C \bar{v}^{-1} & D \bar{u}^{-1}
\end{array}\right] .
\end{gathered}
$$

As $u=v$, so $A u=A v, D \bar{u}^{-1}=D \bar{v}^{-1}$. Now as $u \neq \bar{v}^{-1}$ (i.e $v \neq \bar{u}^{-1}$ ), from the above matrix relation we can see that $B \bar{u}^{-1}=B v, C u=C \bar{v}^{-1}$ for arbitrary matrices $B$ and $C$. So this would imply that $B=C=0$. In a similar way, we can show that if $u=\bar{v}^{-1}$ then $A=D=0$. Hence any element of $N_{G}\left(Z\left(\mathfrak{P}_{0}\right)\right)$ is of the form $\left[\begin{array}{ll}A & 0 \\ 0 & D\end{array}\right]$ or $\left[\begin{array}{ll}0 & B \\ C & 0\end{array}\right]$ with $A, B, C, D \in \mathrm{GL}_{n}(E)$. As $N_{G}\left(\mathfrak{P}_{0}\right) \leqslant N_{G}\left(Z\left(\mathfrak{P}_{0}\right)\right)$, so any element which normalizes $\mathfrak{P}_{0}$ is also of the form $\left[\begin{array}{cc}A & 0 \\ 0 & D\end{array}\right]$ or $\left[\begin{array}{cc}0 & B \\ C & 0\end{array}\right]$ with $A, B, C, D \in \mathrm{GL}_{n}(E)$.

$$
\begin{gathered}
\text { If }\left[\begin{array}{cc}
A & 0 \\
0 & D
\end{array}\right] \text { normalizes } \mathfrak{P}_{0} \text { then } \\
{\left[\begin{array}{cc}
A & 0 \\
0 & D
\end{array}\right]\left[\begin{array}{cc}
a & 0 \\
0 & { }^{t} \bar{a}^{-1}
\end{array}\right]\left[\begin{array}{cc}
A^{-1} & 0 \\
0 & D^{-1}
\end{array}\right] \in \mathfrak{P}_{0} \text { for all } a \in K .}
\end{gathered}
$$




$$
\Longrightarrow\left[\begin{array}{cc}
A a A^{-1} & 0 \\
0 & D^{t} \bar{a}^{-1} D^{-1}
\end{array}\right] \in \mathfrak{P}_{0} \text { for all } a \in K .
$$

Hence $A a A^{-1}, D^{t} \bar{a}^{-1} D^{-1} \in K$ for all $a \in K$. So this implies that $A, D \in$ $N_{\mathrm{GL}_{n}(E)}(K)=Z K=K Z$ from lemma 3.1 and also ${ }^{t} \overline{\left(A a A^{-1}\right)^{-1}}=D^{t} \bar{a}^{-1} D^{-1}$ for all $a \in K$. If ${ }^{t} \overline{\left(A a A^{-1}\right)}-1=D^{t} \bar{a}^{-1} D^{-1}$ for all $a \in K$ then ${ }^{t} \bar{A}^{-1 t} \bar{a}^{-1 t} \bar{A}=$ $D^{t} \bar{a}^{-1} D^{-1}$ for all $a \in K \Longrightarrow A={ }^{t} \bar{D}^{-1}$ (i.e $D={ }^{t} \bar{A}^{-1}$ ). And as $A \in Z K$, so $A=z k$ for some $z \in Z, k \in K$. Hence

$$
\left[\begin{array}{cc}
A & 0 \\
0 & D
\end{array}\right]=\left[\begin{array}{cc}
z k & 0 \\
0 & t \overline{(z k)^{-1}}
\end{array}\right]
$$

Similarly, we can show that if $\left[\begin{array}{ll}0 & B \\ C & 0\end{array}\right]$ normalizes $\mathfrak{P}_{0}$ then

$$
\left[\begin{array}{ll}
0 & B \\
C & 0
\end{array}\right]=\left[\begin{array}{cc}
0 & z^{\prime} k^{\prime} \\
t \overline{\left(z^{\prime} k^{\prime}\right)^{-1}} & 0
\end{array}\right] \text { for some } z^{\prime} \in Z, k^{\prime} \in K \text {. }
$$

If $\left[\begin{array}{ll}A & 0 \\ 0 & D\end{array}\right] \in N_{G}\left(\mathfrak{P}_{0}\right)$, we have shown that it looks like $\left[\begin{array}{cc}z k & 0 \\ 0 & { }^{t}(z k)^{-1}\end{array}\right]$ and if $\left[\begin{array}{cc}0 & B \\ C & 0\end{array}\right] \in N_{G}\left(\mathfrak{P}_{0}\right)$, we have shown that it looks like $\left[\begin{array}{cc}0 & z^{\prime} k^{\prime} \\ t \overline{\left(z^{\prime} k^{\prime}\right)^{-1}} & 0\end{array}\right]$ where $z, z^{\prime} \in Z, k, k^{\prime} \in K$. We know that $J \in N_{G}\left(\mathfrak{P}_{0}\right)$ and as $\left[\begin{array}{ll}0 & B \\ C & 0\end{array}\right] J=\left[\begin{array}{ll}B & 0 \\ 0 & C\end{array}\right]$, so $N_{G}\left(\mathfrak{P}_{0}\right)=\left\langle J ;\left[\begin{array}{cc}z k & 0 \\ 0 & { }^{t} \overline{(z k)^{-1}}\end{array}\right] \mid z \in Z, k \in K\right\rangle=\mathfrak{P}_{0} Z(L) \rtimes\langle J\rangle$. 


\section{2 $N_{G}\left(\rho_{0}\right):$ unramified case}

We now calculate $N_{G}\left(\rho_{0}\right)$ in the unramified case. This will help in determining the structure of $\mathcal{H}(G, \rho)$.

As $\rho_{0}$ is an irreducible cuspidal representation of $\mathrm{GL}_{n}\left(k_{E}\right)$, there exists a regular character $\theta$ of $l^{\times}$(where $l$ is a degree $n$ extension of $k_{E}$ ) such that $\rho_{0}=\tau_{\theta}$. We have $k_{E}=\mathbb{F}_{q^{2}}$. So $l=\mathbb{F}_{q^{2 n}}$.

Let $\Gamma=\operatorname{Gal}\left(l / k_{E}\right)$. The group $\Gamma$ is generated by the Frobenius map $\Phi$ given by $\Phi(\lambda)=\lambda^{q^{2}}$ for $\lambda \in l$. Here $\Phi^{n}(\lambda)=\lambda^{q^{2 n}}=\lambda$ (since $l^{\times}$is a cyclic group of order $\left.q^{2 n}-1\right) \Longrightarrow \Phi^{n}=1$.

Let us look at the action of $\Gamma$ on $\operatorname{Hom}\left(l^{\times}, \mathbb{C}^{\times}\right)$. For $\gamma \in \Gamma$ and $\theta \in \operatorname{Hom}\left(l^{\times}, \mathbb{C}^{\times}\right)$, $\gamma$ acts on $\theta$ by $\gamma \cdot \theta(\lambda)=\theta(\gamma(\lambda))$. Here $\gamma \cdot \theta$ is also represented by $\theta^{\gamma}$.

We say a character $\theta$ is regular character if $\operatorname{stab}_{\Gamma}(\theta)=\left\{\gamma \in \Gamma \mid \theta^{\gamma}=\theta\right\}=1$. So if $\theta$ is regular character of $l^{\times}$then $\theta^{\gamma}=1 \Longrightarrow \gamma=1$. And also for two regular characters $\theta$ and $\theta^{\prime}$ we have $\tau_{\theta} \simeq \tau_{\theta^{\prime}} \Longleftrightarrow$ there exists $\gamma \in \Gamma$ such that $\theta^{\gamma}=\theta^{\prime}$.

As we are in the unramified case, $\operatorname{so} \operatorname{Gal}\left(k_{E} / k_{F}\right) \cong \operatorname{Gal}(E / F)$. Let $\iota: \mathrm{GL}_{n}\left(k_{E}\right)$ $\longrightarrow \mathrm{GL}_{n}\left(k_{E}\right)$ be a group homomorphism given by: $\iota(g)={ }^{t} \bar{g}^{-1}$. Let us denote $\tau_{\theta} \circ \iota$ by $\tau_{\theta}^{\iota}$. So $\tau_{\theta}^{\iota}(g)=\tau_{\theta}(\iota(g))=\tau_{\theta}\left({ }^{t} \bar{g}^{-1}\right)$ for $g \in \mathrm{GL}_{n}\left(k_{E}\right)$. We also denote $\overline{\tau_{\theta}}(g)$ for $\tau_{\theta}(\bar{g})$ for $g \in \mathrm{GL}_{n}\left(k_{E}\right)$. It can be observed clearly as $\theta$ is a character of $l^{\times}$, so $\theta\left(\lambda^{m}\right)=\theta^{m}(\lambda)$ for $m \in \mathbb{Z}, \lambda \in l^{\times}$.

Let $\tau_{\theta}^{\vee}$ be the dual representation of $\tau_{\theta}$. Let $V$ be the vector space corresponding to $\tau_{\theta}$ which is finite dimensional. Choose a basis $\left\{v_{1}, v_{2}, \ldots v_{n}\right\}$ of the vector space $V$. The dual basis $\left\{v_{1}^{*}, v_{2}^{*}, \ldots v_{n}^{*}\right\}$ for the dual space $V^{*}$ of $V$ can be constructed such that $v_{i}^{*}\left(v_{j}\right)=\delta_{i j}$ for $1 \leqslant i, j \leqslant n$. Suppose with respect to the above basis $\left\{v_{1}, v_{2}, \ldots v_{n}\right\}, \tau_{\theta}\left(g^{-1}\right)$ represents matrix $A$ and with respect to the dual basis $\left\{v_{1}^{*}, v_{2}^{*}, \ldots v_{n}^{*}\right\}, \tau_{\theta}^{\vee}(g)$ represents matrix $B$, then $A={ }^{t} B$. 
From Propn. 3.5 in [7] we have $\bar{\tau}_{\theta} \simeq \tau_{\theta^{q}}$ and from Propn. 3.4 in [7] we have $\tau_{\theta}^{\vee} \simeq \tau_{\theta^{-1}}$

Proposition 3.3. Let $\theta$ be a regular character of $l^{\times}$. Then $\tau_{\theta}^{\iota} \simeq \tau_{\theta} \Longleftrightarrow \theta^{\gamma}=\theta^{-q}$ for some $\gamma \in \operatorname{Gal}\left(l / k_{E}\right)$.

Proof. $\Longrightarrow$ As $\tau_{\theta}^{\iota} \simeq \tau_{\theta}$, so $\chi_{\tau_{\theta}^{\iota}}(g)=\chi_{\tau_{\theta}}(g)$ for $g \in \mathrm{GL}_{n}\left(k_{E}\right)$. But $\chi_{\tau_{\theta}^{\iota}}(g)=$ $\chi_{\tau_{\theta}}\left({ }^{t} \bar{g}^{-1}\right)$, since $\chi_{\tau_{\theta}^{\iota}}(g)=\chi_{\tau_{\theta}}(\iota(g))$ for $g \in \mathrm{GL}_{n}\left(k_{E}\right)$. As we know from the above discussion that $\tau_{\theta}^{\vee}(g)=\left(\tau_{\theta}\left(g^{-1}\right)\right)^{t}$, so $\operatorname{trace}\left(\tau_{\theta}^{\vee}(g)\right)=\operatorname{trace}\left(\tau_{\theta}\left(g^{-1}\right)\right)^{t}$. Now $\operatorname{trace}\left(\tau_{\theta}\left(g^{-1}\right)\right)=\operatorname{trace}\left(\tau_{\theta}\left(g^{-1}\right)\right)^{t}$ as the trace of the matrix and it's transpose are same. So we have $\operatorname{trace}\left(\tau_{\theta}\left(g^{-1}\right)\right)=\operatorname{trace}\left(\tau_{\theta}^{\vee}(g)\right)$. Let us choose $h \in \operatorname{GL}_{n}\left(k_{E}\right)$ such that $h^{-1 t} g^{-1} h=g^{-1}$. So, $\chi_{\tau_{\theta}^{\vee}}(g)=\chi_{\tau_{\theta}}\left(g^{-1}\right)=\chi_{\tau_{\theta}}\left(h^{-1 t} g^{-1} h\right)=\chi_{\tau_{\theta}}\left({ }^{t} g^{-1}\right)$. Let us denote $\tau_{\theta}^{\eta}(g)$ for $\tau_{\theta}(\eta(g))$ where $\eta: g \longrightarrow{ }^{t} g^{-1}$ is a group automorphism of $\mathrm{GL}_{n}\left(k_{E}\right)$. Hence $\chi_{\tau_{\theta}^{\eta}}(g)=\chi_{\tau_{\theta}}\left({ }^{t} g^{-1}\right)$. But we have already shown before that $\chi_{\tau_{\theta}}\left({ }^{t} g^{-1}\right)=\chi_{\tau_{\theta}^{\vee}}(g)$. so $\chi_{\tau_{\theta}^{\vee}}(g)=\chi_{\tau_{\theta}^{\eta}}(g)$. This implies $\tau_{\theta}^{\vee} \simeq \tau_{\theta}^{\eta}$. Hence $\tau_{\theta}^{\iota}=\bar{\tau}_{\theta}^{\eta} \simeq \bar{\tau}_{\theta}^{\vee} \simeq \tau_{\theta^{q}}^{\vee} \simeq \tau_{\theta^{-q}}\left(\right.$ since $\left.\tau_{\theta}^{\vee} \simeq \tau_{\theta}^{\eta}, \bar{\tau}_{\theta} \simeq \tau_{\theta^{q}}, \tau_{\theta}^{\vee} \simeq \tau_{\theta^{-1}}\right)$. Now from the hypothesis of Propn. we know that $\tau_{\theta}^{\iota} \simeq \tau_{\theta}$, so this implies $\tau_{\theta} \simeq \tau_{\theta^{-q}}$ (since $\left.\tau_{\theta}^{\iota} \simeq \tau_{\theta^{-q}}\right)$. But as $\theta$ is a regular character $\theta^{\gamma}=\theta^{-q}$ for some $\gamma \in \Gamma=\operatorname{Gal}\left(l / k_{E}\right)$ where $\left[l: k_{E}\right]=n$.

$\Longleftarrow$ Now we can reverse the arguments and show that if $\theta^{\gamma}=\theta^{-q}$ for some $\gamma \in \Gamma=\operatorname{Gal}\left(l / k_{E}\right)$ then $\tau_{\theta}^{\iota} \simeq \tau_{\theta^{-q}}$.

Proposition 3.4. If $\theta$ is a regular character of $l^{\times}$such that $\theta^{\gamma}=\theta^{-q}$ for some $\gamma \in \Gamma$ then $n$ is odd. Conversely, if $n=2 m+1$ is odd and $\theta$ is a regular character of $l^{\times}$then $\theta^{\Phi^{m+1}}=\theta^{-q}$.

Proof. $\Longrightarrow$ Suppose $\theta$ is a regular character of $l^{\times}$such that $\theta^{\gamma}=\theta^{-q}$ for some $\gamma \in \Gamma$. We know that $\Gamma=<\Phi>$ where $\Phi: l \longrightarrow l$ is the Frobenius map given by 
$\Phi(\lambda)=\lambda^{q^{2}}$ for $\lambda \in l$. Now $\Phi^{n}(\lambda)=\lambda^{q^{2 n}}=\lambda$ for $\lambda \in l \Longrightarrow \Phi^{n}=1$. Now we have $\left(\theta^{-q}\right)^{\gamma}=\left(\theta^{\gamma}\right)^{-q}$. Hence $(\theta)^{\gamma^{2}}=\left(\theta^{\gamma}\right)^{\gamma}=\left(\theta^{-q}\right)^{\gamma}=\left(\theta^{\gamma}\right)^{-q}=\left(\theta^{-q}\right)^{-q}=\theta^{q^{2}}=\theta^{\Phi}$. Now $\theta^{q^{2}}=\theta^{\Phi}$ because for $\lambda \in l^{\times}, \theta^{q^{2}}(\lambda)=\theta\left(\lambda^{q^{2}}\right)=\theta(\Phi(\lambda))=\theta^{\Phi}(\lambda)$. As $\theta$ is a regular character and $(\theta)^{\gamma^{2}}=\theta^{\Phi}$, so $\gamma^{2}=\Phi$. Let $\Phi$ be a generator of $\Gamma$ and $\gamma^{2}=\Phi$. So $\gamma$ is also a generator of $\Gamma$.

Hence order of $\gamma^{2}=$ order of $\Phi \Longrightarrow \frac{n}{g \cdot c . d(2, n)}=n \Longrightarrow \operatorname{g.c.d}(2, n)=1$. So $n$ is odd.

$\Longleftarrow$ Suppose $n$ is odd. Let $n=2 m+1$ where $m \in \mathbb{N}$. Now

$$
\operatorname{Hom}\left(l^{\times}, \mathbb{C}^{\times}\right) \cong l^{\times}
$$

So $\operatorname{Hom}\left(l^{\times}, \mathbb{C}^{\times}\right)$is a cyclic group of order $\left(q^{2 n}-1\right)$. Hence for every divisor $d$ of $\left(q^{2 n}-1\right)$, there exists an element in $\operatorname{Hom}\left(l^{\times}, \mathbb{C}^{\times}\right)$of order $d$. As $\left(q^{n}+1\right)$ is a divisor of $\left(q^{2 n}-1\right)$, hence there exists an element $\theta$ in $\operatorname{Hom}\left(l^{\times}, \mathbb{C}^{\times}\right)$of order $\left(q^{n}+1\right)$. Hence $\theta^{q^{n}+1}=1 \Longrightarrow \theta^{q^{n}}=\theta^{-1} \Longrightarrow \theta^{q^{n+1}}=\theta^{-q} \Longrightarrow \theta^{q^{2 m+2}}=\theta^{-q} \Longrightarrow$ $\theta^{\left(q^{2}\right)^{m+1}}=\theta^{-q} \Longrightarrow \theta^{\Phi^{m+1}}=\theta^{-q} \Longrightarrow \theta^{\gamma}=\theta^{-q}$, where $\gamma=\Phi^{m+1} \in \Gamma$.

Now we claim that $\theta$ is a regular character in $\operatorname{Hom}\left(l^{\times}, \mathbb{C}^{\times}\right)$. suppose $\theta^{\gamma}=\theta$ for some $\gamma \in \Gamma$. Let $\gamma=\Phi^{k}$ for some $k \in \mathbb{N}$. So we have $\theta^{\Phi^{k}}=\theta$. But $\theta^{\Phi}=\theta^{q^{2}}$, hence $\theta^{q^{2 k}}=\theta$. That implies $\theta^{q^{2 k}-1}=1$. As $\theta$ has order $\left(q^{n}+1\right)$, so $\left(q^{n}+1\right) \mid\left(q^{2 k}-1\right)$. Let $l=2 k$, so we have $\left(q^{n}+1\right) \mid\left(q^{l}-1\right)$. If $l<n$ then it is a contradiction to the fact that $\left(q^{n}+1\right) \mid\left(q^{l}-1\right)$. Hence $l>n$. Now by applying Euclidean Algorithm for the integers $l, n$ we have $l=n d+r$ for some $0 \leqslant r<n$ and $d>0$ where $r, d \in \mathbb{Z}$. Now $d \neq 0$, because if $d=0$ then $l=r$ and that means $l<n$ which is a contradiction. So $d \in \mathbb{N}$. As we have $\left(q^{n}+1\right)\left|\left(q^{l}-1\right) \Longrightarrow\left(q^{n}+1\right)\right|$ $\left(\left(q^{l}-1\right)+\left(q^{n}+1\right)\right) \Longrightarrow\left(q^{n}+1\right)\left|\left(q^{l}+q^{n}\right) \Longrightarrow\left(q^{n}+1\right)\right| q^{n}\left(q^{r} \cdot q^{n(d-1)}+1\right)$. Now as $q^{n}$ and $\left(q^{n}+1\right)$ are relatively prime, so $\left(q^{n}+1\right) \mid\left(q^{r} \cdot q^{n(d-1)}+1\right) \Longrightarrow$ $\left(q^{n}+1\right)\left|\left(\left(q^{r} \cdot q^{n(d-1)}+1\right)-\left(q^{n}+1\right)\right) \Longrightarrow\left(q^{n}+1\right)\right| q^{n}\left(q^{r} \cdot q^{n(d-2)}-1\right)$. As $q^{n}$ 
and $\left(q^{n}+1\right)$ are relatively prime, so $\left(q^{n}+1\right) \mid\left(q^{r} \cdot q^{n(d-2)}-1\right)$. So continuing the above process we get, $\left(q^{n}+1\right) \mid\left(q^{r}+1\right)$ if $d$ is odd or $\left(q^{n}+1\right) \mid\left(q^{r}-1\right)$ if $d$ is even. But degree of $\left(q^{n}+1\right)$ is greater than degree of $\left(q^{r}+1\right)$ as $r<n$. So $r$ has to be equal to 0 and $l=2 k=n d+r=n d$. And that implies $2 \mid n d$. But $n$ is odd so $2 \mid d$. Now this means that $d$ is even and hence $\left(q^{n}+1\right) \mid\left(q^{r}-1\right)$. And $\left(q^{n}+1\right) \mid\left(q^{r}-1\right)$ is not possible because $r=0$. So we have $2 k=n d \Longrightarrow n \mid 2 k$. But as $n$ is odd this implies $n \mid k$. And this further implies $k=n p$ for some $p \in \mathbb{N}$. So $\gamma=\Phi^{k}=\Phi^{n p}=1 \Longrightarrow \theta$ is regular character.

Combining Propn. 3.3 and Propn. 3.4, we have the following Propn.

Proposition 3.5. Let $\theta$ is a regular character of $l^{\times}$. Then $\tau_{\theta}^{\iota} \simeq \tau_{\theta} \Longleftrightarrow n$ is odd.

We know that $\rho_{0}$ is an irreducible cuspidal representation of $K$. But $K \cong \mathfrak{P}_{0}$. So $\rho_{0}$ can be viewed as a representation of $\mathfrak{P}_{0}$. Now let us compute $N_{G}\left(\rho_{0}\right)$, where $N_{G}\left(\rho_{0}\right)=\left\{m \in N_{G}\left(\mathfrak{P}_{0}\right) \mid \rho_{0} \simeq \rho_{0}^{m}\right\}$. Let $m \in N_{G}\left(\mathfrak{P}_{0}\right)$. Hence $m$ is either $J$ or $m$ is of the form $\left[\begin{array}{cc}z k & 0 \\ 0 & { }^{t}(\overline{z k)}-1\end{array}\right]$ for some $z \in Z, k \in K$.

Proposition 3.6. If $m=\left[\begin{array}{cc}z k & 0 \\ 0 & { }^{t} \overline{(z k)}-1\end{array}\right]$ for some $z \in Z, k \in K$ then $\rho_{0}{ }^{m} \simeq \rho_{0}$. Proof. As $\rho_{0}$ is an irreducible cuspidal representation of $K$, so $K$ normalizes $\rho_{0}$. Clearly, $Z$ normalizes $\rho_{0}$. Thus $Z K$ normalizes $\rho_{0}$. As $\rho_{0}$ can also be viewed as a representation of $\mathfrak{P}_{0}$, so $\rho_{0}{ }^{m} \simeq \rho_{0}$ where $m=\left[\begin{array}{cc}z k & 0 \\ 0 & { }^{t} \overline{(z k)}-1\end{array}\right]$ for some $z \in Z, k \in K$

Proposition 3.7. If $m=J=\left[\begin{array}{ll}0 & 1 \\ 1 & 0\end{array}\right]$ then $\rho_{0}{ }^{m} \simeq \rho_{0}$ only when $n$ is odd. 
Proof. We know that $\iota: a \longrightarrow{ }^{t} \bar{a}^{-1}$ is a group homomorphism of $\mathrm{GL}_{n}\left(k_{E}\right)$. Now $\iota: a \longrightarrow{ }^{t} \bar{a}^{-1}$ can be inflated to a group homomorphism of $\mathrm{GL}_{n}\left(\mathfrak{O}_{E}\right)$. Further, $\iota$ can be viewed as a group homomorphism from $\mathfrak{P}_{0}$ to $\mathfrak{P}_{0}$ given by:

$$
\iota\left(\left[\begin{array}{cc}
a & 0 \\
0 & { }^{t} \bar{a}^{-1}
\end{array}\right]\right)=\left[\begin{array}{cc}
{ }^{t} \bar{a}^{-1} & 0 \\
0 & a
\end{array}\right]
$$

where $a \in \mathrm{GL}_{n}\left(\mathfrak{O}_{E}\right)$. Let $g=\left[\begin{array}{cc}a & 0 \\ 0 & { }^{t} \bar{a}^{-1}\end{array}\right]$. If $m=J$ then $\rho_{0}{ }^{m}(g)=\rho_{0}\left(J g J^{-1}\right)=$ $\rho_{0}\left(\left[\begin{array}{cc}t \bar{a}^{-1} & 0 \\ 0 & a\end{array}\right]\right)=\rho_{0}(\iota(g))=\rho_{0}^{\iota}(g)$. So $\rho_{0}{ }^{m}(g)=\rho_{0}^{\iota}(g)$ for $g \in \mathfrak{P}_{0} \Longrightarrow \rho_{0}{ }^{m}=$ $\rho_{0}^{\iota}$. But from the hypothesis of Propn., we know that $\rho_{0}^{m} \simeq \rho_{0}$. So we have $\rho_{0} \simeq \rho_{0}^{\iota}$. Now from Propn. 3.5, $\rho_{0} \simeq \rho_{0}^{\iota}=\rho_{0}^{m} \Longleftrightarrow n$ is odd.

Thus we have the following conclusion about $N_{G}\left(\rho_{0}\right)$ for the unramified case: If $n$ is even then $N_{G}\left(\rho_{0}\right)=Z(L) \mathfrak{P}_{0}$ and if $n$ is odd then $N_{G}\left(\rho_{0}\right)=Z(L) \mathfrak{P}_{0} \rtimes$ $\langle J\rangle$.

\section{3 $N_{G}\left(\rho_{0}\right):$ ramified case}

Now that we have calculated $N_{G}\left(\mathfrak{P}_{0}\right)$, let us calculate $N_{G}\left(\rho_{0}\right)$ for the ramified case which would help us in determining the structure of $\mathcal{H}(G, \rho)$ in the ramified case.

As in section 3.2, $\rho_{0}=\tau_{\theta}$ for some regular character $\theta$ of $l^{\times}$(where $l$ is a degree $n$ extension of $k_{E}$ ). We have $k_{E}=\mathbb{F}_{q}$. So $l=\mathbb{F}_{q^{n}}$.

Let $\Gamma=\operatorname{Gal}\left(l / k_{E}\right)$. The group $\Gamma$ is generated by Frobenius map $\Phi$ given by $\Phi(\lambda)=\lambda^{q}$ for $\lambda \in l$. Here $\Phi^{n}(\lambda)=\lambda^{q^{n}}=\lambda$ (since $l^{\times}$is a cyclic group of order 
$\left.q^{n}-1\right) \Longrightarrow \Phi^{n}=1$.

For $\gamma \in \Gamma$ and $\theta \in \operatorname{Hom}\left(l^{\times}, \mathbb{C}^{\times}\right), \gamma$ acts on $\theta$ by $\gamma \cdot \theta(\lambda)=\theta(\gamma(\lambda))$. Here $\gamma \cdot \theta$ is also represented by $\theta^{\gamma}$.

As we are in the ramified case, so $\operatorname{Gal}\left(k_{E} / k_{F}\right)=1$. So $\bar{g}=g$ for $g \in k_{E}$. Let $\iota: \mathrm{GL}_{n}\left(k_{E}\right) \longrightarrow \mathrm{GL}_{n}\left(k_{E}\right)$ be a group homomorphism given by: $\iota(g)={ }^{t} \bar{g}^{-1}=$ ${ }^{t} g^{-1}$. Let us denote $\tau_{\theta} \circ \iota$ by $\tau_{\theta}{ }^{\iota}$. So $\tau_{\theta}{ }^{\iota}(g)=\tau_{\theta}(\iota(g))=\tau_{\theta}\left({ }^{t} \bar{g}^{-1}\right)=\tau_{\theta}\left({ }^{t} g^{-1}\right)$ for $g \in \mathrm{GL}_{n}\left(k_{E}\right)$. We also denote $\overline{\tau_{\theta}}(g)$ for $\tau_{\theta}(\bar{g})$ for $g \in \mathrm{GL}_{n}\left(k_{E}\right)$. But $\overline{\tau_{\theta}}(g)=\tau_{\theta}(\bar{g})=$ $\tau_{\theta}(g)$. It can be observed clearly as $\theta$ is a character of $l^{\times}$, so $\theta\left(\lambda^{m}\right)=\theta^{m}(\lambda)$ for $m \in \mathbb{Z}, \lambda \in l^{\times}$.

Proposition 3.8. Let $\theta$ be a regular character of $l^{\times}$. Then $\tau_{\theta}^{\iota} \simeq \tau_{\theta} \Longleftrightarrow \theta^{\gamma}=\theta^{-1}$ for some $\gamma \in \operatorname{Gal}\left(l / k_{E}\right)$.

Proof. $\Longrightarrow$ As $\tau_{\theta}^{\iota} \simeq \tau_{\theta}$, so $\chi_{\tau_{\theta}^{\iota}}(g)=\chi_{\tau_{\theta}}(g)$ for $g \in \mathrm{GL}_{n}\left(k_{E}\right)$. But $\chi_{\tau_{\theta}^{\iota}}(g)=$ $\chi_{\tau_{\theta}}\left({ }^{t} g^{-1}\right)$, since $\chi_{\tau_{\theta}^{\iota}}(g)=\chi_{\tau_{\theta}}(\iota(g))$ for $g \in \mathrm{GL}_{n}\left(k_{E}\right)$. As we know from the above discussion that $\tau_{\theta}^{\vee}(g)=\left(\tau_{\theta}\left(g^{-1}\right)\right)^{t}$, so $\operatorname{trace}\left(\tau_{\theta}^{\vee}(g)\right)=\operatorname{trace}\left(\tau_{\theta}\left(g^{-1}\right)\right)^{t}$. Now $\operatorname{trace}\left(\tau_{\theta}\left(g^{-1}\right)\right)=\operatorname{trace}\left(\tau_{\theta}\left(g^{-1}\right)\right)^{t}$ as the trace of the matrix and it's transpose are same. So we have $\operatorname{trace}\left(\tau_{\theta}\left(g^{-1}\right)\right)=\operatorname{trace}\left(\tau_{\theta}^{\vee}(g)\right)$. Let us choose $h \in \mathrm{GL}_{n}\left(k_{E}\right)$ such that $h^{-1 t} g^{-1} h=g^{-1}$. So, $\chi_{\tau_{\theta}}(g)=\chi_{\tau_{\theta}}\left(g^{-1}\right)=\chi_{\tau_{\theta}}\left(h^{-1 t} g^{-1} h\right)=\chi_{\tau_{\theta}}\left({ }^{t} g^{-1}\right)$. Let us denote $\tau_{\theta}^{\eta}(g)$ for $\tau_{\theta}(\eta(g))$ where $\eta: g \longrightarrow{ }^{t} g^{-1}$ is a group automorphism of $\mathrm{GL}_{n}\left(k_{E}\right)$. Hence $\chi_{\tau_{\theta}^{\eta}}(g)=\chi_{\tau_{\theta}}\left({ }^{t} g^{-1}\right)$. But we have already shown before that $\chi_{\tau_{\theta}}\left({ }^{t} g^{-1}\right)=\chi_{\tau_{\theta}^{\vee}}(g)$. so $\chi_{\tau_{\theta}^{\vee}}(g)=\chi_{\tau_{\theta}^{\eta}}(g)$. This implies $\tau_{\theta}^{\vee} \simeq \tau_{\theta}^{\eta}$. Hence $\tau_{\theta}^{\iota}=\tau_{\theta}^{\eta} \simeq$ $\tau_{\theta}^{\vee} \simeq \tau_{\theta^{-1}}$ (since $\left.\tau_{\theta}^{\vee} \simeq \tau_{\theta}^{\eta}, \tau_{\theta}^{\vee} \simeq \tau_{\theta^{-1}}\right)$. Now from the hypothesis of Propn. we know that $\tau_{\theta}^{\iota} \simeq \tau_{\theta}$, so this implies $\tau_{\theta} \simeq \tau_{\theta^{-1}}$ ( since $\tau_{\theta}^{\iota} \simeq \tau_{\theta^{-1}}$ ). But as $\theta$ is a regular character $\theta^{\gamma}=\theta^{-1}$ for some $\gamma \in \Gamma=\operatorname{Gal}\left(l / k_{E}\right)$ where $\left[l: k_{E}\right]=n$.

$\Longleftarrow$ Now we can reverse the arguments and show that if $\theta^{\gamma}=\theta^{-1}$ for some $\gamma \in \Gamma=\operatorname{Gal}\left(l / k_{E}\right)$ then $\tau_{\theta}^{\iota} \simeq \tau_{\theta^{-1}}$. 
Proposition 3.9. If $\theta$ is a regular character of $l^{\times}$such that $\theta^{\gamma}=\theta^{-1}$ for some $\gamma \in \Gamma$ then $n$ is even. Conversely, if $n=2 m$ is even and $\theta$ is a regular character of $l^{\times}$then $\theta^{\Phi^{m}}=\theta^{-1}$.

Proof. $\Longrightarrow$ Suppose $\theta$ is a regular character of $l^{\times}$such that $\theta^{\gamma}=\theta^{-1}$ for some $\gamma \in \Gamma$. We know that $\Gamma=<\Phi>$ where $\Phi: l \longrightarrow l$ is the Frobenius map given by $\Phi(\lambda)=\lambda^{q}$ for $\lambda \in l$. Now $\Phi^{n}(\lambda)=\lambda^{q^{n}}=\lambda$ for $\lambda \in l \Longrightarrow \Phi^{n}=1$. So for $\lambda \in l^{\times}$we have $\theta^{\gamma^{2}}(\lambda)=\theta^{\gamma}(\gamma(\lambda))=\theta^{-1}(\gamma(\lambda))=\theta\left((\gamma(\lambda))^{-1}\right)=\theta\left(\gamma\left(\lambda^{-1}\right)\right)=$ $\left.\theta^{\gamma}(\lambda)^{-1}=\theta^{-1}\left(\lambda^{-1}\right)=\theta\left((\lambda)^{-1}\right)^{-1}\right)=\theta(\lambda)$. So this implies $\theta^{\gamma^{2}}=\theta$. As $\theta$ is a regular character, so we have $\gamma^{2}=1$. Now for $\lambda \in l^{\times}$we have $\theta^{\Phi}(\lambda)=$ $\theta(\Phi(\lambda))=\theta\left(\lambda^{q}\right)=\theta^{q}(\lambda)$. That implies $\theta^{\Phi}=\theta^{q}$. As $\gamma^{2}=1 \Longrightarrow \gamma=1$ or $\gamma$ has order 2. If $\gamma=1$ as $\theta^{\gamma}=\theta^{-1} \Longrightarrow \theta=\theta^{-1} \Longrightarrow \theta(\lambda)=\theta^{-1}(\lambda)$ for $\lambda \in l^{\times} \Longrightarrow \theta(\lambda)=\theta\left(\lambda^{-1}\right) \Longrightarrow \theta\left(\lambda^{2}\right)=1 \Longrightarrow(\theta(\lambda))^{2}=1 \Longrightarrow \theta(\lambda)=\{ \pm 1\}$ for $\lambda \in l^{\times}$.

Let $q$ be an odd prime power. So for $\lambda \in l^{\times}$we have $\theta^{\Phi}(\lambda)=\theta^{q}(\lambda)=(\theta(\lambda))^{q}=$ $\theta(\lambda)$ (since $\theta(\lambda)=\{ \pm 1\}$ ). So this implies $\theta^{\Phi}=\theta$ and that further implies $\Phi=1$ as $\theta$ is a regular character $\Longrightarrow n=1$ which contradicts our assumption that cardinality of $\Gamma$ is greater than 1 . Now suppose $q$ is a prime power of 2 . As the characteristic of $k_{E}=2$ that implies $+1=-1$ in $k_{E}$. So $\theta(\lambda)= \pm 1=1$ for $\lambda \in l^{\times}$. So we have for $\lambda \in l^{\times}, \theta^{\Phi}(\lambda)=\theta^{q}(\lambda)=(\theta(\lambda))^{q}=1($ since $\theta(\lambda)=1)$. And this implies $\theta^{\Phi}=\theta$ and that further implies $\Phi=1$ as $\theta$ is a regular character $\Longrightarrow n=1$ which contradicts our assumption that cardinality of $\Gamma$ is greater than 1.

Hence $\gamma^{2}=1$ or $\gamma$ has order 2 , since $\gamma \neq 1$. Now $\Gamma$ has order $n$ and $\gamma \in \Gamma$ has order 2. So $2 \mid n \Longrightarrow n$ is even.

$\Longleftarrow$ Suppose $n$ is even. Let $n=2 m$ where $m \in \mathbb{N}$. Now 


$$
\operatorname{Hom}\left(l^{\times}, \mathbb{C}^{\times}\right) \cong l^{\times}
$$

So $\operatorname{Hom}\left(l^{\times}, \mathbb{C}^{\times}\right)$is a cyclic group of order $\left(q^{n}-1\right)=\left(q^{2 m}-1\right)$. Hence for every divisor $d$ of $\left(q^{2 m}-1\right)$, there exists an element in $\operatorname{Hom}\left(l^{\times}, \mathbb{C}^{\times}\right)$of order $d$. As $\left(q^{m}+1\right)$ is a divisor of $\left(q^{2 m}-1\right)$, hence there exists an element $\theta$ in $\operatorname{Hom}\left(l^{\times}, \mathbb{C}^{\times}\right)$ of order $\left(q^{m}+1\right)$. So $\theta^{q^{m}+1}=1 \Longrightarrow \theta^{q^{m}}=\theta^{-1} \Longrightarrow \theta^{\Phi^{m}}=\theta^{-1}\left(\right.$ since $\left.\theta^{\Phi}=\theta^{q}\right)$. Hence we have $\theta^{\gamma}=\theta^{-1}$, where $\gamma=\Phi^{m}$.

Now we claim that the character $\theta$ is regular. Suppose $\theta^{\gamma}=\theta$ for some $\gamma \in \Gamma$. Let $\gamma=\Phi^{k}$ for some $k \in \mathbb{Z}$. Then we have $\theta^{\Phi^{k}}=\theta \Longrightarrow \theta^{q^{k}}=\theta \Longrightarrow \theta^{q^{k}-1}=1$. As $\theta$ has order $\left(q^{m}+1\right)$ that means $\left(q^{m}+1\right) \mid\left(q^{k}-1\right)$. By Euclidean Algorithm, we have $k=m d+r$ where $r, d \in \mathbb{Z}, 0 \leqslant r<m$. If $d=0$ then $k=r<m$ which contradicts the fact that $\left(q^{m}+1\right) \mid\left(q^{k}-1\right)$. So $d \geqslant 1$. Now as $\left(q^{m}+1\right) \mid$ $\left(q^{k}-1\right) \Longrightarrow\left(q^{m}+1\right)\left|\left(\left(q^{k}-1\right)+\left(q^{m}+1\right)\right) \Longrightarrow\left(q^{m}+1\right)\right|\left(q^{k}+q^{m}\right) \Longrightarrow\left(q^{m}+1\right) \mid$ $\left(q^{m d+r}+q^{m}\right) \Longrightarrow\left(q^{m}+1\right) \mid q^{m}\left(q^{m(d-1)+r}+1\right)$. But as $q^{m}$ and $\left(q^{m}+1\right)$ are relatively prime, so this implies $\left(q^{m}+1\right) \mid\left(q^{m(d-1)+r}+1\right)$. But $\left(q^{m}+1\right) \mid\left(q^{m(d-1)+r}+1\right) \Longrightarrow$ $\left(q^{m}+1\right)\left|\left(\left(q^{m(d-1)+r}+1\right)-\left(q^{m}+1\right)\right) \Longrightarrow\left(q^{m}+1\right)\right| q^{m}\left(q^{m(d-2)+r}-1\right)$. But as $q^{m}$ and $\left(q^{m}+1\right)$ are relatively prime, so this implies $\left(q^{m}+1\right) \mid\left(q^{m(d-2)+r}-1\right)$. Continuing the above process, we have $\left(q^{m}+1\right) \mid\left(q^{r}+1\right)$ if $d$ is odd and $\left(q^{m}+1\right) \mid\left(q^{r}-1\right)$ if $d$ is even. As $r<m$, the above conditions are possible only when $r=0$. If $r=0$, then $k=m d$. So if $d$ is odd then $\left(q^{m}+1\right) \mid 2 \Longrightarrow\left(q^{m}+1\right)$ is either 1 or 2 . If $\left(q^{m}+1\right)=1$ then $q=0$ which is a contradiction. So let $\left(q^{m}+1\right)=2$ then we have $q=1$ which is again a contradiction as $q$ is a prime power. So $d$ has to be even. Let $d$ be even and is greater than 2 . So $d$ can take values $4,6,8, \ldots$. But as $k=m d$, so $k$ can take values $4 m, 6 m, 8 m, \ldots$ That is $k$ can take values $2 n, 3 n, 6 n, \ldots$ which is a contradiction as $k<n$. So $d=2$. Hence $k=2 m=n$. So $\Phi^{k}=\Phi^{n}=1 \Longrightarrow \gamma=1$. So $\theta$ is a regular character. 
Combining Propn. 3.8 and Propn. 3.9 we have the following Propn.

Proposition 3.10. Let $\theta$ be a regular character of $l^{\times}$. Then $\tau_{\theta}^{\iota} \simeq \tau_{\theta} \Longleftrightarrow n$ is even.

We know that $\rho_{0}$ is an irreducible cuspidal representation of $K$. But $K \cong \mathfrak{P}_{0}$. So $\rho_{0}$ can be viewed as a representation of $\mathfrak{P}_{0}$. Now let us compute $N_{G}\left(\rho_{0}\right)$, where $N_{G}\left(\rho_{0}\right)=\left\{m \in N_{G}\left(\mathfrak{P}_{0}\right) \mid \rho_{0} \simeq \rho_{0}^{m}\right\}$. Let $m \in N_{G}\left(\mathfrak{P}_{0}\right)$. Hence $m$ is either $J$ or $m$ is of the form $\left[\begin{array}{cc}z k & 0 \\ 0 & t^{(z k)}-1\end{array}\right]$ for some $z \in Z, k \in K$.

Proposition 3.11. If $m=\left[\begin{array}{cc}z k & 0 \\ 0 & t \overline{(z k)}-1\end{array}\right]$ for some $z \in Z, k \in K$ then $\rho_{0}{ }^{m} \simeq \rho_{0}$.

Proof. As $\rho_{0}$ is an irreducible cuspidal representation of $K$, so $K$ normalizes $\rho_{0}$. Clearly, $Z$ normalizes $\rho_{0}$. Thus $Z K$ normalizes $\rho_{0}$. As $\rho_{0}$ can also be viewed as a representation of $\mathfrak{P}_{0}$, so $\rho_{0}{ }^{m} \simeq \rho_{0}$ where $m=\left[\begin{array}{cc}z k & 0 \\ 0 & t \overline{(z k)}-1\end{array}\right]$ for some $z \in Z, k \in K$

Proposition 3.12. If $m=J=\left[\begin{array}{ll}0 & 1 \\ 1 & 0\end{array}\right]$ then $\rho_{0}{ }^{m} \simeq \rho_{0}$ only when $n$ is even.

Proof. We know that $\iota: a \longrightarrow{ }^{t} \bar{a}^{-1}$ is a group homomorphism of $\mathrm{GL}_{n}\left(k_{E}\right)$. Now $\iota: a \longrightarrow{ }^{t} \bar{a}^{-1}$ can be inflated to a group homomorphism of $\mathrm{GL}_{n}\left(\mathfrak{O}_{E}\right)$. Further, $\iota$ can be viewed as a group homomorphism from $\mathfrak{P}_{0}$ to $\mathfrak{P}_{0}$ given by:

$$
\iota\left(\left[\begin{array}{cc}
a & 0 \\
0 & { }^{t} \bar{a}^{-1}
\end{array}\right]\right)=\left[\begin{array}{cc}
{ }^{t} \bar{a}^{-1} & 0 \\
0 & a
\end{array}\right]
$$


where $a \in \mathrm{GL}_{n}\left(\mathfrak{O}_{E}\right)$. Let $g=\left[\begin{array}{cc}a & 0 \\ 0 & { }^{t} \bar{a}^{-1}\end{array}\right]$. If $m=J$ then $\rho_{0}{ }^{m}(g)=\rho_{0}\left(J g J^{-1}\right)=$ $\rho_{0}\left(\left[\begin{array}{cc}{ }^{t} \bar{a}^{-1} & 0 \\ 0 & a\end{array}\right]\right)=\rho_{0}(\iota(g))=\rho_{0}^{\iota}(g)$. So $\rho_{0}{ }^{m}(g)=\rho_{0}^{\iota}(g)$ for all $g \in \mathfrak{P}_{0} \Longrightarrow \rho_{0}{ }^{m}=$ $\rho_{0}^{\iota}$. But from the hypothesis of the Propn. we know that $\rho_{0}^{m} \simeq \rho_{0}$. So we have $\rho_{0} \simeq \rho_{0}^{\iota}$. Now from Propn. 3.10, $\rho_{0} \simeq \rho_{0}^{\iota}=\rho_{0}^{m} \Longleftrightarrow n$ is even.

So we have the following conclusion about $N_{G}\left(\rho_{0}\right)$ for ramified case: If $n$ is odd then $N_{G}\left(\rho_{0}\right)=Z(L) \mathfrak{P}_{0}$ and if $n$ is even then $N_{G}\left(\rho_{0}\right)=Z(L) \mathfrak{P}_{0} \rtimes\langle J\rangle$.

Lemma 3.13. When $n$ is odd in the unramified case or when $n$ is even in the ramified case, we have $N_{G}\left(\rho_{0}\right)=\left\langle\mathfrak{P}_{0}, w_{0}, w_{1}\right\rangle$, where $w_{0}=J$ and $w_{1}=$ $\left[\begin{array}{cc}0 & \bar{\varpi}_{E}^{-1} 1 \\ \varpi_{E} 1 & 0\end{array}\right]$

Proof. Let $\zeta=w_{0} w_{1}$. So $\zeta=\left[\begin{array}{cc}\varpi_{E} 1 & 0 \\ 0 & \bar{\varpi}_{E}^{-1} 1\end{array}\right]$. We can clearly see that $w_{0}^{2}=1$. So $w_{0}=w_{0}^{-1}$ and $w_{1}=w_{0}^{-1} \zeta=w_{0} \zeta$. From the hypothesis of lemma, we have $N_{G}\left(\rho_{0}\right)=Z(L) \mathfrak{P}_{0} \rtimes\langle J\rangle$. As any element in $E^{\times}$can be written as $u \varpi^{n}$ for some $n \in \mathbb{Z}, u \in \mathfrak{O}_{E}^{\times}$, so $Z(L)=Z\left(\mathfrak{P}_{0}\right)\langle\zeta\rangle$. So $Z(L) \mathfrak{P}_{0}=\left\langle\mathfrak{P}_{0}, \zeta\right\rangle$. Hence $N_{G}\left(\rho_{0}\right)=\left\langle\mathfrak{P}_{0}, \zeta\right\rangle \rtimes J$. But $J=w_{0}, w_{1}=w_{0} \zeta$. So $N_{G}\left(\rho_{0}\right)=\left\langle\mathfrak{P}_{0}, w_{0}, w_{1}\right\rangle$.

\subsection{Structure of $\mathcal{H}(G, \rho)$ : unramified case}

In this section we determine the structure of $\mathcal{H}(G, \rho)$ for the unramified case when $n$ is odd. Using cuspidality of $\rho_{0}$, it can be shown by Theorem 4.15 in [8], that $\mathfrak{I}_{G}(\rho)=\mathfrak{P} N_{G}\left(\rho_{0}\right) \mathfrak{P}$. But from lemma $3.13, N_{G}\left(\rho_{0}\right)=\left\langle\mathfrak{P}_{0}, w_{0}, w_{1}\right\rangle$. So $\mathfrak{I}_{G}(\rho)=$ $\mathfrak{P}\left\langle\mathfrak{P}_{0}, w_{0}, w_{1}\right\rangle \mathfrak{P}=\mathfrak{P}\left\langle w_{0}, w_{1}\right\rangle \mathfrak{P}$, as $\mathfrak{P}_{0}$ is a subgroup of $\mathfrak{P}$. Let $V$ be the vector 
space corresponding to $\rho$. Let us recall that $\mathcal{H}(G, \rho)$ consists of maps $f: G \rightarrow$ $\operatorname{End}_{\mathbb{C}}\left(V^{\vee}\right)$ such that support of $f$ is compact and $f\left(p g p^{\prime}\right)=\rho^{\vee}(p) f(g) \rho^{\vee}\left(p^{\prime}\right)$ for $p, p^{\prime} \in \mathfrak{P}, g \in G$. In fact $\mathcal{H}(G, \rho)$ consists of $\mathbb{C}$-linear combinations of maps $f: G \longrightarrow \operatorname{End}_{\mathbb{C}}\left(V^{\vee}\right)$ such that $f$ is supported on $\mathfrak{P} x \mathfrak{P}$ where $x \in \mathfrak{I}_{G}(\rho)$ and $f\left(p x p^{\prime}\right)=\rho^{\vee}(p) f(x) \rho^{\vee}\left(p^{\prime}\right)$ for $p, p^{\prime} \in \mathfrak{P}$. We shall now show there exists $\phi_{0} \in$ $\mathcal{H}(G, \rho)$ with support $\mathfrak{P} w_{0} \mathfrak{P}$ and satisfies $\phi_{0}^{2}=q^{n}+\left(q^{n}-1\right) \phi_{0}$. Let

$$
\begin{gathered}
K(0)=\mathrm{U}(n, n) \cap \mathrm{GL}_{2 n}\left(\mathfrak{O}_{E}\right)=\left\{\left.g \in \mathrm{GL}_{2 n}\left(\mathfrak{O}_{E}\right)\right|^{t} \bar{g} J g=J\right\}, \\
K_{1}(0)=\left\{g \in 1+\left.\varpi_{E} \mathrm{M}_{2 n}\left(\mathfrak{O}_{E}\right)\right|^{t} \bar{g} J g=J\right\}, \\
\mathrm{G}=\left\{\left.g \in \mathrm{GL}_{2 n}\left(k_{E}\right)\right|^{t} \bar{g} J g=J\right\} .
\end{gathered}
$$

The map $r$ from $K(0)$ to $\mathrm{G}$ given by $r: K(0) \stackrel{\bmod p_{E}}{\longrightarrow} \mathrm{G}$ is a surjective group homomorphism with kernel $K_{1}(0)$. So by the first isomorphism theorem of groups we have:

$$
\begin{aligned}
& \frac{K(0)}{K_{1}(0)} \cong \mathrm{G} . \\
& r(\mathfrak{P})=\mathrm{P}=\left[\begin{array}{cc}
\mathrm{GL}_{n}\left(k_{E}\right) & \mathrm{M}_{n}\left(k_{E}\right) \\
0 & \mathrm{GL}_{n}\left(k_{E}\right)
\end{array}\right] \cap \mathrm{G}=\text { Siegel parabolic subgroup of } \mathrm{G} .
\end{aligned}
$$

Now $P=L \ltimes U$, where $L$ is the Siegel Levi component of $P$ and $U$ is the unipotent radical of G. Here

$$
\begin{gathered}
\mathrm{L}=\left\{\left[\begin{array}{cc}
a & 0 \\
0 & { }^{t} \bar{a}^{-1}
\end{array}\right] \mid a \in \mathrm{GL}_{n}\left(k_{E}\right)\right\}, \\
\mathbf{U}=\left\{\left[\begin{array}{cc}
1 & X \\
0 & 1
\end{array}\right] \mid X \in \mathrm{M}_{n}\left(k_{E}\right), X+{ }^{t} \bar{X}=0\right\} .
\end{gathered}
$$


Let $V$ be the vector space corresponding to $\rho$. The Hecke algebra $\mathcal{H}(K(0), \rho)$ is a sub-algebra of $\mathcal{H}(G, \rho)$.

Let $\bar{\rho}$ be the representation of $\mathrm{P}$ which when inflated to $\mathfrak{P}$ is given by $\rho$ and $V$ is also the vector space corresponding to $\bar{\rho}$. The Hecke algebra $\mathcal{H}(\mathrm{G}, \bar{\rho})$ looks as follows:

$$
\mathcal{H}(\mathrm{G}, \bar{\rho})=\left\{\begin{array}{l|l}
f: \mathrm{G} \rightarrow \operatorname{End}_{\mathbb{C}}\left(V^{\vee}\right) & \begin{array}{l}
f\left(p g p^{\prime}\right)=\bar{\rho}^{\vee}(p) f(g) \bar{\rho}^{\vee}\left(p^{\prime}\right) \\
\text { where } p, p^{\prime} \in \mathrm{P}, g \in \mathrm{G}
\end{array}
\end{array}\right\} .
$$

Now the homomorphism $r: K(0) \longrightarrow \mathrm{G}$ extends to a map from $\mathcal{H}(K(0), \rho)$ to $\mathcal{H}(\mathrm{G}, \bar{\rho})$ which we again denote by $r$. Thus $r: \mathcal{H}(K(0), \rho) \longrightarrow \mathcal{H}(\mathrm{G}, \bar{\rho})$ is given by

$$
\begin{gathered}
r(\phi)(r(x))=\phi(x) \\
\text { for } \phi \in \mathcal{H}(K(0), \rho) \text { and } x \in K(0) .
\end{gathered}
$$

Proposition 3.14. The map $r: \mathcal{H}(K(0), \rho) \longrightarrow \mathcal{H}(\mathrm{G}, \bar{\rho})$ is an algebra isomorphism.

Proof. To prove that the map $r$ is an isomorphism of algebras, we have to show that $r$ is a homomorphism of algebras and is a bijective map.

In order to show that the map $r$ is a homomorphism, we need to show that it is $\mathbb{C}$-linear and it preserves convolution. It is obvious that the map $r$ is $\mathbb{C}$-linear. Let us now show that the map preserves convolution.

If $x \in K(0)$ and $\phi_{1}, \phi_{2} \in \mathcal{H}(K(0), \rho)$ then

$$
\left(\phi_{1} * \phi_{2}\right)(x)=\int_{K(0)} \phi_{1}(y) \phi_{2}\left(y^{-1} x\right) d y .
$$


Now

$$
\int_{K(0)} \phi_{1}(y) \phi_{2}\left(y^{-1} x\right) d y=\sum_{y \in \mathfrak{P} / K(0)} \phi_{1}\left(x y^{-1}\right) \phi_{2}(y) .
$$

Hence

$$
\begin{aligned}
r\left(\phi_{1} * \phi_{2}\right)(r(x)) & =\left(\phi_{1} * \phi_{2}\right)(x) \\
& =\sum_{y \in \mathfrak{P} / K(0)} \phi_{1}\left(x y^{-1}\right) \phi_{2}(y) \\
& =\sum_{y \in \mathfrak{P} / K(0)}\left(r\left(\phi_{1}\right)\left(r\left(x y^{-1}\right)\right)\right)\left(r\left(\phi_{2}\right)(r(y))\right) \\
& =\sum_{r(y) \in \mathrm{P} / \mathrm{G}}\left(r\left(\phi_{1}\right)\left(r(x)(r(y))^{-1}\right)\right)\left(r\left(\phi_{2}\right)(r(y))\right) \\
& =\left(r\left(\phi_{1}\right) * r\left(\phi_{2}\right)\right)(r(x)) .
\end{aligned}
$$

So we have $r\left(\phi_{1} * \phi_{2}\right)(r(x))=\left(r\left(\phi_{1}\right) * r\left(\phi_{2}\right)\right)(r(x))$. But $r$ is a surjective group homomorphism from $K(0)$ to $\mathrm{G}$. Hence $r\left(\phi_{1} * \phi_{2}\right)(y)=\left(r\left(\phi_{1}\right) * r\left(\phi_{2}\right)\right)(y)$ for $y \in \mathrm{G}$ which would imply that $r\left(\phi_{1} * \phi_{2}\right)=\left(r\left(\phi_{1}\right) * r\left(\phi_{2}\right)\right)$. Hence $r$ is a homomorphism of algebras.

In order to show that $r$ is bijective map, we first show here that the map $r$ is a one-one map. Let $\phi_{1}, \phi_{2} \in \mathcal{H}(K(0), \rho), y \in \mathrm{G}$. Suppose $r\left(\phi_{1}\right)(y)=r\left(\phi_{2}\right)(y)$. As $r$ is surjective map from $K(0)$ to $\mathrm{G}$, so there exists $x \in K(0)$ such that $r(x)=y$. So $r\left(\phi_{1}\right)(r(x))=r\left(\phi_{2}\right)(r(x)) \Longrightarrow \phi_{1}(x)=\phi_{2}(x)$. As $r$ is a surjective map from $K(0)$ to $\mathrm{G}$, so when $y$ spans over $\mathrm{G}, x$ spans over $K(0)$. So $\phi_{1}(x)=\phi_{2}(x)$ for $x \in K(0) \Longrightarrow \phi_{1}=\phi_{2}$. So $r$ is a one-one map.

Now we show that $r$ is a surjective map from $\mathcal{H}(K(0), \rho)$ to $\mathcal{H}(\mathrm{G}, \bar{\rho})$. Let $\psi \in \mathcal{H}(\mathrm{G}, \bar{\rho})$, then $\psi: \mathrm{G} \longrightarrow \operatorname{End}_{\mathbb{C}} V$ is a map such that $\psi\left(p g p^{\prime}\right)=\psi(g)$ for $p, p^{\prime} \in$ $\mathrm{P}, g \in \mathrm{G}$. As $r$ is a surjective map from $K(0)$ to $\mathrm{G}$, so $\psi \circ r$ makes sense. Now let 
us call $\psi \circ r$ as $\phi$. So $\phi$ is a map from $K(0)$ to $E n d_{\mathbb{C}} V$. Let $p, p^{\prime} \in \mathfrak{P}, k \in K(0)$, so $\phi\left(p k p^{\prime}\right)=(\psi \circ r)\left(p k p^{\prime}\right)=\psi\left(r\left(p k p^{\prime}\right)\right)=\psi\left(r(p) r(k) r\left(p^{\prime}\right)\right)=\psi(r(k))=(\psi \circ r)(k)=$ $\phi(k)$. So $\phi \in \mathcal{H}(K(0), \rho)$. Let $y \in \mathrm{G}$. So there exits $x \in K(0)$ such that $r(x)=y$. Now consider $\psi(y)=\psi(r(x))=(\psi \circ r)(x)=\phi(x)=r(\phi)(r(x))=r(\phi)(y)$. So $\psi(y)=r(\phi)(y)$ for $y \in \mathrm{G} \Longrightarrow \psi=r(\phi)$. Hence $r$ is a surjective map.

As $r$ is both one-one and surjective map, hence it is a bijective map.

Let $w=r\left(w_{0}\right)=r\left(\left[\begin{array}{ll}0 & 1 \\ 1 & 0\end{array}\right]\right)=\left[\begin{array}{ll}0 & 1 \\ 1 & 0\end{array}\right] \in \mathrm{G}$. Clearly $K(0) \supseteq \mathfrak{P} \amalg \mathfrak{P} w_{0} \mathfrak{P} \Longrightarrow$
$r(K(0)) \supseteq r\left(\mathfrak{P} \amalg \mathfrak{P} w_{0} \mathfrak{P}\right) \Longrightarrow \mathrm{G} \supseteq r(\mathfrak{P}) \amalg r\left(\mathfrak{P} w_{0} \mathfrak{P}\right)=\mathrm{P} \amalg \mathrm{P} w \mathrm{P}$. So G $\supseteq \mathrm{P} \amalg \mathrm{P} w \mathrm{P}$.

Now $\operatorname{Ind} d_{\mathrm{P}}^{\mathrm{G}} \bar{\rho}=\pi_{1} \oplus \pi_{2}$, where $\pi_{1}, \pi_{2}$ are distinct irreducible representations of $\mathrm{G}$ with $\operatorname{dim} \pi_{2} \geqslant \operatorname{dim} \pi_{1}$. Let $\lambda=\frac{\operatorname{dim} \pi_{2}}{\operatorname{dim} \pi_{1}}$. By Propn. 3.2 in [4], there exists a unique $\phi$ in $\mathcal{H}(\mathrm{G}, \bar{\rho})$ with support $\mathrm{P} w \mathrm{P}$ such that $\phi^{2}=\lambda+(\lambda-1) \phi$. By Propn. 3.14, there is a unique element $\phi_{0}$ in $\mathcal{H}(K(0), \rho)$ such that $r\left(\phi_{0}\right)=\phi$. Thus $\operatorname{supp}\left(\phi_{0}\right)=\mathfrak{P} w_{0} \mathfrak{P}$ and $\phi_{0}^{2}=\lambda+(\lambda-1) \phi_{0}$. From Lemma 3.12 in $[7], \lambda=q^{n}$. Hence $\phi_{0}^{2}=q^{n}+\left(q^{n}-1\right) \phi_{0}$. As support of $\phi_{0}=\mathfrak{P} w_{0} \mathfrak{P} \subseteq K(0) \subseteq G$, so $\phi_{0}$ can be extended to $G$ and viewed as an element of $\mathcal{H}(G, \rho)$. Thus $\phi_{0}$ satisfies the following relation in $\mathcal{H}(G, \rho)$ :

$$
\phi_{0}^{2}=q^{n}+\left(q^{n}-1\right) \phi_{0}
$$

We shall now show there exists $\phi_{1} \in \mathcal{H}(G, \rho)$ with support $\mathfrak{P} w_{1} \mathfrak{P}$ satisfying the same relation as $\phi_{0}$. Let $\eta=\left[\begin{array}{cc}0 & 1 \\ \varpi_{E} 1 & 0\end{array}\right]$. Now we can check that $\eta w_{0} \eta^{-1}=w_{1}$. Recall that $\mathfrak{P}$ looks as follows: 


$$
\mathfrak{P}=\left[\begin{array}{cc}
\mathrm{GL}_{n}\left(\mathfrak{O}_{E}\right) & \mathrm{M}_{n}\left(\mathfrak{O}_{E}\right) \\
\mathrm{M}_{n}\left(p_{E}\right) & \mathrm{GL}_{n}\left(\mathfrak{O}_{E}\right)
\end{array}\right] \cap G
$$

Lemma 3.15. $\eta \mathfrak{P} \eta^{-1}=\mathfrak{P}$.

Proof.

$$
\begin{aligned}
\mathfrak{P} & =\left[\begin{array}{cc}
\mathrm{GL}_{n}\left(\mathfrak{O}_{E}\right) & \mathrm{M}_{n}\left(\mathfrak{O}_{E}\right) \\
\mathrm{M}_{n}\left(p_{E}\right) & \mathrm{GL}_{n}\left(\mathfrak{O}_{E}\right)
\end{array}\right] \cap G \\
\Longrightarrow \eta \mathfrak{P} \eta^{-1} & =\eta\left[\begin{array}{cc}
\mathrm{GL}_{n}\left(\mathfrak{O}_{E}\right) & \mathrm{M}_{n}\left(\mathfrak{O}_{E}\right) \\
\mathrm{M}_{n}\left(p_{E}\right) & \mathrm{GL}_{n}\left(\mathfrak{O}_{E}\right)
\end{array}\right] \eta^{-1} \bigcap \eta G \eta^{-1} .
\end{aligned}
$$

It is easy to show that

$$
\eta\left[\begin{array}{cc}
\mathrm{GL}_{n}\left(\mathfrak{O}_{E}\right) & \mathrm{M}_{n}\left(\mathfrak{O}_{E}\right) \\
\mathrm{M}_{n}\left(p_{E}\right) & \mathrm{GL}_{n}\left(\mathfrak{O}_{E}\right)
\end{array}\right] \eta^{-1}=\left[\begin{array}{cc}
\mathrm{GL}_{n}\left(\mathfrak{O}_{E}\right) & \mathrm{M}_{n}\left(\mathfrak{O}_{E}\right) \\
\mathrm{M}_{n}\left(p_{E}\right) & \mathrm{GL}_{n}\left(\mathfrak{O}_{E}\right)
\end{array}\right]
$$

Now we claim that $\eta G \eta^{-1}=G$. To prove this let us consider

$$
G^{\prime}=\left\{\left.g \in \mathrm{GL}_{2 n}(E)\right|^{t} \bar{g} J g=\lambda(g) J \text { for some } \lambda(g) \in F^{\times}\right\} .
$$

Now $\eta \in G^{\prime}$ clearly, as ${ }^{t} \bar{\eta} J \eta=\varpi_{E} J=\varpi_{F} J$. And $\lambda: G^{\prime} \longrightarrow F^{\times}$is a homomorphism of groups with kernel $G$. So $G \unlhd G^{\prime}$. As $\eta \in G^{\prime}$ and $G \unlhd G^{\prime}$, so $\eta G \eta^{-1}=G$. Hence $\eta \mathfrak{P} \eta^{-1}=\mathfrak{P}$.

As $\mathfrak{P} \subseteq K(0)$ and $w_{0} \in K(0)$, so $K(0) \supseteq \mathfrak{P} \amalg \mathfrak{P} w_{0} \mathfrak{P} \Longrightarrow \eta K(0) \eta^{-1} \supseteq \eta \mathfrak{P} \eta^{-1} \amalg$ $\eta \mathfrak{P} w_{0} \mathfrak{P} \eta^{-1}$. But from lemma 3.15 , we know that $\eta \mathfrak{P} \eta^{-1}=\mathfrak{P}$ and $\eta \mathfrak{P} w_{0} \mathfrak{P} \eta^{-1}=$ $\left(\eta \mathfrak{P} \eta^{-1}\right)\left(\eta w_{0} \eta^{-1}\right)\left(\eta \mathfrak{P} \eta^{-1}\right)=\mathfrak{P} w_{1} \mathfrak{P}\left(\right.$ since $\left.\eta w_{0} \eta^{-1}=w_{1}\right)$. So $\eta K(0) \eta^{-1} \supseteq \mathfrak{P} \amalg$ $\mathfrak{P} w_{1} \mathfrak{P}$. 
Let $r^{\prime}$ be homomorphism of groups given by the map $r^{\prime}: \eta k(0) \eta^{-1} \longrightarrow \mathrm{G}$ such that $r^{\prime}(x)=\left(\eta^{-1} x \eta\right) \bmod p_{E}$ for $x \in K(0)$. Observe that $r^{\prime}$ is a surjective homomorphism of groups because $r^{\prime}\left(\eta K(0) \eta^{-1}\right)=\left(\eta^{-1} \eta K(0) \eta^{-1} \eta\right) \bmod p_{E}=$ $K(0) \bmod p_{E}=\mathrm{G}$. The kernel of group homomorphism is $\eta K_{1}(0) \eta^{-1}$. Now by the first isomorphism theorem of groups we have $\frac{\eta K(0) \eta^{-1}}{\eta K_{1}(0) \eta^{-1}} \cong \frac{K(0)}{K_{1}(0)} \cong \mathrm{G}$. Also $r^{\prime}\left(\eta \mathfrak{P} \eta^{-1}\right)=\left(\eta^{-1} \eta \mathfrak{P} \eta^{-1} \eta\right) \bmod p_{E}=\mathfrak{P} \bmod p_{E}=\mathrm{P}$. Let $\bar{\rho}$ be representation of $\mathrm{P}$ which when inflated to $\mathfrak{P}$ is given by $\rho$. The Hecke algebra of $\eta K(0) \eta^{-1}$ which we denote by $\mathcal{H}\left(\eta K(0) \eta^{-1}, \rho\right)$ is a sub-algebra of $\mathcal{H}(G, \rho)$.

The map $r^{\prime}: \eta K(0) \eta^{-1} \longrightarrow \mathrm{G}$ extends to a map from $\mathcal{H}\left(\eta K(0) \eta^{-1}, \rho\right)$ to $\mathcal{H}(\mathrm{G}, \bar{\rho})$ which we gain denote by $r^{\prime}$. Thus $r^{\prime}: \mathcal{H}\left(\eta K(0) \eta^{-1}, \rho\right) \longrightarrow \mathcal{H}(\mathrm{G}, \bar{\rho})$ is given by

$$
\begin{gathered}
r^{\prime}\left(\phi\left(r^{\prime}(x)\right)=\phi(x)\right. \\
\text { for } \phi \in \mathcal{H}\left(\eta K(0) \eta^{-1}, \rho\right) \text { and } x \in \eta K(0) \eta^{-1} .
\end{gathered}
$$

The proof that $r^{\prime}$ is an isomorphism goes in the similar lines as Propn. 3.14 .We can observe that $r^{\prime}\left(w_{1}\right)=w \in \mathrm{G}$, where $w$ is defined as before in this section. As we know from our previous discussion in this section, that there exists a unique $\phi$ in $\mathcal{H}(\mathrm{G}, \bar{\rho})$ with support $\mathrm{P} w \mathrm{P}$ such that $\phi^{2}=q^{n}+\left(q^{n}-1\right) \phi$. Hence there is a unique element $\phi_{1} \in \mathcal{H}\left(\eta K(0) \eta^{-1}, \rho\right)$ such that $r^{\prime}\left(\phi_{1}\right)=\phi$. Thus $\operatorname{supp}\left(\phi_{1}\right)=\mathfrak{P} w_{1} \mathfrak{P}$ and $\phi_{1}^{2}=q^{n}+\left(q^{n}-1\right) \phi_{1}$. Now $\phi_{1}$ can be extended to $G$ and viewed as an element in $\mathcal{H}(G, \rho)$ as $\mathfrak{P} w_{1} \mathfrak{P} \subseteq \eta K(0) \eta^{-1} \subseteq G$. Thus $\phi_{1}$ satisfies the following relation in $\mathcal{H}(G, \rho)$ :

$$
\phi_{1}^{2}=q^{n}+\left(q^{n}-1\right) \phi_{1}
$$

Thus we have shown there exists $\phi_{i} \in \mathcal{H}(G, \rho)$ with $\operatorname{supp}\left(\phi_{i}\right)=\mathfrak{P} w_{i} \mathfrak{P}$ satisfying 
$\phi_{i}^{2}=q^{n}+\left(q^{n}-1\right) \phi_{i}$ for $i=0,1$. It can be further shown that $\phi_{0}$ and $\phi_{1}$ generate the Hecke algebra $\mathcal{H}(G, \rho)$. Let us denote the Hecke algebra $\mathcal{H}(G, \rho)$ by $\mathcal{A}$. So

$$
\mathcal{A}=\mathcal{H}(G, \rho)=\left\langle\begin{array}{l|l}
\phi_{i}: G \rightarrow E n d_{\mathbb{C}}\left(\rho^{\vee}\right) & \begin{array}{l}
\phi_{i} \text { is supported on } \mathfrak{P} w_{i} \mathfrak{P} \\
\text { and } \left.\phi_{i}\left(p w_{i} p^{\prime}\right)=\rho^{\vee}(p) \phi_{i}\left(w_{i}\right) \rho^{\vee}\left(p^{\prime}\right)\right\rangle \\
\text { where } p, p^{\prime} \in \mathfrak{P}, g \in G, i=0,1
\end{array}
\end{array}\right.
$$

where $\phi_{i}$ satisfies the relation:

$$
\phi_{i}^{2}=q^{n}+\left(q^{n}-1\right) \phi_{i} \text { for } i=0,1 .
$$

Lemma 3.16. $\phi_{0}$ and $\phi_{1}$ are units in $\mathcal{A}$.

Proof. As $\phi_{i}^{2}=q^{n}+\left(q^{n}-1\right) \phi_{i}$ for $i=0,1$. So $\phi_{i}\left(\frac{\phi_{i}+\left(1-q^{n}\right) 1}{q^{n}}\right)=1$ for $\mathrm{i}=0,1$. Hence $\phi_{0}$ and $\phi_{1}$ are units in $\mathcal{A}$.

Lemma 3.17. Let $\phi, \psi \in \mathcal{H}(G, \rho)$ with support of $\phi, \psi$ being $\mathfrak{P} x \mathfrak{P}, \mathfrak{P} y \mathfrak{P}$ respectively. Then $\operatorname{supp}(\phi * \psi)=\operatorname{supp}(\phi \psi) \subseteq(\operatorname{supp}(\phi))(\operatorname{supp}(\psi))=\mathfrak{P} x \mathfrak{P} y \mathfrak{P}$.

Proof. As $\operatorname{supp}(\phi)=\mathfrak{P} x \mathfrak{P}$ and $\operatorname{supp}(\psi)=\mathfrak{P} y \mathfrak{P}$, so if $z \in \operatorname{supp}(\phi * \psi)$ then $(\phi *$ $\psi(z)=\int_{G} \phi\left(z r^{-1}\right) \psi(r) d r \neq 0$. So there exists $r \in G$ such that $\phi\left(z r^{-1}\right) \psi(r) \neq 0$. Because if $\phi\left(z r^{-1}\right) \psi(r)=0$ for $r \in G$ then $\int_{G} \phi\left(z r^{-1}\right) \psi(r)=0 \Longrightarrow(\phi * \psi)(z)=0$ which is a contradiction. So $\phi\left(z r^{-1}\right) \psi(r) \neq 0$ for some $r \in G$. As $\phi\left(z r^{-1}\right) \neq 0 \Longrightarrow$ $\operatorname{supp}(\phi)=\mathfrak{P} x \mathfrak{P}$ and $\psi(r) \neq 0 \Longrightarrow r \in \operatorname{supp}(\psi)=\mathfrak{P} y \mathfrak{P}$. Hence $\left(z r^{-1}\right)(r)=z \in$ $(\operatorname{supp}(\phi))(\operatorname{supp}(\psi))=(\mathfrak{P} x \mathfrak{P})(\mathfrak{P} y \mathfrak{P})=\mathfrak{P} x \mathfrak{P} y \mathfrak{P}$. Hence $\operatorname{supp}(\phi * \psi)=\operatorname{supp}(\phi \psi)$ $\subseteq(\operatorname{supp}(\phi))(\operatorname{supp}(\psi))=\mathfrak{P} x \mathfrak{P} y \mathfrak{P}$.

From B-N pair structure theory we can show that, $\mathfrak{P} x \mathfrak{P} y \mathfrak{P}=\mathfrak{P} x y \mathfrak{P} \Longleftrightarrow$ $l(x y)=l(x)+l(y)$. From lemma 3.17, we have $\operatorname{supp}\left(\phi_{0} \phi_{1}\right) \subseteq \mathfrak{P} w_{0} \mathfrak{P} w_{1} \mathfrak{P}$. But $\mathfrak{P} w_{0} \mathfrak{P} w_{1} \mathfrak{P}=\mathfrak{P} w_{0} w_{1} \mathfrak{P}\left(\right.$ since $\left.l\left(w_{0} w_{1}\right)=l\left(w_{0}\right)+l\left(w_{1}\right)\right) . \quad$ Thus $\operatorname{supp}\left(\phi_{0} \phi_{1}\right) \subseteq$ $\mathfrak{P} w_{0} w_{1} \mathfrak{P}$. Let $\zeta=w_{0} w_{1}$, So 


$$
\zeta=\left[\begin{array}{cc}
\varpi 1 & 0 \\
0 & \varpi^{-1} 1
\end{array}\right] .
$$

As $\phi_{0}, \phi_{1}$ are units in algebra $\mathcal{A}$, so $\psi=\phi_{0} \phi_{1}$ is a unit too in $\mathcal{A}$ and $\psi^{-1}=\phi_{1}^{-1} \phi_{0}^{-1}$. Now as we have seen before that $\operatorname{supp}\left(\phi_{0} \phi_{1}\right) \subseteq \mathfrak{P} w_{0} w_{1} \mathfrak{P} \Longrightarrow$ $\operatorname{supp}(\psi) \subseteq \mathfrak{P} \zeta \mathfrak{P} \Longrightarrow \operatorname{supp}(\psi)=\varnothing$ or $\mathfrak{P} \zeta \mathfrak{P}$. If $\operatorname{supp}(\psi)=\varnothing \Longrightarrow \psi=0$ which is a contradiction as $\psi$ is a unit in $\mathcal{A}$. $\operatorname{So} \operatorname{supp}(\psi)=\mathfrak{P} \zeta \mathfrak{P}$. As $\psi$ is a unit in $\mathcal{A}$, we can show as before from B-N pair structure theory that $\operatorname{supp}\left(\psi^{2}\right)=\mathfrak{P} \zeta^{2} \mathfrak{P}$. Hence by induction on $n \in \mathbb{N}$, we can further show from B-N pair structure theory that $\operatorname{supp}\left(\psi^{n}\right)=\mathfrak{P} \zeta^{n} \mathfrak{P}$ for $n \in \mathbb{N}$.

Now $\mathcal{A}$ contains a sub- algebra generated by $\psi, \psi^{-1}$ over $\mathbb{C}$ and we denote this sub-algebra by $\mathcal{B}$. So $\mathcal{B}=\mathbb{C}\left[\psi, \psi^{-1}\right]$ where

$$
\mathcal{B}=\mathbb{C}\left[\psi, \psi^{-1}\right]=\left\{\begin{array}{l|l}
c_{k} \psi^{k}+\cdots+c_{l} \psi^{l} & \begin{array}{l}
c_{k}, \ldots, c_{l} \in \mathbb{C} ; \\
k<l ; k, l \in \mathbb{Z}
\end{array}
\end{array}\right\} .
$$

Proposition 3.18. The unique algebra homomorphism $\mathbb{C}\left[x, x^{-1}\right] \longrightarrow \mathcal{B}$ given by $x \longrightarrow \psi$ is an isomorphism. So $\mathcal{B} \simeq \mathbb{C}\left[x, x^{-1}\right]$.

Proof. It is obvious that the map is an algebra homomorphism and is surjective as $\left\{\psi^{n} \mid n \in \mathbb{Z}\right\}$ spans $\mathcal{B}$. Now we show that the kernel of map is 0. Suppose $c_{k} \psi^{k}+\cdots+c_{l} \psi^{l}=0$ with $c_{k}, \ldots, c_{l} \in \mathbb{C} ; l>k \geqslant 0 ; l, k \in \mathbb{Z}$. Let $x \in \operatorname{supp} \psi^{s}=$ $\mathfrak{P} \zeta^{s} \mathfrak{P}$ where $0 \leqslant k \leqslant s \leqslant l$. As double cosets of a group are disjoint or equal, so $\psi^{s}(x) \neq 0$ and $\psi^{i}(x)=0$ for $0 \leqslant k \leqslant i \leqslant l, i \neq s$. Hence $c_{k} \psi^{k}(x)+\cdots+c_{l} \psi^{l}(x)=0$ would imply that $c_{s}=0$. In a similar way we can show that $c_{k}=c_{k+1}=\ldots=c_{l}=$ 0 . So $\left\{\psi^{k}, \psi^{k+1}, \ldots, \psi^{l}\right\}$ is a linearly independent set when $0 \leqslant k<l ; k, l \in \mathbb{Z}$. Now suppose if $k<0$ and let $c_{k} \psi^{k}+\cdots+c_{l} \psi^{l}=0$ with $c_{k}, \ldots, c_{l} \in \mathbb{C} ; k, l \in \mathbb{Z}$. Let us assume without loss of generality that $k<l$. Multiplying throughout the above expression by $\psi^{-k}$, we have $c_{k}+\cdots+c_{l} \psi^{l-k}=0$. Now repeating the 
previous argument we have $c_{k}=c_{k+1}=\ldots=c_{l}=0$. So again $\left\{\psi^{k}, \psi^{k+1}, \ldots, \psi^{l}\right\}$ is a linearly independent set when $k<0 ; k<l ; k, l \in \mathbb{Z}$. Hence $\mathcal{B} \simeq \mathbb{C}\left[x, x^{-1}\right]$.

\subsection{Structure of $\mathcal{H}(G, \rho)$ : ramified case}

In this section we determine the structure of $\mathcal{H}(G, \rho)$ for the ramified case when $n$ is even. Recall $\mathfrak{I}_{G}(\rho)=\mathfrak{P} N_{G}\left(\rho_{0}\right) \mathfrak{P}$. But from lemma 3.13, $N_{G}\left(\rho_{0}\right)=\left\langle\mathfrak{P}_{0}, w_{0}, w_{1}\right\rangle$. So $\mathfrak{I}_{G}(\rho)=\mathfrak{P}\left\langle\mathfrak{P}_{0}, w_{0}, w_{1}\right\rangle \mathfrak{P}=\mathfrak{P}\left\langle w_{0}, w_{1}\right\rangle \mathfrak{P}$, as $\mathfrak{P}_{0}$ is a subgroup of $\mathfrak{P}$. Let $V$ be the vector space corresponding to $\rho$. Let us recall that $\mathcal{H}(G, \rho)$ consists of maps $f: G \rightarrow \operatorname{End}_{\mathbb{C}}\left(V^{\vee}\right)$ such that support of $f$ is compact and $f\left(p g p^{\prime}\right)=$ $\rho^{\vee}(p) f(g) \rho^{\vee}\left(p^{\prime}\right)$ for $p, p^{\prime} \in \mathfrak{P}, g \in G$. In fact $\mathcal{H}(G, \rho)$ consists of $\mathbb{C}$-linear combinations of maps $f: G \longrightarrow \operatorname{End}_{\mathbb{C}}\left(V^{\vee}\right)$ such that $f$ is supported on $\mathfrak{P} x \mathfrak{P}$ where $x \in \mathfrak{I}_{G}(\rho)$ and $f\left(p x p^{\prime}\right)=\rho^{\vee}(p) f(x) \rho^{\vee}\left(p^{\prime}\right)$ for $p, p^{\prime} \in \mathfrak{P}$. We shall now show there exists $\phi_{0} \in \mathcal{H}(G, \rho)$ with support $\mathfrak{P} w_{0} \mathfrak{P}$ and satisfies $\phi_{0}^{2}=q^{n / 2}+\left(q^{n / 2}-1\right) \phi_{0}$. Let

$$
\begin{gathered}
K(0)=\mathrm{U}(n, n) \cap \mathrm{GL}_{2 n}\left(\mathfrak{O}_{E}\right)=\left\{\left.g \in \mathrm{GL}_{2 n}\left(\mathfrak{O}_{E}\right)\right|^{t} \bar{g} J g=J\right\}, \\
K_{1}(0)=\left\{g \in 1+\left.\varpi_{E} \mathrm{M}_{2 n}\left(\mathfrak{O}_{E}\right)\right|^{t} \bar{g} J g=J\right\}, \\
\mathrm{G}=\left\{\left.g \in \mathrm{GL}_{2 n}\left(k_{E}\right)\right|^{t} \bar{g} J g=J\right\} .
\end{gathered}
$$

The map $r$ from $K(0)$ to $\mathrm{G}$ given by $r: K(0) \stackrel{\bmod p_{E}}{\longrightarrow} \mathrm{G}$ is a surjective group homomorphism with kernel $K_{1}(0)$. So by the first isomorphism theorem of groups we have:

$$
\frac{K(0)}{K_{1}(0)} \cong \mathrm{G}
$$




$$
r(\mathfrak{P})=\mathrm{P}=\left[\begin{array}{cc}
\mathrm{GL}_{n}\left(k_{E}\right) & \mathrm{M}_{n}\left(k_{E}\right) \\
0 & \mathrm{GL}_{n}\left(k_{E}\right)
\end{array}\right] \cap \mathrm{G}=\text { Siegel parabolic subgroup of } \mathrm{G} .
$$

Now $P=L \ltimes U$, where $L$ is the Siegel Levi component of $P$ and $U$ is the unipotent radical of G. Here

$$
\begin{gathered}
\mathrm{L}=\left\{\left[\begin{array}{cc}
a & 0 \\
0 & { }^{t} \bar{a}^{-1}
\end{array}\right] \mid a \in \mathrm{GL}_{n}\left(k_{E}\right)\right\}, \\
\mathrm{U}=\left\{\left[\begin{array}{cc}
1 & X \\
0 & 1
\end{array}\right] \mid X \in \mathrm{M}_{n}\left(k_{E}\right), X+{ }^{t} \bar{X}=0\right\} .
\end{gathered}
$$

Let $V$ be the vector space corresponding to $\rho$. The Hecke algebra $\mathcal{H}(K(0), \rho)$ is a sub-algebra of $\mathcal{H}(G, \rho)$.

Let $\bar{\rho}$ be the representation of $\mathrm{P}$ which when inflated to $\mathfrak{P}$ is given by $\rho$ and $V$ is also the vector space corresponding to $\bar{\rho}$. The Hecke algebra $\mathcal{H}(\mathrm{G}, \bar{\rho})$ looks as follows:

$$
\mathcal{H}(\mathrm{G}, \bar{\rho})=\left\{\begin{array}{l|l}
f: \mathrm{G} \rightarrow \operatorname{End}_{\mathbb{C}}\left(V^{\vee}\right) & \begin{array}{l}
f\left(p g p^{\prime}\right)=\bar{\rho}^{\vee}(p) f(g) \bar{\rho}^{\vee}\left(p^{\prime}\right) \\
\text { where } p, p^{\prime} \in \mathrm{P}, g \in \mathrm{G}
\end{array}
\end{array}\right\}
$$

Now the homomorphism $r: K(0) \longrightarrow G$ extends to a map from $\mathcal{H}(K(0), \rho)$ to $\mathcal{H}(\mathrm{G}, \bar{\rho})$ which we again denote by $r$. Thus $r: \mathcal{H}(K(0), \rho) \longrightarrow \mathcal{H}(\mathrm{G}, \bar{\rho})$ is given by

$$
\begin{gathered}
r(\phi)(r(x))=\phi(x) \\
\text { for } \phi \in \mathcal{H}(K(0), \rho) \text { and } x \in K(0) .
\end{gathered}
$$

As in the unramified case, when $n$ is odd, we can show that $\mathcal{H}(K(0), \rho)$ is 
isomorphic to $\mathcal{H}(\mathrm{G}, \bar{\rho})$ as algebras via $r$.

Let $w=r\left(w_{0}\right)=r\left(\left[\begin{array}{ll}0 & 1 \\ 1 & 0\end{array}\right]\right)=\left[\begin{array}{ll}0 & 1 \\ 1 & 0\end{array}\right] \in \mathrm{G}$. Clearly $K(0) \supseteq \mathfrak{P} \amalg \mathfrak{P} w_{0} \mathfrak{P} \Longrightarrow$
$r(K(0)) \supseteq r\left(\mathfrak{P} \amalg \mathfrak{P} w_{0} \mathfrak{P}\right) \Longrightarrow \mathrm{G} \supseteq r(\mathfrak{P}) \amalg r\left(\mathfrak{P} w_{0} \mathfrak{P}\right)=\mathrm{P} \amalg \mathrm{P} w \mathrm{P}$. So G $\supseteq \mathrm{P} \amalg \mathrm{P} w \mathrm{P}$.

Now $\mathrm{G}$ is a finite group. In fact, it is the special orthogonal group consisting of matrices of size $2 n \times 2 n$ over finite field $k_{E}$ or $\mathbb{F}_{q}$. So $G=S O_{2 n}\left(\mathbb{F}_{q}\right)$.

According to the Theorem 6.3 in [4], there exists a unique $\phi$ in $\mathcal{H}(\mathrm{G}, \bar{\rho})$ with support $\mathrm{P} w \mathrm{P}$ such that $\phi^{2}=q^{n / 2}+\left(q^{n / 2}-1\right) \phi$. Hence there is a unique element $\phi_{0} \in \mathcal{H}(K(0), \rho)$ such that $r\left(\phi_{0}\right)=\phi$. Thus $\operatorname{supp}\left(\phi_{0}\right)=\mathfrak{P} w_{0} \mathfrak{P}$ and $\phi_{0}^{2}=q^{n / 2}+$ $\left(q^{n / 2}-1\right) \phi_{0}$. Now $\phi_{0}$ can be extended to $G$ and viewed as an element in $\mathcal{H}(G, \rho)$ as $\mathfrak{P} w_{0} \mathfrak{P} \subseteq K(0) \subseteq G$. Thus $\phi_{0}$ satisfies the following relation in $\mathcal{H}(G, \rho)$ :

$$
\phi_{0}^{2}=q^{n / 2}+\left(q^{n / 2}-1\right) \phi_{0}
$$

We shall now show there exists $\phi_{1} \in \mathcal{H}(G, \rho)$ with support $\mathfrak{P} w_{1} \mathfrak{P}$ satisfying the same relation as $\phi_{0}$.

We know that $w_{1}=\left[\begin{array}{cc}0 & \bar{\varpi}_{E}^{-1} 1 \\ \varpi_{E} 1 & 0\end{array}\right], \bar{\varpi}_{E}^{-1}=-\varpi_{E}$. So $w_{1}=\left[\begin{array}{cc}0 & -\varpi_{E}^{-1} 1 \\ \varpi_{E} 1 & 0\end{array}\right]$. Let $\eta=\left[\begin{array}{cc}\varpi_{E} 1 & 0 \\ 0 & 1\end{array}\right]$. So, $\eta w_{1} \eta^{-1}=J^{\prime}=\left[\begin{array}{cc}0 & -1 \\ 1 & 0\end{array}\right]$. Recall that $\mathfrak{P}$ looks as follows:

$$
\mathfrak{P}=\left[\begin{array}{cc}
\mathrm{GL}_{n}\left(\mathfrak{O}_{E}\right) & \mathrm{M}_{n}\left(\mathfrak{O}_{E}\right) \\
\mathrm{M}_{n}\left(p_{E}\right) & \mathrm{GL}_{n}\left(\mathfrak{O}_{E}\right)
\end{array}\right] \cap G .
$$

Now

$$
\eta\left[\begin{array}{cc}
\mathrm{GL}_{n}\left(\mathfrak{O}_{E}\right) & \mathrm{M}_{n}\left(\mathfrak{O}_{E}\right) \\
\mathrm{M}_{n}\left(p_{E}\right) & \mathrm{GL}_{n}\left(\mathfrak{O}_{E}\right)
\end{array}\right] \eta^{-1}=\left[\begin{array}{cc}
\mathrm{GL}_{n}\left(\mathfrak{O}_{E}\right) & \mathrm{M}_{n}\left(p_{E}\right) \\
\mathrm{M}_{n}\left(\mathfrak{O}_{E}\right) & \mathrm{GL}_{n}\left(\mathfrak{O}_{E}\right)
\end{array}\right]
$$




$$
\eta G \eta^{-1}=G^{\prime}=\left\{\left.g \in \mathrm{GL}_{2 n}(E)\right|^{t} \bar{g} J^{\prime} g=J^{\prime}\right\}
$$

Hence

$$
\eta \mathfrak{P} \eta^{-1}=\left[\begin{array}{cc}
\mathrm{GL}_{n}\left(\mathfrak{O}_{E}\right) & \mathrm{M}_{n}\left(p_{E}\right) \\
\mathrm{M}_{n}\left(\mathfrak{O}_{E}\right) & \mathrm{GL}_{n}\left(\mathfrak{O}_{E}\right)
\end{array}\right] \cap G^{\prime}
$$

Therefore $\eta \mathfrak{P} \eta^{-1}$ is the opposite of the Siegel Parahoric subgroup of $G^{\prime}$. Let

$$
K^{\prime}(0)=\left\langle\mathfrak{P}, w_{1}\right\rangle .
$$

And let

$$
\begin{aligned}
\mathrm{G}^{\prime} & =\left\{\left.g \in \mathrm{GL}_{2 n}\left(k_{E}\right)\right|^{t} \bar{g} J^{\prime} g=J^{\prime}\right\} \\
& =\left\{\left.g \in \mathrm{GL}_{2 n}\left(k_{E}\right)\right|^{t} g J^{\prime} g=J^{\prime}\right\} .
\end{aligned}
$$

Let $r^{\prime}: K^{\prime}(0) \longrightarrow \mathrm{G}^{\prime}$ be the group homomorphism given by

$$
r^{\prime}(x)=\left(\eta x \eta^{-1}\right) \bmod p_{E} \text { where } x \in K^{\prime}(0) .
$$

So we have $r^{\prime}(K(0))=\left(\eta K^{\prime}(0) \eta^{-1}\right) \bmod p_{E}=\left(\eta\left\langle\mathfrak{P}, w_{1}\right\rangle \eta^{-1}\right) \bmod p_{E}$. Let $r^{\prime}(\mathfrak{P})=\left(\eta \mathfrak{P} \eta^{-1}\right) \bmod p_{E}=\overline{\mathrm{P}}^{\prime}$. We can see that $r^{\prime}\left(w_{1}\right)=\left(\eta w_{1} \eta^{-1}\right) \bmod p_{E}=$ $J^{\prime} \bmod p_{E}=w^{\prime}=\left[\begin{array}{cc}0 & -1 \\ 1 & 0\end{array}\right]$.

$$
\text { So } \overline{\mathrm{P}}^{\prime}=r^{\prime}(\mathfrak{P})=\left(\eta \mathfrak{P} \eta^{-1}\right) \bmod p_{E}=\left[\begin{array}{cc}
\mathrm{GL}_{n}\left(k_{E}\right) & 0 \\
\mathrm{M}_{n}\left(k_{E}\right) & \mathrm{GL}_{n}\left(k_{E}\right)
\end{array}\right] \cap \mathrm{G}^{\prime} \text {. Clearly } \overline{\mathrm{P}}^{\prime} \text { is }
$$

the opposite of Siegel parabolic subgroup of $\mathrm{G}^{\prime}$. Hence $r^{\prime}(K(0))=\left\langle\overline{\mathrm{P}}^{\prime}, w^{\prime}\right\rangle=\mathrm{G}^{\prime}$, as $\overline{\mathrm{P}}^{\prime}$ is a maximal subgroup of $\mathrm{G}^{\prime}$ and $w^{\prime}$ does not lie in $\overline{\mathrm{P}}^{\prime}$. So $r^{\prime}$ is a surjective homomorphism of groups. 
Let $V$ be the vector space corresponding to $\rho$. The Hecke algebra $\mathcal{H}\left(K^{\prime}(0), \rho\right)$ is a sub-algebra of $\mathcal{H}(G, \rho)$.

Let $\bar{\rho}^{\prime}$ be the representation of $\overline{\mathrm{P}}^{\prime}$ which when inflated to ${ }^{\eta} \mathfrak{P}$ is given by ${ }^{\eta} \rho$ and $V$ is also the vector space corresponding to $\bar{\rho}^{\prime}$. Now the Hecke algebra $\mathcal{H}\left(\mathrm{G}^{\prime}, \bar{\rho}^{\prime}\right)$ looks as follows:

$$
\mathcal{H}\left(\mathrm{G}^{\prime}, \bar{\rho}^{\prime}\right)=\left\{\begin{array}{l|l}
f: \mathrm{G}^{\prime} \rightarrow \operatorname{End}_{\mathbb{C}}\left(V^{\vee}\right) & \begin{array}{l}
f\left(p g p^{\prime}\right)=\bar{\rho}^{\vee}(p) f(g) \bar{\rho}^{\prime \vee}\left(p^{\prime}\right) \\
\text { where } p, p^{\prime} \in \overline{\mathrm{P}}^{\prime}, g \in \mathrm{G}^{\prime}
\end{array}
\end{array}\right\} .
$$

Now the homomorphism $r^{\prime}: K^{\prime}(0) \longrightarrow \mathrm{G}^{\prime}$ extends to a map from $\mathcal{H}\left(K^{\prime}(0), \rho\right)$ to $\mathcal{H}\left(\mathrm{G}^{\prime}, \bar{\rho}^{\prime}\right)$ which we again denote by $r^{\prime}$. Thus $r^{\prime}: \mathcal{H}\left(K^{\prime}(0), \rho\right) \longrightarrow \mathcal{H}\left(\mathrm{G}^{\prime}, \bar{\rho}^{\prime}\right)$ is given by

$$
r^{\prime}(\phi)\left(r^{\prime}(x)\right)=\phi(x)
$$

for $\phi \in \mathcal{H}\left(K^{\prime}(0), \rho\right)$ and $x \in K^{\prime}(0)$.

As in the unramified case when $n$ is odd, we can show that $\mathcal{H}\left(K^{\prime}(0), \rho\right)$ is isomorphic to $\mathcal{H}\left(\mathrm{G}^{\prime}, \bar{\rho}^{\prime}\right)$ as algebras via $r^{\prime}$.

Clearly $K^{\prime}(0) \supseteq \mathfrak{P} \amalg \mathfrak{P} w_{1} \mathfrak{P} \Longrightarrow r^{\prime}\left(K^{\prime}(0)\right) \supseteq r^{\prime}\left(\mathfrak{P} \amalg \mathfrak{P} w_{1} \mathfrak{P}\right) \Longrightarrow \mathrm{G}^{\prime} \supseteq r^{\prime}(\mathfrak{P}) \amalg$ $r^{\prime}\left(\mathfrak{P} w_{1} \mathfrak{P}\right)=\overline{\mathrm{P}}^{\prime} \amalg \overline{\mathrm{P}}^{\prime} w^{\prime} \overline{\mathrm{P}}^{\prime}$. So $\mathrm{G}^{\prime} \supseteq \overline{\mathrm{P}}^{\prime} \amalg \overline{\mathrm{P}}^{\prime} w^{\prime} \overline{\mathrm{P}}^{\prime}$.

Now $G^{\prime}$ is a finite group. In fact, it is the symplectic group consisting of matrices of size $2 n \times 2 n$ over finite field $k_{E}$ or $\mathbb{F}_{q}$. So $\mathrm{G}^{\prime}=S p_{2 n}\left(\mathbb{F}_{q}\right)$.

According to the Theorem 6.3 in [4], there exists a unique $\phi$ in $\mathcal{H}\left(\mathrm{G}^{\prime}, \bar{\rho}^{\prime}\right)$ with support $\overline{\mathrm{P}}^{\prime} w^{\prime} \overline{\mathrm{P}}^{\prime}$ such that $\phi^{2}=q^{n / 2}+\left(q^{n / 2}-1\right) \phi$. Hence there is a unique element $\phi_{1} \in \mathcal{H}\left(K^{\prime}(0), \rho\right)$ such that $r^{\prime}\left(\phi_{1}\right)=\phi$. Thus $\operatorname{supp}\left(\phi_{1}\right)=\mathfrak{P} w_{1} \mathfrak{P}$ and $\phi_{1}^{2}=q^{n / 2}+\left(q^{n / 2}-1\right) \phi_{1}$. Now $\phi_{1}$ can be extended to $G$ and viewed as an element 
in $\mathcal{H}(G, \rho)$ as $\mathfrak{P} w_{1} \mathfrak{P} \subseteq K^{\prime}(0) \subseteq G$. Thus $\phi_{1}$ satisfies the following relation in $\mathcal{H}(G, \rho)$ :

$$
\phi_{1}^{2}=q^{n / 2}+\left(q^{n / 2}-1\right) \phi_{1}
$$

Thus we have shown there exists $\phi_{i} \in \mathcal{H}(G, \rho)$ with $\operatorname{supp}\left(\phi_{i}\right)=\mathfrak{P} w_{i} \mathfrak{P}$ satisfying $\phi_{i}^{2}=q^{n / 2}+\left(q^{n / 2}-1\right) \phi_{i}$ for $i=0,1$. It can be further shown that $\phi_{0}$ and $\phi_{1}$ generate the Hecke algebra $\mathcal{H}(G, \rho)$. Let us denote the Hecke algebra $\mathcal{H}(G, \rho)$ by $\mathcal{A}$. So

$$
\mathcal{A}=\mathcal{H}(G, \rho)=\left\langle\phi_{i}: G \rightarrow \operatorname{End}_{\mathbb{C}}\left(\rho^{\vee}\right) \mid \begin{array}{l}
\phi_{i} \text { is supported on } \mathfrak{P} w_{i} \mathfrak{P} \\
\operatorname{and} \phi_{i}\left(p w_{i} p^{\prime}\right)=\rho^{\vee}(p) \phi_{i}\left(w_{i}\right) \rho^{\vee}\left(p^{\prime}\right) \\
\text { where } p, p^{\prime} \in \mathfrak{P}, g \in G, i=0,1
\end{array}\right\rangle
$$

where $\phi_{i}$ has support $\mathfrak{P} w_{i} \mathfrak{P}$ and $\phi_{i}$ satisfies the relation:

$$
\phi_{i}^{2}=q^{n / 2}+\left(q^{n / 2}-1\right) \phi_{i} \text { for } i=0,1
$$

Lemma 3.19. $\phi_{0}$ and $\phi_{1}$ are units in $\mathcal{A}$.

Proof. As $\phi_{i}^{2}=q^{n / 2}+\left(q^{n / 2}-1\right) \phi_{i}$ for $i=0,1$. So $\phi_{i}\left(\frac{\phi_{i}+\left(1-q^{n / 2}\right) 1}{q^{n / 2}}\right)=1$ for $\mathrm{i}=0,1$. Hence $\phi_{0}$ and $\phi_{1}$ are units in $\mathcal{A}$.

As $\phi_{0}, \phi_{1}$ are units in $\mathcal{A}$ which is an algebra, so $\psi=\phi_{0} \phi_{1}$ is a unit too in $\mathcal{A}$ and $\psi^{-1}=\phi_{1}^{-1} \phi_{0}^{-1}$. As in the unramified case when $n$ is odd, we can show that $\mathcal{A}$ contains sub-algebra $\mathcal{B}=\mathbb{C}\left[\psi, \psi^{-1}\right]$ where

$$
\mathcal{B}=\mathbb{C}\left[\psi, \psi^{-1}\right]=\left\{\begin{array}{l|l}
c_{k} \psi^{k}+\cdots+c_{l} \psi^{l} & \begin{array}{l}
c_{k}, \ldots, c_{l} \in \mathbb{C} ; \\
k<l ; k, l \in \mathbb{Z}
\end{array}
\end{array}\right\}
$$


Further, as in the unramified case when $n$ is odd, we can show that $\mathbb{C}\left[\psi, \psi^{-1}\right] \simeq$ $\mathbb{C}\left[x, x^{-1}\right]$ as $\mathbb{C}$-algebras.

\subsection{Structure of $\mathcal{H}\left(L, \rho_{0}\right)$}

In this section we describe the structure of $\mathcal{H}\left(L, \rho_{0}\right)$. Thus we need first to determine

$$
N_{L}\left(\rho_{0}\right)=\left\{m \in N_{L}\left(\mathfrak{P}_{0}\right) \mid \rho_{0}^{m} \simeq \rho_{0}\right\}
$$

We know from lemma 3.1 that $N_{\mathrm{GL}_{n}(E)}\left(K_{0}\right)=K_{0} Z$, so we have $N_{L}\left(\mathfrak{P}_{0}\right)=$ $Z(L) \mathfrak{P}_{0}$. Since $Z(L)$ clearly normalizes $\rho_{0}$ and $\rho_{0}$ is an irreducible cuspidal representation of $\mathfrak{P}_{0}$, so $N_{L}\left(\rho_{0}\right)=Z(L) \mathfrak{P}_{0}$.

Now that we have calculated $N_{L}\left(\rho_{0}\right)$, we determine the structure of $\mathcal{H}\left(L, \rho_{0}\right)$. Using the cuspidality of $\rho_{0}$, it can be shown by A.1 Appendix [8] that $\mathfrak{I}_{L}\left(\rho_{0}\right)=$ $\mathfrak{P}_{0} N_{L}\left(\rho_{0}\right) \mathfrak{P}_{0}$. As $N_{L}\left(\rho_{0}\right)=Z(L) \mathfrak{P}_{0}$, so $\mathfrak{I}_{L}\left(\rho_{0}\right)=\mathfrak{P}_{0} Z(L) \mathfrak{P}_{0} \mathfrak{P}_{0}=Z(L) \mathfrak{P}_{0}$. Let $V$ be the vector space of $\rho_{0}$.

The Hecke algebra $\mathcal{H}\left(L, \rho_{0}\right)$ consists of $\mathbb{C}$-linear combinations of maps $f: L \longrightarrow$ $\operatorname{End}_{\mathbb{C}}\left(V^{\vee}\right)$ such that each map $f$ is supported on $\mathfrak{P}_{0} x \mathfrak{P}_{0}$ where $x \in \mathfrak{I}_{L}\left(\rho_{0}\right)=$ $Z(L) \mathfrak{P}_{0}$ and $f\left(p x p^{\prime}\right)=\rho_{0}^{\vee}(p) f(x) \rho_{0}^{\vee}\left(p^{\prime}\right)$ for $p, p^{\prime} \in \mathfrak{P}_{0}$. It is clear that

$$
Z(L) \mathfrak{P}_{0}=\coprod_{n \in \mathbb{Z}} \mathfrak{P}_{0} \zeta^{n}
$$

So the Hecke algebra $\mathcal{H}\left(L, \rho_{0}\right)$ consists of $\mathbb{C}$-linear combinations of maps $f: L \longrightarrow \operatorname{End}_{\mathbb{C}}\left(V^{\vee}\right)$ such that each map $f$ is supported on $\mathfrak{P}_{0} x \mathfrak{P}_{0}$ where $x \in$ $\mathfrak{P}_{0} \zeta^{n}$ with $n \in \mathbb{Z}$ and $f\left(p x p^{\prime}\right)=\rho_{0}^{\vee}(p) f(x) \rho_{0}^{\vee}\left(p^{\prime}\right)$ for $p, p^{\prime} \in \mathfrak{P}_{0}$.

Let $\phi_{1}, \phi_{2} \in \mathcal{H}\left(L, \rho_{0}\right)$ with $\operatorname{supp}\left(\phi_{1}\right)=\mathfrak{P}_{0} z_{1}$ and $\operatorname{supp}\left(\phi_{2}\right)=\mathfrak{P}_{0} z_{2}$ respectively 
with $z_{1}, z_{2} \in Z(L)$. As $\rho_{0}$ is an irreducible cuspidal representation of $\mathfrak{P}_{0}$. So if $f \in \mathcal{H}\left(L, \rho_{0}\right)$ with $\operatorname{supp}(f)=\mathfrak{P}_{0} z$ where $z \in Z(L)$ then from Schur's lemma $f(z)=c 1_{V^{\vee}}$ for some $c \in \mathbb{C}^{\times}$. Hence $\phi_{1}\left(z_{1}\right)=c_{1} 1_{V^{\vee}}$ and $\phi_{2}\left(z_{2}\right)=c_{2} 1_{V^{\vee}}$ where $c_{1}, c_{2} \in \mathbb{C}^{\times}$.

We have $\operatorname{supp}\left(\phi_{1} \phi_{2}\right) \subseteq\left(\operatorname{supp}\left(\phi_{1}\right)\right)\left(\operatorname{supp}\left(\phi_{2}\right)\right)=\mathfrak{P}_{0} z_{1} \mathfrak{P}_{0} z_{2}=\mathfrak{P}_{0} z_{1} z_{2}$. The proof goes in the similar lines as lemma 3.17.

We assume without loss of generality that vol $\mathfrak{P}_{0}=\operatorname{volP}_{-}=\operatorname{volP}_{+}=1$. Thus we have volP $=1$.

Lemma 3.20. Let $\phi_{1}, \phi_{2} \in \mathcal{H}\left(L, \rho_{0}\right)$ with $\operatorname{supp}\left(\phi_{1}\right)=\mathfrak{P}_{0} z_{1}$ and $\operatorname{supp}\left(\phi_{2}\right)=\mathfrak{P}_{0} z_{2}$ where $z_{1}, z_{2} \in Z(L)$. Also let $\phi_{1}\left(z_{1}\right)=c_{1} 1_{V^{\vee}}$ and $\phi_{2}\left(z_{2}\right)=c_{2} 1_{V^{\vee}}$ where $c_{1}, c_{2} \in$ $\mathbb{C}^{\times}$. Then $\left(\phi_{1} * \phi_{2}\right)\left(z_{1} z_{2}\right)=\phi_{1}\left(z_{1}\right) \phi_{2}\left(z_{2}\right)=c_{1} c_{2} 1_{V^{\vee}}$.

Proof.

$$
\begin{aligned}
\left(\phi_{1} * \phi_{2}\right)\left(z_{1} z_{2}\right) & =\int_{L} \phi_{1}\left(z_{1} z_{2} y^{-1}\right) \phi_{2}(y) d y \\
& =\int_{\mathfrak{P}_{0}} \phi_{1}\left(z_{1} z_{2} z_{2}^{-1} p^{-1}\right) \phi_{2}\left(z_{2} p\right) d y \\
& =\int_{\mathfrak{P}_{0}} \phi_{1}\left(z_{1} p^{-1}\right) \phi_{2}\left(p z_{2}\right) d y \\
& =\int_{\mathfrak{P}_{0}} \phi_{1}\left(z_{1}\right) \rho_{0}^{\vee}\left(p^{-1}\right) \rho_{0}^{\vee}(p) \phi_{2}\left(z_{2}\right) d y \\
& =\int_{\mathfrak{P}_{0}} \phi_{1}\left(z_{1}\right) \phi_{2}\left(z_{2}\right) d y \\
& =\int_{\mathfrak{P}_{0}} c_{1} c_{2} 1_{V^{\vee}} d y \\
& =c_{1} c_{2} \operatorname{Vol}\left(\mathfrak{P}_{0}\right) 1_{V^{\vee}} \\
& =c_{1} c_{2} 1_{V^{\vee}} \\
& =\phi_{1}\left(z_{1}\right) \phi_{2}\left(z_{2}\right) .
\end{aligned}
$$


As $\operatorname{supp}\left(\phi_{1} * \phi_{2}\right)=\operatorname{supp}\left(\phi_{1} \phi_{2}\right) \subseteq \mathfrak{P}_{0} z_{1} z_{2}, \operatorname{so~} \operatorname{supp}\left(\phi_{1} * \phi_{2}\right)=\varnothing$ or $\mathfrak{P}_{0} z_{1} z_{2}$. If $\operatorname{supp}\left(\phi_{1} * \phi_{2}\right)=\varnothing$ then it means that $\left(\phi_{1} * \phi_{2}\right)=0$. This contradicts $\left(\phi_{1} *\right.$ $\left.\phi_{2}\right)\left(z_{1} z_{2}\right)=c_{1} c_{2} \neq 0$. So $\operatorname{supp}\left(\phi_{1} * \phi_{2}\right)=\mathfrak{P}_{0} z_{1} z_{2}$.

This implies that $\phi_{1}$ is invertible and $\phi_{1}^{-1}$ be it's inverse. Thus $\operatorname{supp}\left(\phi_{1}^{-1}\right)=$ $\mathfrak{P}_{0} z_{1}^{-1}$ and $\phi_{1}^{-1}\left(z_{1}^{-1}\right)=c_{1}^{-1} 1_{V^{\vee}}$.

Define $\alpha \in \mathcal{H}\left(L, \rho_{0}\right)$ by $\operatorname{supp}(\alpha)=\mathfrak{P}_{0} \zeta$ and $\alpha(\zeta)=1_{V^{\vee}}$.

Proposition 3.21. 1. $\alpha^{n}\left(\zeta^{n}\right)=(\alpha(\zeta))^{n}$ for $n \in \mathbb{Z}$.

2. $\operatorname{supp}\left(\alpha^{n}\right)=\mathfrak{P}_{0} \zeta^{n} \mathfrak{P}_{0}=\mathfrak{P}_{0} \zeta^{n}=\zeta^{n} \mathfrak{P}_{0}$ for $n \in \mathbb{Z}$.

Proof. As $\alpha: L \longrightarrow \operatorname{End}_{\mathbb{C}}\left(\rho_{0}^{\vee}\right)$, so $\alpha(\zeta) \in \operatorname{End}_{\mathbb{C}}\left(\rho_{0}^{\vee}\right)$. Now $\zeta \in Z(L), \mathfrak{P}_{0} \leqslant L$, so $\mathfrak{P}_{0}^{\zeta}=\mathfrak{P}_{0},\left(\rho_{0}^{\vee}\right)^{\zeta}=\rho_{0}^{\vee}$. We can see that $\zeta \in \mathfrak{I}_{L}\left(\rho_{0}^{\vee}\right)=\mathfrak{I}_{L}\left(\rho_{0}\right)=Z(L) \mathfrak{P}_{0}$, hence $\zeta$ intertwines $\rho_{0}^{\vee}$. Hence

$$
\begin{gathered}
\operatorname{Hom}_{\mathfrak{P}_{0} \cap \mathfrak{P}_{0}^{\zeta}}\left(\rho_{0}^{\vee},\left(\rho_{0}^{\vee}\right)^{\zeta}\right) \neq 0 \\
\Longrightarrow \operatorname{Hom}_{\mathfrak{P}_{0} \cap \mathfrak{F}_{0}}\left(\rho_{0}^{\vee}, \rho_{0}^{\vee}\right) \neq 0 \\
\Longrightarrow \operatorname{End}_{\mathfrak{P}_{0}}\left(\rho_{0}^{\vee}\right) \neq 0 .
\end{gathered}
$$

So $\alpha(\zeta) \in \operatorname{End}_{\mathfrak{P}_{0}}\left(\rho_{0}^{\vee}\right)$. As $\rho_{0}^{\vee}$ is an irreducible representation of $\mathfrak{P}_{0}$, so from Schur's lemma $\alpha(\zeta)$ is either zero or an isomorphism. But as $\alpha(\zeta) \neq 0 \Longrightarrow \alpha(\zeta)$ is an isomorphism $\Longrightarrow(\alpha(\zeta))^{-1}$ exists.

Using lemma 3.20 over and over we get, $\alpha^{n}\left(\zeta^{n}\right)=(\alpha(\zeta))^{n}$ for $n \in \mathbb{Z}$ and $\operatorname{supp}\left(\alpha^{n}\right)=\mathfrak{P}_{0} \zeta^{n} \mathfrak{P}_{0}=\mathfrak{P}_{0} \zeta^{n}=\zeta^{n} \mathfrak{P}_{0}$ for $n \in \mathbb{Z}$

We know that $\mathcal{H}\left(L, \rho_{0}\right)$ consists of $\mathbb{C}$-linear combinations of maps $f: L \longrightarrow$ $\operatorname{End}_{\mathbb{C}}\left(V^{\vee}\right)$ such that each map $f$ is supported on $\mathfrak{P}_{0} x \mathfrak{P}_{0}$ where $x \in \mathfrak{P}_{0} \zeta^{n}$ with $n \in \mathbb{Z}$ and $f\left(p x p^{\prime}\right)=\rho_{0}^{\vee}(p) f(x) \rho_{0}^{\vee}\left(p^{\prime}\right)$ for $p, p^{\prime} \in \mathfrak{P}_{0}$. So from Propn. 3.21, $\mathcal{H}\left(L, \rho_{0}\right)$ is generated as a $\mathbb{C}$-algebra by $\alpha$ and $\alpha^{-1}$. Hence $\mathcal{H}\left(L, \rho_{0}\right)=\mathbb{C}\left[\alpha, \alpha^{-1}\right]$. 
Proposition 3.22. The unique algebra homomorphism $\mathbb{C}\left[x, x^{-1}\right] \longrightarrow \mathbb{C}\left[\alpha, \alpha^{-1}\right]$ given by $x \longrightarrow \alpha$ is an isomorphism. So $\mathbb{C}\left[\alpha, \alpha^{-1}\right] \simeq \mathbb{C}\left[x, x^{-1}\right]$.

Proof. It is obvious that the map is an algebra homomorphism and is surjective as $\left\{\alpha^{n} \mid n \in \mathbb{Z}\right\}$ spans $\mathbb{C}\left[\alpha, \alpha^{-1}\right]$. Now we show that the kernel of map is 0 . Let us look at $c_{k} \alpha^{k}+c_{k+1} \alpha^{k+1} \cdots+c_{l} \alpha^{l}=0$ where $k<l ; k, l \in \mathbb{Z} ; c_{k}, c_{k+1} \ldots c_{l} \in \mathbb{C}$. We know that $\operatorname{supp}\left(\alpha^{i}\right)=\mathfrak{P}_{0} \zeta^{i}$ for $k \leqslant i \leqslant l$. Let $x \in \operatorname{supp}\left(\alpha^{s}\right)$ where $k \leqslant s \leqslant l$. Now consider $c_{k} \alpha^{k}(x)+c_{k+1} \alpha^{k+1}(x) \cdots+c_{l} \alpha^{l}(x)=0$. This implies that $c_{s} \alpha^{s}(x)=0$ as $x \in \operatorname{supp}\left(\alpha^{s}\right)$. But as $\alpha^{s}(x) \neq 0 \Longrightarrow c_{s}=0$. Hence $c_{k}=c_{k+1} \cdots=c_{l}=0$. So $\left\{\alpha^{n} \mid n \in \mathbb{Z}\right\}$ is a linearly independent set. Thus $\mathbb{C}\left[\alpha, \alpha^{-1}\right] \simeq \mathbb{C}\left[x, x^{-1}\right]$.

We have already shown before in sections 3.4 and 3.5 that $\mathcal{B}=\mathbb{C}\left[\psi, \psi^{-1}\right]$ is a sub-algebra of $\mathcal{A}=\mathcal{H}(G, \rho)$, where $\psi$ is supported on $\mathfrak{P} \zeta \mathfrak{P}$ and $\mathcal{B} \cong \mathbb{C}\left[x, x^{-1}\right]$. As $\mathcal{H}\left(L, \rho_{0}\right)=\mathbb{C}\left[\alpha, \alpha^{-1}\right] \cong \mathbb{C}\left[x, x^{-1}\right]$, so $\mathcal{B} \cong \mathcal{H}\left(L, \rho_{0}\right)$ as $\mathbb{C}$-algebras. Hence $\mathcal{H}\left(L, \rho_{0}\right)$ can be viewed as a sub-algebra of $\mathcal{H}(G, \rho)$.

Now we would like to find out how simple $\mathcal{H}\left(L, \rho_{0}\right)$-modules look like. Thus to understand them we need to find out how simple $\mathbb{C}\left[x, x^{-1}\right]$-modules look like.

\subsection{Calculation of simple $\mathcal{H}\left(L, \rho_{0}\right)$-modules}

The following Propn. is taken from Propn. 3.11 in [1].

Proposition 3.23. If $A$ is a commutative ring with identity and $S$ is a multiplicative closed subset of $A$. If $A$ is a principal ideal domain then $S^{-1} A$ is also a principal ideal domain. And also if $I$ is an ideal in $S^{-1} A$ then there exists an ideal $J$ in $A$ such that $I=J S^{-1} A$.

Lemma 3.24. $\mathbb{C}\left[x, x^{-1}\right]$ is a principal ideal domain. 
Proof. Let $A=\mathbb{C}[x]$ and $S=\left\{x^{n} \mid n \in \mathbb{N} \cup\{0\}\right\}$. Clearly, $S$ is a multiplicative closed subset of $A$ and $A$ is a principal ideal domain. Now we have $S^{-1} A=$ $\mathbb{C}\left[x, x^{-1}\right]$. From Propn. 3.23, $\mathbb{C}\left[x, x^{-1}\right]$ is a principal ideal domain.

Lemma 3.25. Any maximal ideal in $\mathbb{C}\left[x, x^{-1}\right]$ is of the form $(x-\lambda) \mathbb{C}\left[x, x^{-1}\right]$ where $\lambda \in \mathbb{C}^{\times}$.

Proof. Suppose $I$ be a proper ideal in $\mathbb{C}\left[x, x^{-1}\right]$. From Propn. 3.23, we know that $I$ is of the form $J \mathbb{C}\left[x, x^{-1}\right]$ where $J$ is an ideal in $\mathbb{C}[x]$. As $\mathbb{C}[x]$ is a principal ideal domain so $J=p(x) \mathbb{C}[x]$ for some $p(x) \in \mathbb{C}[x]$ and $\operatorname{deg} p(x)>0$. Let $\lambda \in \mathbb{C}$ be a root of $p(x)$. So $(x-\lambda) \mid p(x)$. This would imply $p(x) \mathbb{C}[x] \subseteq$ $(x-\lambda) \mathbb{C}[x]$. Hence $I=p(x) \mathbb{C}\left[x, x^{-1}\right] \subseteq(x-\lambda) \mathbb{C}\left[x, x^{-1}\right]$. But $I$ is a maximal ideal in $\mathbb{C}\left[x, x^{-1}\right]$. So $I=(x-\lambda) \mathbb{C}\left[x, x^{-1}\right]$. So any maximal ideal in $\mathbb{C}\left[x, x^{-1}\right]$ is of the form $(x-\lambda) \mathbb{C}\left[x, x^{-1}\right]$ where $\lambda \in \mathbb{C}$. But if $\lambda=0$ then $(x-\lambda)=x$ and $(x-\lambda) \mathbb{C}\left[x, x^{-1}\right]=x \mathbb{C}\left[x, x^{-1}\right]=\mathbb{C}\left[x, x^{-1}\right]$ which is not a maximal ideal. So $\lambda \in \mathbb{C}^{\times}$.

The following Propn. is taken from exercise problem 9 on page 356 in [3].

Proposition 3.26. Let $R$ be a commutative ring with identity. An $R$-module $M$ is simple $\Longleftrightarrow M \cong R / I$ for some maximal ideal $I$ in $R$.

From Propn. 3.26, every simple $\mathbb{C}\left[x, x^{-1}\right]$-module is isomorphic to $\mathbb{C}\left[x, x^{-1}\right]$ module $\frac{\mathbb{C}\left[x, x^{-1}\right]}{(x-\lambda) \mathbb{C}\left[x, x^{-1}\right]}$ for some $\lambda \in \mathbb{C}^{\times}$.

The following Propn. is taken from Propn. 3.11 in [1].

Proposition 3.27. A is a commutative ring with identity and $S$ is a multiplicative closed subset of $A$. Let $J$ be an ideal in $A$. Then we have $\frac{S^{-1} A}{J S^{-1} A} \cong \frac{A}{J}$ as $S^{-1} A$-modules. 
Let $A=\mathbb{C}[x]$ and $S=\left\{x^{n} \mid n \in \mathbb{N} \cup\{0\}\right\}$ in the Propn. 3.27. So we have $S^{-1} A=\mathbb{C}\left[x, x^{-1}\right]$. Then Propn. 3.27 says that $\frac{\mathbb{C}\left[x, x^{-1}\right]}{(x-\lambda) \mathbb{C}\left[x, x^{-1}\right]} \cong \frac{\mathbb{C}[x]}{(x-\lambda) \mathbb{C}[x]}$ as $\mathbb{C}\left[x, x^{-1}\right]$-modules, where $\lambda \in \mathbb{C}^{\times}$.

Proposition 3.28. $\frac{\mathbb{C}[x]}{(x-\lambda) \mathbb{C}[x]} \cong \mathbb{C}_{\lambda}$ as $\mathbb{C}[x]$-modules, where $\lambda \in \mathbb{C}^{\times}$and $\mathbb{C}_{\lambda}$ is the ring $\mathbb{C}$ with $\mathbb{C}[x]$-module structure given by $x . z=\lambda z$ for $z \in \mathbb{C}_{\lambda}$.

Proof. The $\mathbb{C}[x]$-module structure of $\frac{\mathbb{C}[x]}{(x-\lambda) \mathbb{C}[x]}$ is given by $p(x) \cdot \overline{q(x)}=p(\lambda) \overline{q(x)}$ where $p(x), q(x) \in \mathbb{C}[x]$. The map

$$
\phi: \frac{\mathbb{C}[x]}{(x-\lambda) \mathbb{C}[x]} \longrightarrow \mathbb{C}_{\lambda}
$$

is defined as $\phi(\overline{p(x)})=p(\lambda)$ for $p(x) \in \mathbb{C}[x]$. We shall now check that $\phi$ is a $\mathbb{C}[x]$-module homomorphism. Let $p(x), q(x) \in \mathbb{C}[x]$. Now let us consider

$$
\begin{aligned}
\phi(\overline{p(x)+q(x)}) & =\phi(\overline{(p+q)(x)}) \\
& =(p+q)(\lambda) \\
& =p(\lambda)+q(\lambda) \\
& =\phi(\overline{p(x)})+\phi(\overline{q(x)}) .
\end{aligned}
$$

Now let us look at

$$
\begin{aligned}
\phi(p(x) . q(x)) & =\phi(p(\lambda) \overline{q(x)}) \\
& =\phi(\overline{p(\lambda) q(x)}) \\
& =p(\lambda) q(\lambda) \\
& =p(\lambda) \phi(\overline{q(x)}) \\
& =p(x) \cdot \phi(\overline{q(x)}) .
\end{aligned}
$$


So $\phi$ is a homomorphism of $\mathbb{C}[x]$-modules. Let $z \in \mathbb{C}$, then there exists a polynomial $p(x) \in \mathbb{C}[x]$ such that $p(\lambda)=z$. Hence $\phi(\overline{p(x)})=p(\lambda)=z$. So $\phi$ is surjective map. Suppose if $\phi(\overline{p(x)})=\phi(\overline{q(x)})$ where $p(x), q(x) \in \mathbb{C}[x]$ then $p(\lambda)=q(\lambda)$. This implies that $(p-q)(\lambda)=0 \Longrightarrow(x-\lambda)|(p-q)(x) \Longrightarrow(x-\lambda)|$ $(p(x)-q(x)) \Longrightarrow \overline{p(x)}=\overline{q(x)}$. So $\phi$ is one-one map. Hence $\phi$ is an isomorphism of $\mathbb{C}[x]$-modules. Hence the module structure of ring $\mathbb{C}[x]$ over $\frac{\mathbb{C}[x]}{(x-\lambda)}$ is preserved for $\mathbb{C}_{\lambda}$. Therefore the $\mathbb{C}[x]$-module structure of $\mathbb{C}_{\lambda}$ is given by $x . z=\lambda z$ where $z \in \mathbb{C}_{\lambda}$.

So from Propn. 3.28, we have $\frac{\mathbb{C}[x]}{(x-\lambda)} \cong \mathbb{C}_{\lambda}$ as $\mathbb{C}[x]$-modules for $\lambda \in \mathbb{C}^{\times}$. This means that $\frac{\mathbb{C}[x]}{(x-\lambda)} \cong \mathbb{C}_{\lambda}$ as $\mathbb{C}\left[x, x^{-1}\right]$-modules for $\lambda \in \mathbb{C}^{\times}$. Recall that $\frac{\mathbb{C}\left[x, x^{-1}\right]}{(x-\lambda) \mathbb{C}\left[x, x^{-1}\right]} \cong \frac{\mathbb{C}[x]}{(x-\lambda) \mathbb{C}[x]}$ as $\mathbb{C}\left[x, x^{-1}\right]$-modules for $\lambda \in \mathbb{C}^{\times}$. Therefore $\frac{\mathbb{C}\left[x, x^{-1}\right]}{(x-\lambda) \mathbb{C}\left[x, x^{-1}\right]} \cong$ $\mathbb{C}_{\lambda}$ as $\mathbb{C}\left[x, x^{-1}\right]$-modules for $\lambda \in \mathbb{C}^{\times}$with the $\mathbb{C}\left[x, x^{-1}\right]$-module structure on $\mathbb{C}_{\lambda}$ given by $x . z=\lambda z$ where $z \in \mathbb{C}_{\lambda}$.

As $\mathcal{H}\left(L, \rho_{0}\right)=\mathbb{C}\left[\alpha, \alpha^{-1}\right]$, so the simple $\mathcal{H}\left(L, \rho_{0}\right)$-modules are same as the simple $\mathbb{C}\left[\alpha, \alpha^{-1}\right]$-modules. We have shown before that $\mathbb{C}\left[\alpha, \alpha^{-1}\right] \cong \mathbb{C}\left[x, x^{-1}\right]$ as algebras. So the distinct simple $\mathcal{H}\left(L, \rho_{0}\right)$-modules(up to isomorphism) are the various $\mathbb{C}_{\lambda}$ for $\lambda \in \mathbb{C}^{\times}$. The module structure is determined by $\alpha . z=\lambda z$ for $z \in \mathbb{C}_{\lambda}$. 


\section{Chapter 4}

\section{Final computations to answer the question}

\subsection{Calculation of $\delta_{P}(\zeta)$}

Let us recall the modulus character $\delta_{P}: P \longrightarrow \mathbb{R}_{>0}^{\times}$introduced in section 1.3. The character $\delta_{P}$ is given by $\delta_{P}(p)=\left\|\left.\operatorname{det}(A d p)\right|_{\text {Lie } U}\right\|_{F}$ for $p \in P$, where Lie $U$ is the Lie algebra of $U$. We have

$$
\begin{gathered}
U=\left\{\left[\begin{array}{ll}
1 & X \\
0 & 1
\end{array}\right] \mid X \in \mathrm{M}_{n}(E), X+{ }^{t} \bar{X}=0\right\}, \\
\text { Lie } U=\left\{\left[\begin{array}{ll}
0 & X \\
0 & 0
\end{array}\right] \mid X \in \mathrm{M}_{n}(E), X+{ }^{t} \bar{X}=0\right\} .
\end{gathered}
$$




\subsubsection{Calculation of $\delta_{P}(\zeta)$ : unramified case}

Recall $\zeta=\left[\begin{array}{cc}\varpi_{E} 1 & 0 \\ 0 & \varpi_{E}^{-1} 1\end{array}\right]$ in the unramified case. So
$(A d \zeta)\left[\begin{array}{ll}0 & X \\ 0 & 0\end{array}\right]=\zeta\left[\begin{array}{cc}0 & X \\ 0 & 0\end{array}\right] \zeta^{-1}=\left[\begin{array}{cc}0 & \varpi_{E}^{2} X \\ 0 & 0\end{array}\right]$.

Hence

$$
\begin{aligned}
\delta_{P}(\zeta) & =\left\|\left.\operatorname{det}(\operatorname{Ad} \zeta)\right|_{\operatorname{Lie} U}\right\|_{F} \\
& =\left\|\varpi_{E}^{2\left(\operatorname{dim} m_{F}(\operatorname{Lie} U)\right)}\right\|_{F} \\
& =\left\|\varpi_{E}^{2 n^{2}}\right\|_{F} \\
& =\left\|\varpi_{F}^{22^{2}}\right\|_{F} \\
& =q^{-2 n^{2}} .
\end{aligned}
$$

\subsubsection{Calculation of $\delta_{P}(\zeta)$ : ramified case}

Recall $\zeta=\left[\begin{array}{cc}\varpi_{E} 1 & 0 \\ 0 & -\varpi_{E}^{-1} 1\end{array}\right]$ in the ramified case. So

$$
(A d \zeta)\left[\begin{array}{cc}
0 & X \\
0 & 0
\end{array}\right]=\zeta\left[\begin{array}{cc}
0 & X \\
0 & 0
\end{array}\right] \zeta^{-1}=\left[\begin{array}{cc}
0 & -\varpi_{E}^{2} X \\
0 & 0
\end{array}\right] .
$$

Hence

$$
\begin{aligned}
\delta_{P}(\zeta) & =\left\|\left.\operatorname{det}(\operatorname{Ad} \zeta)\right|_{\operatorname{Lie} U}\right\|_{F} \\
& =\left\|-\varpi_{E}^{2\left(\operatorname{dim} m_{F}(\operatorname{Lie} U)\right)}\right\|_{F} \\
& =\left\|\varpi_{E}^{22^{2}}\right\|_{F} \\
& =\left\|\varpi_{F}^{n^{2}}\right\|_{F}
\end{aligned}
$$




$$
=q^{-n^{2}}
$$

\subsection{Understanding the map $T_{P}$}

Let us denote the set of $(\mathfrak{P}, P)$-positive elements by $\mathcal{I}^{+}$. Thus

$$
\mathcal{I}^{+}=\left\{x \in L \mid x \mathfrak{P}_{+} x^{-1} \subseteq \mathfrak{P}_{+}, x^{-1} \mathfrak{P}_{-} x \subseteq \mathfrak{P}_{-}\right\}
$$

where $\mathfrak{P}_{+}=\mathfrak{P} \cap U, \mathfrak{P}_{-}=\mathfrak{P} \cap \bar{U}$. We have

$$
\mathcal{H}^{+}\left(L, \rho_{0}\right)=\left\{f \in \mathcal{H}\left(L, \rho_{0}\right) \mid \operatorname{supp} f \subseteq \mathfrak{P}_{0} \mathcal{I}^{+} \mathfrak{P}_{0}\right\}
$$

Note $\zeta \in \mathcal{I}^{+}$, so $\mathcal{H}^{+}\left(L, \rho_{0}\right)=\mathbb{C}[\alpha]$. The following discussion is taken from pages 612-619 in [2]. Let $W$ be space of $\rho_{0}$. Let $f \in \mathcal{H}^{+}\left(L, \rho_{0}\right)$ with support of $f$ being $\mathfrak{P}_{0} x \mathfrak{P}_{0}$ for $x \in \mathcal{I}^{+}$. The map $F \in \mathcal{H}(G, \rho)$ is supported on $\mathfrak{P} x \mathfrak{P}$ and $f(x)=F(x)$. The algebra embedding

$$
T^{+}: \mathcal{H}^{+}\left(L, \rho_{0}\right) \longrightarrow \mathcal{H}(G, \rho)
$$

is given by $T^{+}(f)=F$.

Recall support of $\alpha \in \mathcal{H}^{+}\left(L, \rho_{0}\right)$ is $\mathfrak{P}_{0} \zeta$. Let $T^{+}(\alpha)=\psi$, where $\psi \in \mathcal{H}(G, \rho)$ has support $\mathfrak{P} \zeta \mathfrak{P}$ and $\alpha(\zeta)=\psi(\zeta)=1_{W^{\vee}}$. As $T^{+}(\alpha)=\psi$ is invertible, so from Propn. 1.7 we can conclude that $T^{+}$extends to an embedding of algebras

$$
t: \mathcal{H}\left(L, \rho_{0}\right) \longrightarrow \mathcal{H}(G, \rho) \text {. }
$$

Let $\phi \in \mathcal{H}\left(L, \rho_{0}\right)$ and $m \in \mathbb{N}$ is chosen such that $\alpha^{m} \phi \in \mathcal{H}^{+}\left(L, \rho_{0}\right)$. The map 
$t$ is then given by $t(\phi)=\psi^{-m} T^{+}\left(\alpha^{m} \phi\right)$. For $\phi \in \mathcal{H}\left(L, \rho_{0}\right)$, the map

$$
t_{P}: \mathcal{H}\left(L, \rho_{0}\right) \longrightarrow \mathcal{H}(G, \rho)
$$

is given by $t_{P}(\phi)=t\left(\phi \delta_{P}\right)$, where $\phi \delta_{P} \in \mathcal{H}\left(L, \rho_{0}\right)$ and is the map

$$
\phi \delta_{P}: L \longrightarrow \operatorname{End}_{\mathbb{C}}\left(\rho_{0}^{\vee}\right)
$$

given by $\left(\phi \delta_{P}\right)(l)=\phi(l) \delta_{P}(l)$ for $l \in L$. As $\alpha \in \mathcal{H}\left(L, \rho_{0}\right)$ we have

$$
\begin{aligned}
t_{P}(\alpha)(\zeta) & =t\left(\alpha \delta_{P}\right)(\zeta) \\
& =T^{+}\left(\alpha \delta_{P}\right)(\zeta) \\
& =\delta_{P}(\zeta) T^{+}(\alpha)(\zeta) \\
& =\delta_{P}(\zeta) \psi(\zeta) \\
& =\delta_{P}(\zeta) 1_{W^{\vee}}
\end{aligned}
$$

Let $\mathcal{H}\left(L, \rho_{0}\right)$-Mod denote the category of $\mathcal{H}\left(L, \rho_{0}\right)$-modules and $\mathcal{H}(G, \rho)$-Mod denote the category of $\mathcal{H}(G, \rho)$-modules. The map $t_{P}$ induces a functor $\left(t_{P}\right)_{*}$ given by

$$
\left(t_{P}\right)_{*}: \mathcal{H}\left(L, \rho_{0}\right)-\operatorname{Mod} \longrightarrow \mathcal{H}(G, \rho)-\operatorname{Mod}
$$

For $M$ an $\mathcal{H}\left(L, \rho_{0}\right)$-module,

$$
\left(t_{P}\right)_{*}(M)=\operatorname{Hom}_{\mathcal{H}\left(L, \rho_{0}\right)}(\mathcal{H}(G, \rho), M)
$$

where $\mathcal{H}(G, \rho)$ is viewed as a $\mathcal{H}\left(L, \rho_{0}\right)$-module via $t_{P}$. The action of $\mathcal{H}(G, \rho)$ on 
$\left(t_{P}\right)_{*}(M)$ is given by

$$
h^{\prime} \psi\left(h_{1}\right)=\psi\left(h_{1} h^{\prime}\right)
$$

where $\psi \in\left(t_{P}\right)_{*}(M), h_{1}, h^{\prime} \in \mathcal{H}(G, \rho)$.

Let $\tau \in \mathfrak{R}^{[L, \pi]_{L}}(L)$ then functor $m_{L}: \mathfrak{R}^{[L, \pi]_{L}}(L) \longrightarrow \mathcal{H}\left(L, \rho_{0}\right)-M o d$ is given by $m_{L}(\tau)=\operatorname{Hom}_{\mathfrak{P}_{0}}\left(\rho_{0}, \tau\right)$. The functor $m_{L}$ is an equivalence of categories. Let $f \in m_{L}(\tau), \gamma \in \mathcal{H}\left(L, \rho_{0}\right)$ and $w \in W$. The action of $\mathcal{H}\left(L, \rho_{0}\right)$ on $m_{L}(\tau)$ is given by $(\gamma \cdot f)(w)=\int_{L} \tau(l) f\left(\gamma^{\vee}\left(l^{-1}\right) w\right) d l$. Here $\gamma^{\vee}$ is defined on $L$ by $\gamma^{\vee}\left(l^{-1}\right)=\gamma(l)^{\vee}$ for $l \in L$. Let $\tau^{\prime} \in \mathfrak{R}^{[L, \pi]_{G}}(G)$ then the functor $m_{G}: \mathfrak{R}^{[L, \pi]_{G}}(G) \longrightarrow \mathcal{H}(G, \rho)-\operatorname{Mod}$ is given by $m_{G}\left(\tau^{\prime}\right)=\operatorname{Hom}_{\mathfrak{P}}\left(\rho, \tau^{\prime}\right)$. The functor $m_{G}$ is an equivalence of categories. From Corollary 8.4 in [2], the functors $m_{L}, m_{G}, \operatorname{Ind}_{P}^{G},\left(t_{P}\right)_{*}$ fit into the following commutative diagram:

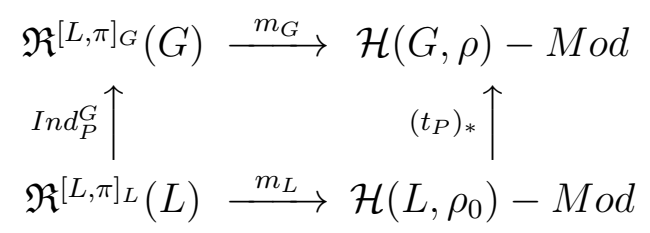

If $\tau \in \mathfrak{R}^{[L, \pi]_{L}}(L)$ then from the above commutative diagram, we see that $\left(t_{P}\right)_{*}\left(m_{L}(\tau)\right) \cong m_{G}\left(\operatorname{Ind} d_{P}^{G} \tau\right)$ as $\mathcal{H}(G, \rho)$-modules. Replacing $\tau$ by $\left(\tau \otimes \delta_{P}^{1 / 2}\right)$ in the above expression, $\left(t_{P}\right)_{*}\left(m_{L}\left(\tau \otimes \delta_{P}^{1 / 2}\right)\right) \cong m_{G}\left(\operatorname{Ind}_{P}^{G}\left(\tau \otimes \delta_{P}^{1 / 2}\right)\right)$ as $\mathcal{H}(G, \rho)$ modules. As $\operatorname{Ind}_{P}^{G}\left(\tau \otimes \delta_{P}^{1 / 2}\right)=\iota_{P}^{G}(\tau)$, we have $\left(t_{P}\right)_{*}\left(m_{L}\left(\tau \otimes \delta_{P}^{1 / 2}\right)\right) \cong m_{G}\left(\iota_{P}^{G}(\tau)\right)$ as $\mathcal{H}(G, \rho)$-modules.

Our aim is to find an algebra embedding $T_{P}: \mathcal{H}\left(L, \rho_{0}\right) \longrightarrow \mathcal{H}(G, \rho)$ such that the following diagram commutes: 


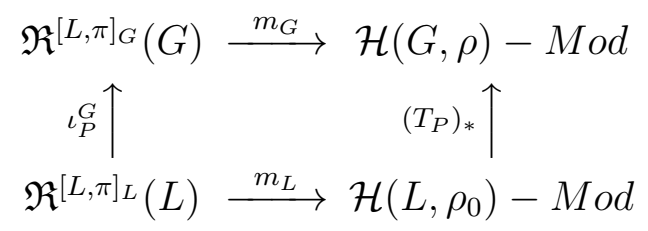

Let $\tau \in \mathfrak{R}^{[L, \pi]_{L}}(L)$ then $m_{L}(\tau) \in \mathcal{H}\left(L, \rho_{0}\right)$ - Mod. The functor $\left(T_{P}\right)_{*}$ is defined as below:

$$
\left(T_{P}\right)_{*}\left(m_{L}(\tau)\right)=\left\{\begin{array}{l|l}
\psi: \mathcal{H}(G, \rho) \rightarrow m_{L}(\tau) & \begin{array}{l}
h \psi\left(h_{1}\right)=\psi\left(T_{P}(h) h_{1}\right) \text { where } \\
h \in \mathcal{H}\left(L, \rho_{0}\right), h_{1} \in \mathcal{H}(G, \rho)
\end{array}
\end{array}\right\}
$$

From the above commutative diagram, we see that $\left(T_{P}\right)_{*}\left(m_{L}(\tau)\right) \cong m_{G}\left(\iota_{P}^{G}(\tau)\right)$ as $\mathcal{H}(G, \rho)$-modules. Recall that $\left(t_{P}\right)_{*}\left(m_{L}\left(\tau \otimes \delta_{P}^{1 / 2}\right)\right) \cong m_{G}\left(\iota_{P}^{G}(\tau)\right)$ as $\mathcal{H}(G, \rho)$ modules. Hence we have to find an algebra embedding $T_{p}: \mathcal{H}\left(L, \rho_{0}\right) \longrightarrow \mathcal{H}(G, \rho)$ such that $\left(T_{P}\right)_{*}\left(m_{L}(\tau)\right) \cong\left(t_{P}\right)_{*}\left(m_{L}\left(\tau \otimes \delta_{P}^{1 / 2}\right)\right)$ as $\mathcal{H}(G, \rho)$-modules.

Proposition 4.1. The map $T_{P}$ is given by $T_{P}(\phi)=t_{P}\left(\phi \delta_{P}^{-1 / 2}\right)$ for $\phi \in \mathcal{H}\left(L, \rho_{0}\right)$ so that we have $\left(T_{P}\right)_{*}\left(m_{L}(\tau)\right)=\left(t_{P}\right)_{*}\left(m_{L}\left(\tau \otimes \delta_{P}^{1 / 2}\right)\right)$ as $\mathcal{H}(G, \rho)$ - modules.

Proof. Let $W$ be space of $\rho_{0}$. The vector spaces for $m_{L}\left(\tau \delta_{P}^{1 / 2}\right)$ and $m_{L}(\tau)$ are the same. Let $f \in m_{L}(\tau)=\operatorname{Hom}_{\mathfrak{P}_{0}}\left(\rho_{0}, \tau\right), \gamma \in \mathcal{H}\left(L, \rho_{0}\right)$ and $w \in W$. Recall the action of $\mathcal{H}\left(L, \rho_{0}\right)$ on $m_{L}(\tau)$ is given by

$$
(\gamma \cdot f)(w)=\int_{L} \tau(l) f\left(\gamma^{\vee}\left(l^{-1}\right) w\right) d l
$$

Let $f^{\prime} \in m_{L}\left(\tau \delta_{P}^{1 / 2}\right)=\operatorname{Hom}_{\mathfrak{P}_{0}}\left(\rho_{0}, \tau \delta_{P}^{1 / 2}\right), \gamma \in \mathcal{H}\left(L, \rho_{0}\right)$ and $w \in W$. Recall the action of $\mathcal{H}\left(L, \rho_{0}\right)$ on $m_{L}\left(\tau \delta_{P}^{1 / 2}\right)$ is given by

$$
\left(\gamma \cdot f^{\prime}\right)(w)=\int_{L}\left(\tau \delta_{P}^{1 / 2}\right)(l) f^{\prime}\left(\gamma^{\vee}\left(l^{-1}\right) w\right) d l=\int_{L} \tau(l) \delta_{P}^{1 / 2}(l) f^{\prime}\left(\gamma^{\vee}\left(l^{-1}\right) w\right) d l
$$


Now $f^{\prime}$ is a linear transformation from space of $\rho_{0}$ to space of $\tau \delta_{P}^{1 / 2}$. As $\delta_{P}^{1 / 2}(l) \in$ $\mathbb{C}^{\times}$, so $\delta_{P}^{1 / 2}(l) f^{\prime}\left(\gamma^{\vee}\left(l^{-1}\right) w\right)=f^{\prime}\left(\delta_{P}^{1 / 2}(l) \gamma^{\vee}\left(l^{-1}\right) w\right)$. Hence we have

$$
\left(\gamma \cdot f^{\prime}\right)(w)=\int_{L} \tau(l) f^{\prime}\left(\delta_{P}^{1 / 2}(l) \gamma^{\vee}\left(l^{-1}\right) w\right) d l=\int_{L} \tau(l) f^{\prime}\left(\delta_{P}^{1 / 2}(l) \gamma(l)^{\vee} w\right) d l .
$$

Further as $\delta_{P}^{1 / 2}(l) \in \mathbb{C}^{\times}$, so $\delta_{P}^{1 / 2}(l)(\gamma(l))^{\vee}=\left(\delta_{P}^{1 / 2} \gamma\right)(l)^{\vee}$. Therefore

$$
\left(\gamma \cdot f^{\prime}\right)(w)=\int_{L} \tau(l) f^{\prime}\left(\left(\delta_{P}^{1 / 2} \gamma\right)(l)^{\vee} w\right) d l=\left(\delta_{P}^{1 / 2} \gamma\right) \cdot f^{\prime}(w)
$$

Hence we can conclude that the action of $\gamma \in \mathcal{H}\left(L, \rho_{0}\right)$ on $f^{\prime} \in m_{L}\left(\tau \delta_{P}^{1 / 2}\right)$ is same as the action of $\delta_{P}^{1 / 2} \gamma \in \mathcal{H}\left(L, \rho_{0}\right)$ on $f^{\prime} \in m_{L}(\tau)$. So we have $\left(T_{P}\right)_{*}\left(m_{L}(\tau)\right)=$ $\left(t_{P}\right)_{*}\left(m_{L}\left(\tau \otimes \delta_{P}^{1 / 2}\right)\right)$ as $\mathcal{H}(G, \rho)$ - modules.

From Propn. 4.1, $T_{P}(\alpha)=t_{P}\left(\alpha \delta_{P}^{-1 / 2}\right)$. So we have

$$
\begin{aligned}
T_{P}(\alpha) & =t_{P}\left(\alpha \delta_{P}^{-1 / 2}\right) \\
& =t\left(\alpha \delta_{P}^{-1 / 2} \delta_{P}\right) \\
& =t\left(\alpha \delta_{P}^{1 / 2}\right) \\
& =T^{+}\left(\alpha \delta_{P}^{1 / 2}\right)
\end{aligned}
$$

Hence

$$
\begin{aligned}
T_{P}(\alpha)(\zeta) & =T^{+}\left(\alpha \delta_{P}^{1 / 2}\right)(\zeta) \\
& =\delta_{P}^{1 / 2}(\zeta) T^{+}(\alpha)(\zeta) \\
& =\delta_{P}^{1 / 2}(\zeta) \alpha(\zeta)
\end{aligned}
$$




$$
=\delta_{P}^{1 / 2}(\zeta) 1_{W}
$$

Thus $T_{P}(\alpha)(\zeta)=\delta_{P}^{1 / 2}(\zeta) 1_{W^{\vee}}$ with $\operatorname{supp}\left(T_{P}(\alpha)\right)=\operatorname{supp}\left(t_{P}(\alpha)\right)=\mathfrak{P} \zeta \mathfrak{P}$.

\subsubsection{Calculation of $\left(\phi_{0} * \phi_{1}\right)(\zeta)$}

In this section we calculate $\left(\phi_{0} * \phi_{1}\right)(\zeta)$. Let $g_{i}=q^{-n / 2} \phi_{i}$ for $i=0,1$ in the unramified case and $g_{i}=q^{-n / 4} \phi_{i}$ for $i=0,1$ in the ramified case. Determining $\left(\phi_{0} * \phi_{1}\right)(\zeta)$ would be useful in showing $g_{0} * g_{1}=T_{P}(\alpha)$ in both ramified and unramified cases. From now on, we assume without loss of generality that $\operatorname{volP}_{0}=\operatorname{volP}_{-}=\operatorname{volP}_{+}=1$. Thus we have volP $=1$.

Lemma 4.2. $\operatorname{supp}\left(\phi_{0} * \phi_{1}\right)=\mathfrak{P} \zeta \mathfrak{P}=\mathfrak{P} w_{0} w_{1} \mathfrak{P}$.

Proof. We first claim that $\operatorname{supp}\left(\phi_{0} * \phi_{1}\right) \subseteq \mathfrak{P} w_{0} \mathfrak{P} w_{1} \mathfrak{P}$. Suppose $z \in \operatorname{supp}\left(\phi_{0} * \phi_{1}\right)$ then $\left(\phi_{0} * \phi_{1}\right)(z)=\int_{G} \phi_{0}\left(z r^{-1}\right) \phi_{1}(r) d r \neq 0$. This would imply that there exists an $r \in G$ such that $\phi_{0}\left(z r^{-1}\right) \phi_{1}(r) \neq 0$. As $\phi_{0}\left(z r^{-1}\right) \phi_{1}(r) \neq 0$, this means that $\phi_{0}\left(z r^{-1}\right) \neq 0, \phi_{1}(r) \neq 0$. But $\phi_{0}\left(z r^{-1}\right) \neq 0$ would imply that $z r^{-1} \in \mathfrak{P} w_{0} \mathfrak{P}$ and $\phi_{1}(r) \neq 0$ would imply that $r \in \mathfrak{P} w_{1} \mathfrak{P}$. So $z=\left(z r^{-1}\right)(r) \in\left(\mathfrak{P} w_{0} \mathfrak{P}\right)\left(\mathfrak{P} w_{1} \mathfrak{P}\right)=$ $\left(\operatorname{supp} \phi_{0}\right)\left(\operatorname{supp} \phi_{1}\right)=\mathfrak{P} w_{0} \mathfrak{P} w_{1} \mathfrak{P}$. Hence $\operatorname{supp}\left(\phi_{0} * \phi_{1}\right) \subseteq \mathfrak{P} w_{0} \mathfrak{P} w_{1} \mathfrak{P}$. Let us recall $\mathfrak{P}_{0}, \mathfrak{P}_{+}, \mathfrak{P}_{-}$.

$$
\begin{gathered}
\mathfrak{P}_{0}=\left\{\left[\begin{array}{cc}
a & 0 \\
0 & { }^{t} \bar{a}^{-1}
\end{array}\right] \mid a \in \mathrm{GL}_{n}\left(\mathfrak{O}_{E}\right)\right\}, \\
\mathfrak{P}_{+}=\left\{\left[\begin{array}{ll}
1 & X \\
0 & 1
\end{array}\right] \mid X \in \mathrm{M}_{n}\left(\mathfrak{O}_{E}\right), X+{ }^{t} \bar{X}=0\right\}, \\
\mathfrak{P}_{-}=\left\{\left[\begin{array}{ll}
1 & 0 \\
X & 1
\end{array}\right] \mid X \in \varpi_{E} \mathrm{M}_{n}\left(\mathfrak{O}_{E}\right), X+{ }^{t} \bar{X}=0\right\} .
\end{gathered}
$$


It is easy observe that $w_{0} \mathfrak{P}_{-} w_{0}^{-1} \subseteq \mathfrak{P}_{+}, w_{0} \mathfrak{P}_{0} w_{0}^{-1}=\mathfrak{P}_{0}, w_{1}^{-1} \mathfrak{P}_{+} w_{1} \subseteq \mathfrak{P}_{-}$.

Now we have

$$
\begin{aligned}
\mathfrak{P} w_{0} \mathfrak{P} w_{1} \mathfrak{P} & =\mathfrak{P} w_{0} \mathfrak{P}_{-} \mathfrak{P}_{0} \mathfrak{P}_{+} w_{1} \mathfrak{P} \\
& =\mathfrak{P} w_{0} \mathfrak{P}_{-} w_{0}^{-1} w_{0} \mathfrak{P}_{0} w_{0}^{-1} w_{0} w_{1} w_{1}^{-1} \mathfrak{P}_{+} w_{1} \mathfrak{P} \\
& \subseteq \mathfrak{P P} \mathfrak{P}_{0} w_{0} w_{1} \mathfrak{P}-\mathfrak{P} \\
& =\mathfrak{P} w_{0} w_{1} \mathfrak{P} \\
& =\mathfrak{P} \zeta \mathfrak{P} .
\end{aligned}
$$

So $\mathfrak{P} w_{0} \mathfrak{P} w_{1} \mathfrak{P} \subseteq \mathfrak{P} w_{0} w_{1} \mathfrak{P}=\mathfrak{P} \zeta \mathfrak{P}$. On the contrary, as $1 \in \mathfrak{P}$, so $\mathfrak{P} \zeta \mathfrak{P}=$ $\mathfrak{P} w_{0} w_{1} \mathfrak{P} \subseteq \mathfrak{P} w_{0} \mathfrak{P} w_{1} \mathfrak{P}$. Hence we have $\mathfrak{P} w_{0} \mathfrak{P} w_{1} \mathfrak{P}=\mathfrak{P} w_{0} w_{1} \mathfrak{P}=\mathfrak{P} \zeta \mathfrak{P}$. Therefore $\operatorname{supp}\left(\phi_{0} * \phi_{1}\right) \subseteq \mathfrak{P} w_{0} \mathfrak{P} w_{1} \mathfrak{P}=\mathfrak{P} w_{0} w_{1} \mathfrak{P}=\mathfrak{P} \zeta \mathfrak{P}$. This implies $\operatorname{supp}\left(\phi_{0} * \phi_{1}\right)=$ $\varnothing$ or $\mathfrak{P} \zeta \mathfrak{P}$. But if $\operatorname{supp}\left(\phi_{0} * \phi_{1}\right)=\varnothing$ then $\left(\phi_{0} * \phi_{1}\right)=0$ which is a contradiction. Thus $\operatorname{supp}\left(\phi_{0} * \phi_{1}\right)=\mathfrak{P} \zeta \mathfrak{P}$.

For $r \in \mathbb{Z}$ let

$$
\begin{aligned}
& K_{-, r}=\left\{\left[\begin{array}{ll}
1 & 0 \\
X & 1
\end{array}\right] \mid X \in \mathrm{M}_{n}\left(\mathbf{p}_{E}^{r}\right), X+{ }^{t} \bar{X}=0\right\}, \\
& K_{+, r}=\left\{\left[\begin{array}{ll}
1 & X \\
0 & 1
\end{array}\right] \mid X \in \mathrm{M}_{n}\left(\mathbf{p}_{E}^{r}\right), X+{ }^{t} \bar{X}=0\right\} .
\end{aligned}
$$

Proposition 4.3. $\left(\phi_{0} * \phi_{1}\right)(\zeta)=\phi_{0}\left(w_{0}\right) \phi_{1}\left(w_{1}\right)$.

Proof. From Lemma 4.2, $\operatorname{supp}\left(\phi_{0} * \phi_{1}\right)=\mathfrak{P} \zeta \mathfrak{P}=\mathfrak{P} w_{0} w_{1} \mathfrak{P}$. So now let us consider

$$
\left(\phi_{0} * \phi_{1}\right)(\zeta)=\left(\phi_{0} * \phi_{1}\right)\left(w_{0} w_{1}\right)
$$




$$
\begin{aligned}
& =\int_{G} \phi_{0}(y) \phi_{1}\left(y^{-1} \zeta\right) d y \\
& =\int_{\mathfrak{P} w_{0} \mathfrak{P}} \phi_{0}(y) \phi_{1}\left(y^{-1} \zeta\right) d y .
\end{aligned}
$$

We know that $\mathfrak{P} w_{0} \mathfrak{P}=\underset{z \in \mathfrak{P} w_{0} \mathfrak{P} / \mathfrak{P}}{\amalg} z \mathfrak{P}$. Let $y=z p \in z \mathfrak{P}$. So we have

$$
\begin{aligned}
\phi_{0}(y) \phi_{1}\left(y^{-1} \zeta\right) & =\phi_{0}(z p) \phi_{1}\left(p^{-1} z^{-1} \zeta\right) \\
& =\phi_{0}(z) \rho^{\vee}(p) \rho^{\vee}\left(p^{-1}\right) \phi_{1}\left(z^{-1} \zeta\right) \\
& =\phi_{0}(z) \phi_{1}\left(z^{-1} \zeta\right) .
\end{aligned}
$$

Hence

$$
\left(\phi_{0} * \phi_{1}\right)(\zeta)=\sum_{z \in \mathfrak{P} w_{0} \mathfrak{P} / \mathfrak{P}} \phi_{0}(z) \phi_{1}\left(z^{-1} \zeta\right) \operatorname{Vol} \mathfrak{P}=\sum_{z \in \mathfrak{P} w_{0} \mathfrak{P} / \mathfrak{P}} \phi_{0}(z) \phi_{1}\left(z^{-1} \zeta\right)
$$

Let $\alpha: \mathfrak{P} / w_{0} \mathfrak{P} w_{0}^{-1} \cap \mathfrak{P} \longrightarrow \mathfrak{P} w_{0} \mathfrak{P} / \mathfrak{P}$ be the map given by $\alpha\left(x\left(w_{0} \mathfrak{P} w_{0}^{-1} \cap\right.\right.$ $\mathfrak{P}))=x w_{0} \mathfrak{P}$ where $x \in \mathfrak{P}$. We can observe that the map $\alpha$ is bijective. So $\mathfrak{P} / w_{0} \mathfrak{P} w_{0}^{-1} \cap \mathfrak{P}$ is in bijection with $\mathfrak{P} w_{0} \mathfrak{P} / \mathfrak{P}$.

Hence

$$
\left(\phi_{0} * \phi_{1}\right)(\zeta)=\sum_{x \in \mathfrak{P} / w_{0} \mathfrak{\Re} w_{0}^{-1} \cap \mathfrak{P}} \phi_{0}\left(x w_{0}\right) \phi_{1}\left(w_{0}^{-1} x^{-1} \zeta\right) .
$$

From Iwahori factorization of $\mathfrak{P}$ we have $\mathfrak{P}=\mathfrak{P}_{-} \mathfrak{P}_{0} \mathfrak{P}_{+}=K_{-, 1} \mathfrak{P}_{0} K_{+, 0}$. Therefore $w_{0} \mathfrak{P} w_{0}^{-1}={ }^{w_{0}} \mathfrak{P}={ }^{w_{0}} K_{-, 1}^{w_{0}} \mathfrak{P}_{0}^{w_{0}} K_{+, 0}=K_{+, 1} \mathfrak{P}_{0} K_{-, 0}$. So $\mathfrak{P}_{0} \cap w_{0} \mathfrak{P} w_{0}^{-1}=$ $\mathfrak{P} \cap w_{0}^{w_{0}} \mathfrak{P}=K_{+, 1} \mathfrak{P}_{0} K_{-, 1}$. Let $\beta: \mathfrak{P} / w_{0} \mathfrak{P} w_{0}^{-1} \cap \mathfrak{P} \longrightarrow K_{+, 0} / K_{+, 1}$ be the map given by $\beta\left(x\left(\mathfrak{P} \cap{ }^{w_{0}} \mathfrak{P}\right)\right)=x_{+} K_{+, 1}$ where $x \in \mathfrak{P}$ and $x=x_{+} p x_{-}, x_{+} \in \mathfrak{P}_{+}, p \in$ $\mathfrak{P}_{0}, x_{-} \in \mathfrak{P}_{-}$. We can observe that the map $\beta$ is bijective. So $\mathfrak{P} / w_{0} \mathfrak{P} w_{0}^{-1} \cap \mathfrak{P}$ is in bijection with $K_{+, 0} / K_{+, 1}$. 
Therefore

$$
\begin{aligned}
\left(\phi_{0} * \phi_{1}\right)(\zeta) & =\sum_{x_{+} \in K_{+, 0} / K_{+, 1}} \phi_{0}\left(x_{+} w_{0}\right) \phi_{1}\left(w_{0}^{-1} x_{+}^{-1} \zeta\right) \\
& =\sum_{x_{+} \in K_{+, 0} / K_{+, 1}} \rho^{\vee}\left(x_{+}\right) \phi_{0}\left(w_{0}\right) \phi_{1}\left(w_{0}^{-1} x_{+}^{-1} \zeta\right)
\end{aligned}
$$

As $\rho^{\vee}$ is trivial on $\mathfrak{P}_{+}$and $x_{+} \in \mathfrak{P}_{+}$so we have

$$
\left(\phi_{0} * \phi_{1}\right)(\zeta)=\sum_{x_{+} \in K_{+, 0} / K_{+, 1}} \phi_{0}\left(w_{0}\right) \phi_{1}\left(w_{0}^{-1} x_{+}^{-1} \zeta\right) .
$$

The terms in above summation which do not vanish are the ones for which $w_{0}^{-1} x_{+}^{-1} \zeta \in \mathfrak{P} w_{1} \mathfrak{P} \Longrightarrow x_{+}^{-1} \in w_{0} \mathfrak{P} w_{1} \mathfrak{P} \zeta^{-1} \Longrightarrow x_{+} \in \zeta \mathfrak{P} w_{1}^{-1} \mathfrak{P} w_{0}^{-1} \Longrightarrow w_{0}^{-1} x_{+} w_{0} \in$ $w_{1} \mathfrak{P} w_{1}^{-1} \mathfrak{P} . \quad$ It is clear $w_{1} \mathfrak{P} w_{1}^{-1} \mathfrak{P}=\left({ }^{w_{1}} \mathfrak{P}\right)(\mathfrak{P}) . \quad$ As $^{w_{1}} \mathfrak{P}={ }^{w_{1}} K_{-, 1}^{w_{1}} \mathfrak{P}_{0}^{w_{1}} K_{+, 0}=$ $K_{-, 2} \mathfrak{P}_{0} K_{+,-1}$, so $w_{1} \mathfrak{P} w_{1}^{-1} \mathfrak{P}=\left({ }^{w_{1}} \mathfrak{P}\right)(\mathfrak{P})=K_{-, 2} \mathfrak{P}_{0} K_{+,-1} \mathfrak{P}_{0} K_{-, 1}$. Hence we have $w_{0}^{-1} x_{+} w_{0} \in K_{-, 2} \mathfrak{P}_{0} K_{+,-1} \mathfrak{P}_{0} K_{-, 1} \Longrightarrow w_{0}^{-1} x_{+} w_{0}=k_{-} p_{0} k_{+} k_{-}^{\prime}$ where $k_{-} \in$ $K_{-, 2}, k_{+} \in K_{+,-1}, k_{-}^{\prime} \in K_{-, 1}, p_{0} \in \mathfrak{P}_{0}$. Hence we have $p_{0} k_{+}=k_{-}^{-1} w_{0}^{-1} x_{+} w_{0} k_{-}^{\prime-1}$. Now as $w_{0}^{-1} x_{+} w_{0} \in K_{-, 0}, k_{-}^{-1} \in K_{-, 2}, k_{-}^{\prime-1} \in K_{-, 1}$, so $k_{-}^{-1} w_{0}^{-1} x_{+} w_{0} k_{-}^{\prime-1} \in K_{-, 0}$ and $p_{0} k_{+} \in \mathfrak{P}_{0} K_{+,-1}$. But we know that $K_{-, 0} \cap \mathfrak{P}_{0} K_{+,-1}=1 \Longrightarrow p_{0} k_{+}=1 \Longrightarrow$ $w_{0}^{-1} x_{+} w_{0}=k_{-} k_{-}^{\prime} \in K_{-, 1} \Longrightarrow x_{+} \in w_{0} K_{-, 1} w_{0}^{-1}=K_{+, 1}$. As $x_{+} \in K_{+, 1}$, so only the trivial coset contributes to the above summation. Hence

$$
\left(\phi_{0} * \phi_{1}\right)(\zeta)=\phi_{0}\left(w_{0}\right) \phi_{1}\left(w_{0}^{-1} \zeta\right)=\phi_{0}\left(w_{0}\right) \phi_{1}\left(w_{1}\right)
$$

\subsubsection{Relation between $g_{0}, g_{1}$ and $T_{P}(\alpha)$ : unramified case}

Recall that $\mathcal{H}(G, \rho)=\left\langle\phi_{0}, \phi_{1}\right\rangle$ where $\phi_{0}$ is supported on $\mathfrak{P} w_{0} \mathfrak{P}$ and $\phi_{1}$ is supported on $\mathfrak{P} w_{1} \mathfrak{P}$ respectively with $\phi_{i}^{2}=q^{n}+\left(q^{n}-1\right) \phi_{i}$ for $i=0,1$. In this 
section we show that $g_{0} * g_{1}=T_{P}(\alpha)$, where $g_{i}=q^{-n / 2} \phi_{i}$ for $i=0,1$.

Proposition 4.4. $g_{0} g_{1}=T_{P}(\alpha)$.

Proof. Let us choose $\psi_{i} \in \mathcal{H}(G, \rho)$ for $i=0,1$ such that $\operatorname{supp}\left(\psi_{i}\right)=\mathfrak{P} w_{i} \mathfrak{P}$ for $i=$ 0,1 . So $\phi_{i}$ is a scalar multiple of $\psi_{i}$ for $i=0,1$. Hence $\phi_{i}=\lambda_{i} \psi_{i}$ where $\lambda_{i} \in \mathbb{C}^{\times}$for $i=0,1$. Let $\psi_{i}\left(w_{i}\right)=A \in \operatorname{Hom}_{\mathfrak{P} \cap{ }^{w_{i}} \mathfrak{P}}\left({ }^{w_{i}} \rho^{\vee}, \rho^{\vee}\right)$ for $i=0,1$ and $W$ be the space of $\rho$. So $A^{2}=1_{W^{\vee}}$. From Propn. 4.3 , we have $\left(\psi_{0} * \psi_{1}\right)(\zeta)=\psi_{0}\left(w_{0}\right) \psi_{1}\left(w_{1}\right)=A^{2}=$ $1_{W^{\vee}}$. Now let $\psi_{i}$ satisfies the quadratic relation given by $\psi_{i}^{2}=a \psi_{i}+b$ where $a, b \in \mathbb{R}$ for $i=0,1$. As $\psi_{i}^{2}=a \psi_{i}+b \Longrightarrow\left(-\psi_{i}\right)^{2}=(-a)\left(-\psi_{i}\right)+b$, so $a$ can be arranged such that $a>0$. We can see that $1 \in \mathcal{H}(G, \rho)$ is defined as below:

$$
1(x)= \begin{cases}0, & \text { if } x \notin \mathfrak{P} ; \\ \rho^{\vee}(x) & \text { if } x \in \mathfrak{P} .\end{cases}
$$

Let us consider $\psi_{i}^{2}(1)=\int_{G} \psi_{i}(y) \psi_{i}\left(y^{-1}\right) d y$ for $i=0,1$. Now let $y=p w_{i} p^{\prime}$ where $p, p^{\prime} \in \mathfrak{P}$ for $i=0,1$. So we have

$$
\begin{aligned}
\psi_{i}^{2}(1) & =\int_{\mathfrak{P} w_{i} \mathfrak{P}} \psi_{i}\left(p w_{i} p^{\prime}\right) \psi_{i}\left(p^{\prime}-1 w_{i}^{-1} p^{-1}\right) d\left(p w_{i} p^{\prime}\right) \\
& =\int_{\mathfrak{P} w_{i} \mathfrak{P}} \rho^{\vee}(p) \psi_{i}\left(w_{i}\right) \rho^{\vee}\left(p^{\prime}\right) \rho^{\vee}\left(p^{\prime}-1\right) \psi_{i}\left(w_{i}^{-1}\right) \rho^{\vee}\left(p^{-1}\right) d\left(p w_{i} p^{\prime}\right) \\
& =\int_{\mathfrak{P} w_{i} \mathfrak{P}} \rho^{\vee}(p) \psi_{i}\left(w_{i}\right) \psi_{i}\left(w_{i}^{-1}\right) \rho^{\vee}\left(p^{-1}\right) d\left(p w_{i} p^{\prime}\right) \\
& =\int_{\mathfrak{P} w_{i} \mathfrak{P}} \rho^{\vee}(p) \psi_{i}\left(w_{i}\right) \psi_{i}\left(w_{i}\right) \rho^{\vee}\left(p^{-1}\right) d\left(p w_{i} p^{\prime}\right) \\
& =\int_{\mathfrak{P} w_{i} \mathfrak{P}} \rho^{\vee}(p) A^{2} \rho^{\vee}\left(p^{-1}\right) d\left(p w_{i} p^{\prime}\right) \\
= & \int_{\mathfrak{P} w_{i} \mathfrak{P}} A^{2} \rho^{\vee}(p) \rho^{\vee}\left(p^{-1}\right) d\left(p w_{i} p^{\prime}\right) \\
= & A^{2} \operatorname{vol}\left(\mathfrak{P} w_{i} \mathfrak{P}\right)
\end{aligned}
$$




$$
=1_{W^{\vee} \operatorname{vol}\left(\mathfrak{P} w_{i} \mathfrak{P}\right)}
$$

So $\psi_{i}^{2}(1)=1_{W^{\vee}} \operatorname{vol}\left(\mathfrak{P} w_{i} \mathfrak{P}\right)$ for $i=0,1$. We already know that $\psi_{i}^{2}=a \psi_{i}+b$ where $a, b \in \mathbb{R}$ and for $i=0,1$. Now evaluating the expression $\psi_{i}^{2}=a \psi_{i}+b$ at 1 , we have $\psi_{i}^{2}(1)=a \psi_{i}(1)+b 1(1)$. We can see that $\psi_{i}(1)=0$ as support of $\psi_{i}$ is $\mathfrak{P} w_{i} \mathfrak{P}$ for $i=0,1$. We have seen before that $\psi_{i}^{2}(1)=1_{W^{\vee}} \operatorname{vol}\left(\mathfrak{P} w_{i} \mathfrak{P}\right)$ for $i=0,1$ and as $1 \in \mathfrak{P}, 1(1)=\rho^{\vee}(1)=1_{W^{\vee}}$. So $\psi_{i}^{2}(1)=a \psi_{i}(1)+b 1(1) \Longrightarrow$ $1_{W^{\vee}} \operatorname{vol}\left(\mathfrak{P} w_{i} \mathfrak{P}\right)=1_{W^{\vee}} b$ for $i=0,1$. Comparing coefficients of $1_{W^{\vee}}$ on both sides

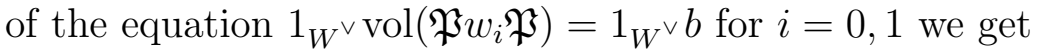

$$
b=\operatorname{vol}\left(\mathfrak{P} w_{i} \mathfrak{P}\right) .
$$

As $\phi_{i}=\lambda_{i} \psi_{i}$ for $i=0,1$, hence $\phi_{i}^{2}=\lambda_{i}^{2} \psi_{i}^{2}=\lambda_{i}^{2}\left(a \psi_{i}+b\right)=\left(\lambda_{i} a\right)\left(\lambda_{i} \psi_{i}\right)+$ $\lambda_{i}^{2} b=\left(\lambda_{i} a\right) \phi_{i}+\lambda_{i}^{2} b$ for $i=0,1$. But $\phi_{i}^{2}=\left(q^{n}-1\right) \phi_{i}+q^{n}$ for $i=0,1$. So $\phi_{i}^{2}=\left(\lambda_{i} a\right) \phi_{i}+\lambda_{i}^{2} b=\left(q^{n}-1\right) \phi_{i}+q^{n}$ for $i=0,1$. As $\phi_{i}$ and 1 are linearly independent, hence $\lambda_{i} a=\left(q^{n}-1\right)$ for $i=0,1$. Therefore $\lambda_{i}=\frac{q^{n}-1}{a}$ for $i=0,1$. As $a>0, a \in \mathbb{R}$, so $\lambda_{i}>0, \lambda_{i} \in \mathbb{R}$ for $i=0,1$. Similarly, as $\phi_{i}$ and 1 are linearly independent, hence $\lambda_{i}^{2} b=q^{n} \Longrightarrow \lambda_{i}^{2}=\frac{q^{n}}{b}$ for $i=0,1$.

Now $\mathfrak{P} w_{i} \mathfrak{P}=\underset{x \in \mathfrak{P} / \mathfrak{P} \cap w_{i} \mathfrak{P}}{\amalg} x w_{i} \mathfrak{P} \Longrightarrow \operatorname{vol}\left(\mathfrak{P} w_{i} \mathfrak{P}\right)=\left[\mathfrak{P} w_{i} \mathfrak{P}: \mathfrak{P}\right] \operatorname{vol} \mathfrak{P}=\left[\mathfrak{P} w_{i} \mathfrak{P}:\right.$ $\mathfrak{P}]=\left[\mathfrak{P}: \mathfrak{P} \cap^{w_{i}} \mathfrak{P}\right]$ for $i=0,1$. Hence $b=\operatorname{vol}\left(\mathfrak{P} w_{i} \mathfrak{P}\right)=\left[\mathfrak{P}: \mathfrak{P} \cap^{w_{i}} \mathfrak{P}\right]$ for $i=0,1$. Now as $\lambda_{0}^{2}=\lambda_{1}^{2}=\frac{q^{n}}{b} \Longrightarrow \lambda_{0}=\lambda_{1}=\frac{q^{n / 2}}{b^{1 / 2}}=\frac{q^{n / 2}}{\left[\mathfrak{P}: \mathfrak{P} \cap{ }^{w} 0 \mathfrak{P}^{1 / 2}\right.}$. Therefore

$$
\begin{aligned}
\phi_{0} \phi_{1} & =\left(\lambda_{0} \psi_{0}\right)\left(\lambda_{1} \psi_{1}\right) \\
& =\lambda_{0}^{2} \psi_{0} \psi_{1} \\
& =\frac{q^{n} \psi_{0} \psi_{1}}{\left[\mathfrak{P}: \mathfrak{P} \cap w_{0} \mathfrak{P}\right]} .
\end{aligned}
$$


We have seen before that, $\mathfrak{P}=K_{-, 1} \mathfrak{P}_{0} K_{+, 0}$ and $\mathfrak{P} \cap w_{0} \mathfrak{P}=K_{-, 1} \mathfrak{P}_{0} K_{+, 1}$. So

$$
\begin{aligned}
{\left[\mathfrak{P}: \mathfrak{P} \cap^{w_{0}} \mathfrak{P}\right] } & =\left|\frac{K_{+, 0}}{K_{+, 1}}\right| \\
& =\left|\left\{X \in \mathrm{M}_{n}\left(k_{E}\right) \mid X+{ }^{t} \bar{X}=0\right\}\right| \\
& =\left(q^{n}\right)\left(q^{2}\right)^{\frac{(n)(n-1)}{2}} \\
& =\left(q^{n}\right)\left(q^{n^{2}-n}\right) \\
& =q^{n^{2}} .
\end{aligned}
$$

Hence

$$
\begin{aligned}
\left(\phi_{0} \phi_{1}\right)(\zeta) & =\frac{q^{n}\left(\psi_{0} \psi_{1}\right)(\zeta)}{\left[\mathfrak{P}: \mathfrak{P} \cap \cap^{w_{0}} \mathfrak{P}\right]} \\
& =\frac{q^{n}\left(\psi_{0} \psi_{1}\right)(\zeta)}{q^{n^{2}}} \\
& =q^{n-n^{2}} 1_{W^{\vee}} .
\end{aligned}
$$

Recall $g_{i}=q^{-n / 2} \phi_{i}$ for $i=0,1$. We know that $\phi_{i}^{2}=\left(q^{n}-1\right) \phi_{i}+q^{n}$ for $i=0,1$. So for $i=0,1$ we have

$$
\begin{aligned}
g_{i}^{2} & =q^{-n} \phi_{i}^{2} \\
& =q^{-n}\left(\left(q^{n}-1\right) \phi_{i}+q^{n}\right) \\
& =\left(1-q^{-n}\right) \phi_{i}+1 \\
& =\left(1-q^{-n}\right) q^{n / 2} g_{i}+1 \\
& =\left(q^{n / 2}-q^{-n / 2}\right) g_{i}+1 .
\end{aligned}
$$


So $g_{0} g_{1}=\left(q^{-n / 2} \phi_{1}\right)\left(q^{-n / 2} \phi_{2}\right)=q^{-n} \phi_{1} \phi_{2} \Longrightarrow\left(g_{0} g_{1}\right)(\zeta)=q^{-n}\left(\phi_{1} \phi_{2}\right)(\zeta)=$ $q^{-n} q^{n-n^{2}} 1_{W^{\vee}}=q^{-n^{2}} 1_{W^{\vee}}$. From the earlier discussion in this section we have $T_{P}(\alpha)(\zeta)=\delta_{P}^{1 / 2}(\zeta) 1_{W^{\vee}}$. From section 4.1, we have $\delta_{P}(\zeta)=q^{-2 n^{2}}$. Hence $\delta_{P}^{1 / 2}(\zeta)=q^{-n^{2}}$. Therefore $\left(g_{0} g_{1}\right)(\zeta)=T_{P}(\alpha)(\zeta)$. So $\left(g_{0} g_{1}\right)(\zeta)=T_{P}(\alpha)(\zeta)$. We have $\operatorname{supp}\left(T_{P}(\alpha)\right)=\mathfrak{P} \zeta \mathfrak{P}$. As $\operatorname{supp}\left(g_{i}\right)=\mathfrak{P} w_{i} \mathfrak{P}$, Lemma 4.2 gives $\operatorname{supp}\left(g_{0} g_{1}\right)=$ $\mathfrak{P} \zeta \mathfrak{P}$. Therefore $g_{0} g_{1}=T_{P}(\alpha)$.

\subsubsection{Relation between $g_{0}, g_{1}$ and $T_{p}(\alpha)$ : ramified case}

We know that $\mathcal{H}(G, \rho)=\left\langle\phi_{0}, \phi_{1}\right\rangle$ where $\phi_{0}$ is supported on $\mathfrak{P} w_{0} \mathfrak{P}$ and $\phi_{1}$ is supported on $\mathfrak{P} w_{1} \mathfrak{P}$ respectively with $\phi_{i}^{2}=q^{n / 2}+\left(q^{n / 2}-1\right) \phi_{i}$ for $i=0,1$. In this section we show that $g_{0} * g_{1}=T_{P}(\alpha)$, where $g_{i}=q^{-n / 4} \phi_{i}$ for $i=0,1$.

Proposition 4.5. $g_{0} g_{1}=T_{P}(\alpha)$.

Proof. Let us choose $\psi_{i} \in \mathcal{H}(G, \rho)$ for $i=0,1$ such that $\operatorname{supp}\left(\psi_{i}\right)=\mathfrak{P} w_{i} \mathfrak{P}$ for $i=0,1$. So $\phi_{i}$ is a scalar multiple of $\psi_{i}$ for $i=0,1$. Hence $\phi_{i}=\lambda_{i} \psi_{i}$ where $\lambda_{i} \in \mathbb{C}^{\times}$for $i=0,1$. Let $\psi_{i}\left(w_{i}\right)=A_{i} \in \operatorname{Hom}_{\mathfrak{P} \cap{ }^{w} \mathfrak{i}}\left(w_{i} \rho^{\vee}, \rho^{\vee}\right)$ for $i=0,1$ and $W$ be the space of $\rho$. So $A_{i}^{2}=1_{W^{\vee}}$ for $i=0,1$. From section 5.1 on page 24 in [4], we can say that $A_{0}=A_{1}$. From Propn. 4.3, we have $\left(\psi_{0} * \psi_{1}\right)(\zeta)=$ $\psi_{0}\left(w_{0}\right) \psi_{1}\left(w_{1}\right)=A_{0} A_{1}=A_{0}^{2}=1_{W^{\vee}}$. Now let $\psi_{i}$ satisfies the quadratic relation given by $\psi_{i}^{2}=a_{i} \psi_{i}+b_{i}$ where $a_{i}, b_{i} \in \mathbb{R}$ for $i=0,1$. As $\psi_{i}^{2}=a_{i} \psi_{i}+b_{i} \Longrightarrow$ $\left(-\psi_{i}\right)^{2}=\left(-a_{i}\right)\left(-\psi_{i}\right)+b_{i}$, so $a_{i}$ can be arranged such that $a_{i}>0$ for $i=0,1$. We can see that $1 \in \mathcal{H}(G, \rho)$ is defined as below:

$$
1(x)= \begin{cases}0, & \text { if } x \notin \mathfrak{P} \\ \rho^{\vee}(x) & \text { if } x \in \mathfrak{P} .\end{cases}
$$


Let us consider $\psi_{0}^{2}(1)=\int_{G} \psi_{0}(y) \psi_{0}\left(y^{-1}\right) d y$. Now let $y=p w_{0} p^{\prime}$ where $p, p^{\prime} \in$ $\mathfrak{P}$. So we have

$$
\begin{aligned}
\psi_{0}^{2}(1) & =\int_{\mathfrak{P} w_{0} \mathfrak{P}} \psi_{0}\left(p w_{0} p^{\prime}\right) \psi_{0}\left(p^{\prime}-1 w_{0}^{-1} p^{-1}\right) d\left(p w_{0} p^{\prime}\right) \\
& =\int_{\mathfrak{P} w_{0} \mathfrak{P}} \rho^{\vee}(p) \psi_{0}\left(w_{0}\right) \rho^{\vee}\left(p^{\prime}\right) \rho^{\vee}\left(p^{\prime}-1\right) \psi_{0}\left(w_{0}^{-1}\right) \rho^{\vee}\left(p^{-1}\right) d\left(p w_{0} p^{\prime}\right) \\
& =\int_{\mathfrak{P} w_{0} \mathfrak{P}} \rho^{\vee}(p) \psi_{0}\left(w_{0}\right) \psi_{0}\left(w_{0}^{-1}\right) \rho^{\vee}\left(p^{-1}\right) d\left(p w_{0} p^{\prime}\right) \\
& =\int_{\mathfrak{P} w_{0} \mathfrak{P}} \rho^{\vee}(p) \psi_{0}\left(w_{0}\right) \psi_{0}\left(w_{0}\right) \rho^{\vee}\left(p^{-1}\right) d\left(p w_{0} p^{\prime}\right) \\
& =\int_{\mathfrak{P} w_{0} \mathfrak{P}} \rho^{\vee}(p) A_{0}^{2} \rho^{\vee}\left(p^{-1}\right) d\left(p w_{0} p^{\prime}\right) \\
& =\int_{\mathfrak{P} w_{0} \mathfrak{P}} A_{0}^{2} \rho^{\vee}(p) \rho^{\vee}\left(p^{-1}\right) d\left(p w_{0} p^{\prime}\right) \\
= & A_{0}^{2} \operatorname{vol}\left(\mathfrak{P} w_{0} \mathfrak{P}\right) \\
= & 1_{W^{\vee} \operatorname{vol}\left(\mathfrak{P} w_{0} \mathfrak{P}\right) .}
\end{aligned}
$$

So $\psi_{0}^{2}(1)=1_{W} \vee \operatorname{vol}\left(\mathfrak{P} w_{0} \mathfrak{P}\right)$. We already know that $\psi_{0}^{2}=a_{0} \psi_{0}+b_{0}$ where $a_{0}, b_{0} \in \mathbb{R}$. Now evaluating the expression $\psi_{0}^{2}=a_{0} \psi_{0}+b_{0}$ at 1 , we have $\psi_{0}^{2}(1)=$ $a_{0} \psi_{0}(1)+b_{0} 1(1)$. We can see that $\psi_{0}(1)=0$ as support of $\psi_{0}$ is $\mathfrak{P} w_{0} \mathfrak{P}$. We have

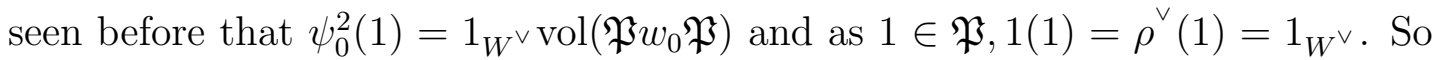
$\psi_{0}^{2}(1)=a_{0} \psi_{i}(1)+b_{0} 1(1) \Longrightarrow 1_{W^{\vee}} \operatorname{vol}\left(\mathfrak{P} w_{0} \mathfrak{P}\right)=1_{W^{\vee}} b_{0}$. Comparing coefficients of $1_{W^{\vee}}$ on both sides of the equation $1_{W^{\vee}} b_{0}=1_{W^{\vee}} \operatorname{vol}\left(\mathfrak{P} w_{0} \mathfrak{P}\right)$ we get

$$
b_{0}=\operatorname{vol}\left(\mathfrak{P} w_{0} \mathfrak{P}\right) .
$$

As $\phi_{0}=\lambda_{0} \psi_{0}$, hence $\phi_{0}^{2}=\lambda_{0}^{2} \psi_{0}^{2}=\lambda_{0}^{2}\left(a_{0} \psi_{0}+b_{0}\right)=\left(\lambda_{0} a_{0}\right)\left(\lambda_{0} \psi_{0}\right)+\lambda_{0}^{2} b_{0}=$ $\left(\lambda_{0} a_{0}\right) \phi_{0}+\lambda_{0}^{2} b_{0}$. But $\phi_{0}^{2}=\left(q^{n / 2}-1\right) \phi_{0}+q^{n / 2}$. So $\phi_{0}^{2}=\left(\lambda_{0} a_{0}\right) \phi_{0}+\lambda_{0}^{2} b_{0}=$ $\left(q^{n / 2}-1\right) \phi_{0}+q^{n / 2}$. As $\phi_{0}$ and 1 are linearly independent, hence $\lambda_{0} a_{0}=\left(q^{n / 2}-1\right)$. 
Therefore $\lambda_{0}=\frac{q^{n / 2}-1}{a_{0}}$. As $a_{0}>0, a_{0} \in \mathbb{R}$, so $\lambda_{0}>0, \lambda_{0} \in \mathbb{R}$. Similarly, as $\phi_{0}$ and 1 are linearly independent, hence $\lambda_{0}^{2} b=q^{n / 2} \Longrightarrow \lambda_{0}^{2}=\frac{q^{n / 2}}{b_{0}}$.

Now $\mathfrak{P} w_{0} \mathfrak{P}=\underset{x \in \mathfrak{P} / \mathfrak{P} \cap w_{0} \mathfrak{P}}{\amalg} x w_{0} \mathfrak{P} \Longrightarrow \operatorname{vol}\left(\mathfrak{P} w_{0} \mathfrak{P}\right)=\left[\mathfrak{P} w_{0} \mathfrak{P}: \mathfrak{P}\right] \operatorname{vol} \mathfrak{P}=\left[\mathfrak{P} w_{0} \mathfrak{P}:\right.$ $\mathfrak{P}]=\left[\mathfrak{P}: \mathfrak{P} \cap \cap^{w_{0}} \mathfrak{P}\right]$. Hence $b_{0}=\operatorname{vol}\left(\mathfrak{P} w_{0} \mathfrak{P}\right)=\left[\mathfrak{P}: \mathfrak{P} \cap \cap^{w_{0}} \mathfrak{P}\right]$. Now as $\lambda_{0}^{2}=\frac{q^{n / 2}}{b_{0}} \Longrightarrow \lambda_{0}=\frac{q^{n / 4}}{b_{0}^{1 / 2}}=\frac{q^{n / 4}}{\left[\mathfrak{P}: \mathfrak{P} \cap \cap^{w_{0}} \mathfrak{P}\right]^{1 / 2}}$.

We have seen before that, $\mathfrak{P}=K_{-, 1} \mathfrak{P}_{0} K_{+, 0}$ and $\mathfrak{P} \cap \cap^{w_{0}} \mathfrak{P}=K_{-, 1} \mathfrak{P}_{0} K_{+, 1}$. So

$$
\begin{aligned}
{\left[\mathfrak{P}: \mathfrak{P} \cap{ }^{w_{0}} \mathfrak{P}\right] } & =\left|\frac{K_{+, 0}}{K_{+, 1}}\right| \\
& =\left|\left\{X \in \mathrm{M}_{n}\left(k_{E}\right) \mid X+{ }^{t} \bar{X}=0\right\}\right| \\
& =q^{\frac{(n)(n-1)}{2}} \\
& =q^{\frac{n^{2}-n}{2}} .
\end{aligned}
$$

So

$$
\lambda_{0}=\frac{q^{n / 4}}{\left[\mathfrak{P}: \mathfrak{P} \cap^{w_{0}} \mathfrak{P}\right]^{1 / 2}}=\frac{q^{n / 4}}{q^{\frac{n^{2}-n}{4}}} .
$$

Let us consider $\psi_{1}^{2}(1)=\int_{G} \psi_{1}(y) \psi_{1}\left(y^{-1}\right) d y$. Now let $y=p w_{1} p^{\prime}$ where $p, p^{\prime} \in$ $\mathfrak{P}$. So we have

$$
\begin{aligned}
\psi_{1}^{2}(1) & =\int_{\mathfrak{P} w_{1} \mathfrak{P}} \psi_{1}\left(p w_{1} p^{\prime}\right) \psi_{1}\left(p^{\prime}-1 w_{1}^{-1} p^{-1}\right) d\left(p w_{1} p^{\prime}\right) \\
& =\int_{\mathfrak{P} w_{1} \mathfrak{P}} \rho^{\vee}(p) \psi_{1}\left(w_{1}\right) \rho^{\vee}\left(p^{\prime}\right) \rho^{\vee}\left(p^{\prime}-1\right) \psi_{1}\left(w_{1}^{-1}\right) \rho^{\vee}\left(p^{-1}\right) d\left(p w_{1} p^{\prime}\right) \\
& =\int_{\mathfrak{P} w_{1} \mathfrak{P}} \rho^{\vee}(p) \psi_{1}\left(w_{1}\right) \psi_{1}\left(w_{1}^{-1}\right) \rho^{\vee}\left(p^{-1}\right) d\left(p w_{1} p^{\prime}\right) \\
& =\int_{\mathfrak{P} w_{1} \mathfrak{P}} \rho^{\vee}(p) \psi_{1}\left(w_{1}\right) \psi_{1}\left(-w_{1}\right) \rho^{\vee}\left(p^{-1}\right) d\left(p w_{1} p^{\prime}\right)
\end{aligned}
$$




$$
\begin{aligned}
& =\int_{\mathfrak{P} w_{1} \mathfrak{P}} \rho^{\vee}(p) \psi_{1}\left(w_{1}\right) \rho^{\vee}(-1) \psi_{1}\left(w_{1}\right) \rho^{\vee}\left(p^{-1}\right) d\left(p w_{1} p^{\prime}\right) \\
& =\rho^{\vee}(-1) \int_{\mathfrak{P} w_{1} \mathfrak{P}} A_{1}^{2} \rho^{\vee}(p) \rho^{\vee}\left(p^{-1}\right) d\left(p w_{1} p^{\prime}\right) \\
& =\rho^{\vee}(-1) A_{1}^{2} \operatorname{vol}\left(\mathfrak{P} w_{1} \mathfrak{P}\right) \\
& =\rho^{\vee}(-1) 1_{W^{\vee} \operatorname{vol}\left(\mathfrak{P} w_{1} \mathfrak{P}\right) .}
\end{aligned}
$$

So $\psi_{1}^{2}(1)=1_{W^{\vee}} \operatorname{vol}\left(\mathfrak{P} w_{1} \mathfrak{P}\right)$. We already know that $\psi_{1}^{2}=a_{1} \psi_{1}+b_{1}$ where $a_{1}, b_{1} \in \mathbb{R}$. Now evaluating the expression $\psi_{1}^{2}=a_{1} \psi_{1}+b_{1}$ at 1 , we have $\psi_{1}^{2}(1)=$ $a_{1} \psi_{1}(1)+b_{1} 1(1)$. We can see that $\psi_{1}(1)=0$ as support of $\psi_{1}$ is $\mathfrak{P} w_{1} \mathfrak{P}$. We have seen before that $\psi_{1}^{2}(1)=1_{W^{\vee}} \operatorname{vol}\left(\mathfrak{P} w_{1} \mathfrak{P}\right)$ and as $1 \in \mathfrak{P}, 1(1)=\rho^{\vee}(1)=1_{W^{\vee}}$.

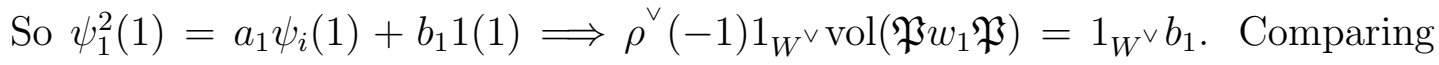
coefficients of $1_{W^{\vee}}$ on both sides of the equation $1_{W^{\vee}} b_{1}=1_{W^{\vee}} \rho^{\vee}(-1) \operatorname{vol}\left(\mathfrak{P} w_{1} \mathfrak{P}\right)$ we get

$$
b_{1}=\rho^{\vee}(-1) \operatorname{vol}\left(\mathfrak{P} w_{1} \mathfrak{P}\right) .
$$

As $\phi_{1}=\lambda_{1} \psi_{1}$, hence $\phi_{1}^{2}=\lambda_{1}^{2} \psi_{1}^{2}=\lambda_{1}^{2}\left(a_{1} \psi_{1}+b_{1}\right)=\left(\lambda_{1} a_{1}\right)\left(\lambda_{1} \psi_{1}\right)+\lambda_{1}^{2} b_{1}=$ $\left(\lambda_{0} a_{1}\right) \phi_{1}+\lambda_{1}^{2} b_{1}$. But $\phi_{1}^{2}=\left(q^{n / 2}-1\right) \phi_{1}+q^{n / 2}$. So $\phi_{1}^{2}=\left(\lambda_{1} a_{1}\right) \phi_{1}+\lambda_{1}^{2} b_{1}=$ $\left(q^{n / 2}-1\right) \phi_{1}+q^{n / 2}$. As $\phi_{1}$ and 1 are linearly independent, hence $\lambda_{1} a_{1}=\left(q^{n / 2}-1\right)$. Therefore $\lambda_{1}=\frac{q^{n / 2}-1}{a_{1}}$. As $a_{1}>0, a_{1} \in \mathbb{R}$, so $\lambda_{1}>0, \lambda_{1} \in \mathbb{R}$. Similarly, as $\phi_{1}$ and 1 are linearly independent, hence $\lambda_{1}^{2} b=q^{n / 2} \Longrightarrow \lambda_{1}^{2}=\frac{q^{n / 2}}{b_{1}}$.

Now $\mathfrak{P} w_{1} \mathfrak{P}=\underset{x \in \mathfrak{P} / \mathfrak{P} \cap w_{1} \mathfrak{P}}{\amalg} x w_{1} \mathfrak{P} \Longrightarrow \operatorname{vol}\left(\mathfrak{P} w_{1} \mathfrak{P}\right)=\left[\mathfrak{P} w_{1} \mathfrak{P}: \mathfrak{P}\right] \operatorname{vol} \mathfrak{P}=\left[\mathfrak{P} w_{1} \mathfrak{P}:\right.$ $\mathfrak{P}]=\left[\mathfrak{P}: \mathfrak{P} \cap^{w_{1}} \mathfrak{P}\right]$. Hence $b_{1}=\operatorname{vol}\left(\mathfrak{P} w_{1} \mathfrak{P}\right)=\left[\mathfrak{P}: \mathfrak{P} \cap^{w_{1}} \mathfrak{P}\right]$. Now as $\lambda_{1}^{2}=\frac{q^{n / 2}}{b_{1}} \Longrightarrow \lambda_{1}=\frac{q^{n / 4}}{b_{1}^{1 / 2}}=\frac{q^{n / 4}}{\left[\mathfrak{P}: \mathfrak{P} \cap^{w_{1}} \mathfrak{P}\right]^{1 / 2}}$.

We have seen before that $\mathfrak{P}=K_{-, 1} \mathfrak{P}_{0} K_{+, 0},{ }^{w_{1}} \mathfrak{P}=K_{-, 2} \mathfrak{P}_{0} K_{+,-1}$. So $\mathfrak{P} \cap^{w_{1}}$ $\mathfrak{P}=K_{-, 2} \mathfrak{P}_{0} K_{+, 0}$. Hence 


$$
\begin{aligned}
{\left[\mathfrak{P}: \mathfrak{P} \cap{ }^{w_{1}} \mathfrak{P}\right] } & =\left|\frac{K_{-, 1}}{K_{-, 2}}\right| \\
& =\left|\left\{X \in \mathrm{M}_{n}\left(k_{E}\right) \mid X={ }^{t} \bar{X}\right\}\right| \\
& =q^{\frac{(n)(n+1)}{2}} \\
& =q^{\frac{n^{2}+n}{2}} .
\end{aligned}
$$

So

$$
\lambda_{1}=\frac{q^{n / 4}}{\left[\mathfrak{P}: \mathfrak{P} \cap^{w_{1}} \mathfrak{P}\right]^{1 / 2}}=\frac{q^{n / 4}}{q^{\frac{n^{2}+n}{4}}(\rho(-1))^{1 / 2}} .
$$

Hence

$$
\begin{aligned}
\left(\phi_{0} \phi_{1}\right)(\zeta) & =\left(\lambda_{0} \psi_{0}\right)\left(\lambda_{1} \psi_{1}\right)(\zeta) \\
& =\left(\lambda_{0} \lambda_{1}\right)\left(\psi_{0} \psi_{1}\right)(\zeta) \\
& =\frac{q^{n / 4}}{q^{\frac{n^{2}-n}{4}}} \frac{q^{n / 4}}{q^{\frac{n^{2}+n}{4}}(\rho(-1))^{1 / 2}} 1_{W}^{\vee} \\
& =\frac{q^{\frac{n-n^{2}}{2}}}{(\rho(-1))_{W}^{1 / 2}} \\
& =\frac{q^{\frac{n-n^{2}}{2}}}{(\rho(-1))_{W}^{1 / 2}} .
\end{aligned}
$$

As $-1 \in Z(\mathfrak{P})$ and $\rho^{\vee}$ is a representation of $\mathfrak{P}$, so $\rho^{\vee}(-1)=\omega_{\rho^{\vee}}(-1)$ where $\omega_{\rho^{\vee}}$ is the central character of $\mathfrak{P}$. Now $1=\omega_{\rho^{\vee}}(1)=\left(\omega_{\rho^{\vee}}(-1)\right)^{2}$, so $\rho^{\vee}(-1)=$ $\omega_{\rho} \vee(-1)= \pm 1$. We have seen before that $\lambda_{1}=\frac{q^{n / 2}-1}{a_{1}}$ and $a_{1} \in \mathbb{R}, a_{1}>0$, so $\lambda_{1}>0$. But we know that $\lambda_{1}=\frac{q^{n / 4}}{\left[\mathfrak{P}: \mathfrak{P}^{w_{1}} \mathfrak{P}^{1 / 2}\right]^{1 / 2}}=\frac{q^{n / 4}}{q^{\frac{n^{2}+n}{4}}(\rho(-1))^{1 / 2}}$, hence $\rho^{\vee}(-1)=1$.

Recall $g_{i}=q^{-n / 4} \phi_{i}$ for $i=0,1$. We know that $\phi_{i}^{2}=\left(q^{n / 2}-1\right) \phi_{i}+q^{n / 2}$ for $i=0,1$. So for $i=0,1$ we have 


$$
\begin{aligned}
g_{i}^{2} & =q^{-n / 2} \phi_{i}^{2} \\
& =q^{-n / 2}\left(\left(q^{n / 2}-1\right) \phi_{i}+q^{n / 2}\right) \\
& =\left(1-q^{-n / 2}\right) \phi_{i}+1 \\
& =\left(1-q^{-n / 2}\right) q^{n / 4} g_{i}+1 \\
& =\left(q^{n / 4}-q^{-n / 4}\right) g_{i}+1
\end{aligned}
$$

So $g_{0} g_{1}=\left(q^{-n / 4} \phi_{1}\right)\left(q^{-n / 4} \phi_{2}\right)=q^{-n / 2} \phi_{1} \phi_{2} \Longrightarrow\left(g_{0} g_{1}\right)(\zeta)=q^{-n / 2}\left(\phi_{0} \phi_{1}\right)(\zeta)=$ $q^{-n / 2} \frac{q^{\frac{n-n^{2}}{2}}}{(\rho(-1))_{W}^{1 / 2}}=q^{\frac{-n^{2}}{2}} 1_{W}^{\vee}$. From the earlier discussion in this section we have $T_{P}(\alpha)(\zeta)=\delta_{P}^{1 / 2}(\zeta) 1_{W^{\vee}}$. From section 4.1, we have $\delta_{P}(\zeta)=q^{-n^{2}}$. Hence $\delta_{P}^{1 / 2}(\zeta)=$ $q^{-n^{2} / 2}$. Therefore $\left(g_{0} g_{1}\right)(\zeta)=T_{P}(\alpha)(\zeta)$. So $\left(g_{0} g_{1}\right)(\zeta)=T_{P}(\alpha)(\zeta)$. We have $\operatorname{supp}\left(T_{P}(\alpha)\right)=\mathfrak{P} \zeta \mathfrak{P}$. As $\operatorname{supp}\left(g_{i}\right)=\mathfrak{P} w_{i} \mathfrak{P}$, Lemma 4.2 gives $\operatorname{supp}\left(g_{0} g_{1}\right)=\mathfrak{P} \zeta \mathfrak{P}$. Therefore $g_{0} g_{1}=T_{P}(\alpha)$.

\subsection{Calculation of $m_{L}(\pi \nu)$}

Note $\pi \nu$ lies in $\mathfrak{R}^{[L, \pi]_{L}}(L)$. Recall $m_{L}$ is an equivalence of categories. As $\pi \nu$ is an irreducible representation of $L$, it follows that $m_{L}(\pi \nu)$ is a simple $\mathcal{H}\left(L, \rho_{0}\right)$ module. In this section, we identify the simple $\mathcal{H}\left(L, \rho_{0}\right)$-module corresponding to $m_{L}(\pi \nu)$. Calculating $m_{L}(\pi \nu)$ will be useful in answering the question in next section.

From section 2.4 , we know that $\pi=\operatorname{Ind} \frac{L}{\widetilde{\mathfrak{P}_{0}}} \widetilde{\rho_{0}}$, where $\widetilde{\mathfrak{P}_{0}}=\langle\zeta\rangle \mathfrak{P}_{0}, \widetilde{\rho_{0}}\left(\zeta^{k} j\right)=$ $\rho_{0}(j)$ for $j \in \mathfrak{P}_{0}, k \in \mathbb{Z}$. Let us recall that $\nu$ is unramified character of $L$ from section 2.3. Let $V$ be space of $\pi \nu$ and $W$ be space of $\rho_{0}$. Recall $m_{L}(\pi \nu)=$ $\operatorname{Hom}_{\mathfrak{P}_{0}}\left(\rho_{0}, \pi \nu\right)$. Let $f \in \operatorname{Hom}_{\mathfrak{P}_{0}}\left(\rho_{0}, \pi \nu\right)$. As $\mathfrak{P}_{0}$ is a compact open subgroup of $L$ 
and $\nu$ is an unramified character of $L$, so $\nu(j)=1$ for $j \in \mathfrak{P}_{0}$. We already know that $\alpha \in \mathcal{H}\left(L, \rho_{0}\right)$ with support of $\alpha$ being $\mathfrak{P}_{0} \zeta$ and $\alpha(\zeta)=1_{W^{\vee}}$. Let $w \in W$ and we have seen in section 4.2 that the way $\mathcal{H}\left(L, \rho_{0}\right)$ acts on $\operatorname{Hom}_{\mathfrak{P}_{0}}\left(\rho_{0}, \pi \nu\right)$ is given by:

$$
\begin{aligned}
(\alpha . f)(w) & =\int_{L}(\pi \nu)(l) f\left(\alpha^{\vee}\left(l^{-1}\right) w\right) d l \\
& =\int_{L}(\pi \nu)(l) f\left((\alpha(l))^{\vee} w\right) d l \\
& =\int_{\mathfrak{P}_{0}}(\pi \nu)(p \zeta) f\left((\alpha(p \zeta))^{\vee} w\right) d p \\
& =\int_{\mathfrak{P}_{0}}(\pi \nu)(p \zeta) f\left(\left(\rho_{0}^{\vee}(p) \alpha(\zeta)\right)^{\vee} w\right) d p \\
& =\int_{\mathfrak{P}_{0}}(\pi \nu)(p \zeta) f\left(\left(\rho_{0}^{\vee}(p) 1_{W^{\vee}}\right)^{\vee} w\right) d p \\
& =\int_{\mathfrak{P}_{0}}(\pi \nu)(p \zeta) f\left(\left(\rho_{0}^{\vee}(p)\right)^{\vee} w\right) d p \\
& =\int_{\mathfrak{P}_{0}} \pi(p \zeta) \nu(p \zeta) f\left(\left(\rho_{0}^{\vee}(p)\right)^{\vee} w\right) d p \\
& =\int_{\mathfrak{P}_{0}} \pi(p \zeta) \nu(\zeta) f\left(\left(\rho_{0}^{\vee}(p)\right)^{\vee} w\right) d p .
\end{aligned}
$$

Now $\langle\rangle:, W \times W^{\vee} \longrightarrow \mathbb{C}$ is given by: $\left\langle w, \rho_{0}^{\vee}(p) w^{\vee}\right\rangle=\left\langle\rho_{0}\left(p^{-1}\right) w, w^{\vee}\right\rangle$ for $p \in \mathfrak{P}_{0}, w \in W$. So we have $\left(\rho_{0}^{\vee}(p)\right)^{\vee}=\rho_{0}\left(p^{-1}\right)$ for $p \in \mathfrak{P}_{0}$. Hence

$$
(\alpha . f)(w)=\int_{\mathfrak{P}_{0}} \pi(p \zeta) \nu(\zeta) f\left(\rho_{0}\left(p^{-1}\right) w\right) d p
$$

As $f \in \operatorname{Hom}_{\mathfrak{P}_{0}}\left(\rho_{0}, \pi \nu\right)$, so $(\pi \nu)(p) f(w)=f\left(\rho_{0}(p) w\right)$ for $p \in \mathfrak{P}_{0}, w \in W$. 
Hence

$$
\begin{aligned}
(\alpha . f)(w) & =\nu(\zeta) \int_{\mathfrak{P}_{0}} \pi(p \zeta)(\pi \nu)\left(p^{-1}\right) f(w) d p \\
& =\nu(\zeta) \int_{\mathfrak{P}_{0}} \pi(p \zeta) \pi\left(p^{-1}\right) \nu\left(p^{-1}\right) f(w) d p \\
& =\nu(\zeta) \int_{\mathfrak{P}_{0}} \pi(p \zeta) \pi\left(p^{-1}\right) f(w) d p .
\end{aligned}
$$

Now as $\pi=\operatorname{Ind} d_{\mathfrak{P}_{0}}^{L} \widetilde{\rho}_{0}$ and $\widetilde{\mathfrak{P}_{0}}=\langle\zeta\rangle \mathfrak{P}_{0}, \widetilde{\rho}_{0}\left(\zeta^{k} j\right)=\rho_{0}(j)$ for $j \in \mathfrak{P}_{0}, k \in \mathbb{Z}$, so $\pi(p \zeta)=\pi(p) \widetilde{\rho_{0}}(\zeta)=\pi(p) \rho_{0}(1)=\pi(p) 1_{W^{\vee}}$. Therefore

$$
\begin{aligned}
(\alpha . f)(w) & =\nu(\zeta) \int_{\mathfrak{P}_{0}} \pi(p) \pi\left(p^{-1}\right) f(w) d p \\
& =\nu(\zeta) f(w) \operatorname{Vol}\left(\mathfrak{P}_{0}\right) \\
& =\nu(\zeta) f(w)
\end{aligned}
$$

So $(\alpha . f)(w)=\nu(\zeta) f(w)$ for $w \in W$. So $\alpha$ acts on $f$ by multiplication by $\nu(\zeta)$. Recall for $\lambda \in \mathbb{C}^{\times}$, we write $\mathbb{C}_{\lambda}$ for the $\mathcal{H}\left(L, \rho_{0}\right)$-module with underlying abelian group $\mathbb{C}$ such that $\alpha . z=\lambda z$ for $z \in \mathbb{C}_{\lambda}$. Therefore $m_{L}(\pi \nu) \cong \mathbb{C}_{\nu(\zeta)}$.

\subsection{Answering the question}

Recall the following commutative diagram which we described earlier.

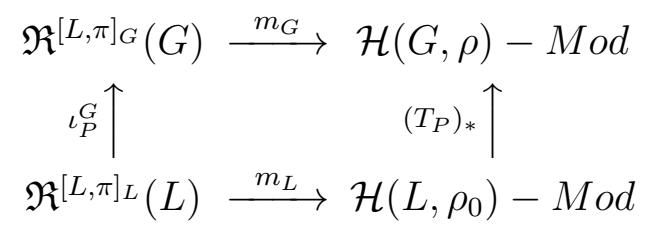


Observe that $\pi \nu$ lies in $\mathfrak{R}^{[L, \pi]_{L}}(L)$. From the above commutative diagram, it follows that $\iota_{P}^{G}(\pi \nu)$ lies in $\mathfrak{R}^{[L, \pi]_{G}}(G)$ and $m_{G}\left(\iota_{P}^{G}(\pi \nu)\right)$ is an $\mathcal{H}(G, \rho)$-module. Recall $m_{L}(\pi \nu) \cong \mathbb{C}_{\nu(\zeta)}$ as $\mathcal{H}\left(L, \rho_{0}\right)$-modules. From the above commutative diagram, we have $m_{G}\left(\iota_{P}^{G}(\pi \nu)\right) \cong\left(T_{P}\right)_{*}\left(\mathbb{C}_{\nu(\zeta)}\right)$ as $\mathcal{H}(G, \rho)$-modules. Thus to determine the unramified characters $\nu$ for which $\iota_{P}^{G}(\pi \nu)$ is irreducible, we have to understand when $\left(T_{P}\right)_{*}\left(\mathbb{C}_{\nu(\zeta)}\right)$ is a simple $\mathcal{H}(G, \rho)$-module.

Using notation on page 438 in [5], we have $\gamma_{1}=\gamma_{2}=q^{n / 2}$ for unramified case when $n$ is odd and $\gamma_{1}=\gamma_{2}=q^{n / 4}$ for ramified case when $n$ is even. As in Propn. 1.6 of [5], let $\Gamma=\left\{\gamma_{1} \gamma_{2},-\gamma_{1} \gamma_{2}^{-1},-\gamma_{1}^{-1} \gamma_{2},\left(\gamma_{1} \gamma_{2}\right)^{-1}\right\}$. So by Propn. 1.6 in [5], $\left(T_{P}\right)_{*}\left(\mathbb{C}_{\nu(\zeta)}\right)$ is a simple $\mathcal{H}(G, \rho)$-module $\Longleftrightarrow \nu(\zeta) \notin \Gamma$. Recall $\pi=\operatorname{Ind} d_{Z(L) \mathfrak{P}_{0}}^{L} \widetilde{\rho_{0}}$ where $\widetilde{\rho_{0}}\left(\zeta^{k} j\right)=\rho_{0}(j)$ for $j \in \mathfrak{P}_{0}, k \in \mathbb{Z}$ and $\rho_{0}=\tau_{\theta}$ for some regular character $\theta$ of $l^{\times}$with $\left[l: k_{E}\right]=n$. Hence we can conclude that $\iota_{P}^{G}(\pi \nu)$ is irreducible for the unramified case when $n$ is odd $\Longleftrightarrow \nu(\zeta) \notin\left\{q^{n}, q^{-n},-1\right\}$, $\theta^{q^{n+1}}=\theta^{-q}$ and $\iota_{P}^{G}(\pi \nu)$ is irreducible for the ramified case when $n$ is even $\Longleftrightarrow \nu(\zeta) \notin\left\{q^{n / 2}, q^{-n / 2},-1\right\}, \theta^{q^{n / 2}}=\theta^{-1}$.

Recall that in the unramified case when $n$ is even or in the ramified case when $n$ is odd we have $N_{G}\left(\rho_{0}\right)=Z(L) \mathfrak{P}_{0}$. Thus $\mathfrak{I}_{G}(\rho)=\mathfrak{P}\left(Z(L) \mathfrak{P}_{0}\right) \mathfrak{P}=\mathfrak{P} Z(L) \mathfrak{P}$.

From Corollary 6.5 in [6] which states that if $\mathfrak{I}_{G}(\rho) \subseteq \mathfrak{P} L \mathfrak{P}$ then

$$
T_{P}: \mathcal{H}\left(L, \rho_{0}\right) \longrightarrow \mathcal{H}(G, \rho)
$$

is an isomorphism of $\mathbb{C}$-algebras. As we have $\mathfrak{I}_{G}(\rho)=\mathfrak{P} Z(L) \mathfrak{P}$ in the unramified case when $n$ is even or in the ramified case when $n$ is odd, so $\mathcal{H}\left(L, \rho_{0}\right) \cong \mathcal{H}(G, \rho)$ as $\mathbb{C}$-algebras. So from the commutative diagram on page 80 , we can conclude that $\iota_{P}^{G}(\pi \nu)$ is irreducible for any unramified character $\nu$ of $L$. So we conclude with the following theorem. 
Theorem 4.6. Let $G=\mathrm{U}(n, n)$. Let $P$ be the Siegel parabolic subgroup of $G$ and $L$ be the Siegel Levi component of P. Let $\pi=\operatorname{Ind}_{Z(L) \mathfrak{P}_{0}}^{L}{\widetilde{\rho_{0}}}_{\text {be }}$ a smooth irreducible supercuspidal depth zero representation of $L \cong \mathrm{GL}_{n}(E)$ where ${\widetilde{\rho_{0}}}_{(}\left(\zeta^{k} j\right)=\rho_{0}(j)$ for $j \in \mathfrak{P}_{0}, k \in \mathbb{Z}$ and $\rho_{0}=\tau_{\theta}$ for some regular character $\theta$ of $l^{\times}$with $\left[l: k_{E}\right]=n$. Consider the family $\iota_{P}^{G}(\pi \nu)$ for $\nu \in \mathrm{X}_{n r}(L)$.

1. For $E / F$ is unramified, $\iota_{P}^{G}(\pi \nu)$ is reducible $\Longleftrightarrow n$ is odd, $\theta^{q^{n+1}}=\theta^{-q}$ and $\nu(\zeta) \in\left\{q^{n}, q^{-n},-1\right\}$.

2. For $E / F$ is ramified, $\iota_{P}^{G}(\pi \nu)$ is reducible $\Longleftrightarrow n$ is even, $\theta^{q^{n / 2}}=\theta^{-1}$ and $\nu(\zeta) \in\left\{q^{n / 2}, q^{-n / 2},-1\right\}$ 


\section{Bibliography}

[1] M. F. Atiyah and I. G. Macdonald. Introduction to commutative algebra. Addison-Wesley Series in Mathematics. Westview Press, Boulder, CO, economy edition, 2016.

[2] Colin J. Bushnell and Philip C. Kutzko. Smooth representations of reductive p-adic groups: structure theory via types. Proc. London Math. Soc. (3), 77(3):582-634, 1998.

[3] David S. Dummit and Richard M. Foote. Abstract algebra. John Wiley \& Sons, Inc., Hoboken, NJ, third edition, 2004.

[4] Philip Kutzko and Lawrence Morris. Level zero Hecke algebras and parabolic induction: the Siegel case for split classical groups. Int. Math. Res. Not., pages Art. ID 97957, 40, 2006.

[5] Philip Kutzko and Lawrence Morris. Explicit Plancherel theorems for $\mathcal{H}\left(q_{1}, q_{2}\right)$ and $\mathbb{S L}_{2}(F)$. Pure Appl. Math. Q., 5(1):435-467, 2009.

[6] Philip C. Kutzko. Smooth representations of reductive p-adic groups: an introduction to the theory of types. In Geometry and representation theory of real and p-adic groups (Córdoba, 1995), volume 158 of Progr. Math., pages 175-196. Birkhäuser Boston, Boston, MA, 1998. 
[7] Thomas Laebel Madsen. Some types and covers for quaternionic hermitian groups. PhD thesis, University of Oklahoma, 2014.

[8] Lawrence Morris. Tamely ramified intertwining algebras. Invent. Math., 114(1):1-54, 1993.

[9] Allen Moy and Gopal Prasad. Jacquet functors and unrefined minimal $K$ types. Comment. Math. Helv., 71(1):98-121, 1996. 